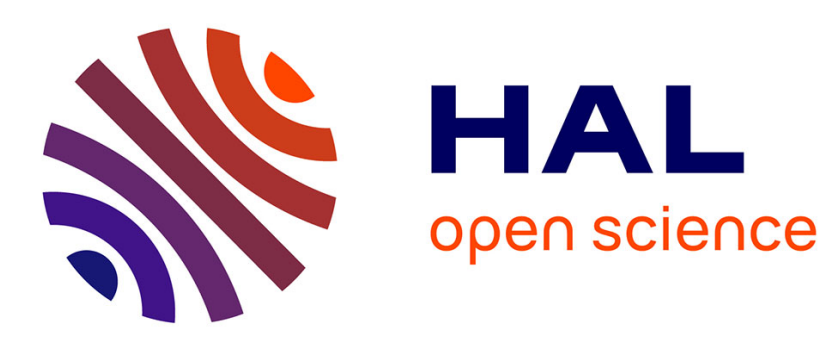

\title{
Application of Molecular Dynamics DL_POLY Code to Interfaces of Inorganic Materials
}

Paul Martin, Steve Parker, Dino Spagnoli, Arnaud Marmier, Dean Sayle, Graeme Watson

\section{- To cite this version:}

Paul Martin, Steve Parker, Dino Spagnoli, Arnaud Marmier, Dean Sayle, et al.. Application of Molecular Dynamics DL_POLY Code to Interfaces of Inorganic Materials. Molecular Simulation, 2007, 32 (12-13), pp.1079-1093. 10.1080/08927020601013817 . hal-00515006

\section{HAL Id: hal-00515006 \\ https://hal.science/hal-00515006}

Submitted on 4 Sep 2010

HAL is a multi-disciplinary open access archive for the deposit and dissemination of scientific research documents, whether they are published or not. The documents may come from teaching and research institutions in France or abroad, or from public or private research centers.
L'archive ouverte pluridisciplinaire HAL, est destinée au dépôt et à la diffusion de documents scientifiques de niveau recherche, publiés ou non, émanant des établissements d'enseignement et de recherche français ou étrangers, des laboratoires publics ou privés. 


\section{Molecular Simulation}

Journal of

Experimental Nanoscience

- Taylor \& Francis

Taylor Sfrancis Group

\section{Application of Molecular Dynamics DL_POLY Code to Interfaces of Inorganic Materials}

\begin{tabular}{|c|c|}
\hline Journal: & Molecular Simulation/Journal of Experimental Nanoscience \\
\hline Manuscript ID: & GMOS-2006-0091.R1 \\
\hline Journal: & Molecular Simulation \\
\hline $\begin{array}{r}\text { Date Submitted by the } \\
\text { Author: }\end{array}$ & 07-Aug-2006 \\
\hline Complete List of Authors: & $\begin{array}{l}\text { Martin, Paul; University of Bath, Chemistry } \\
\text { Parker, Steve; University of Bath, Chemistry } \\
\text { Spagnoli, Dino; University of Bath } \\
\text { Marmier, Arnaud; University of Bath } \\
\text { Sayle, Dean; Academy of the United Kingdom, Cranfield University } \\
\text { Watson, Graeme; Trinity College Dublin }\end{array}$ \\
\hline Keywords: & DL_POLY, Molecular Dynamics, Interfaces \\
\hline
\end{tabular}

\section{SCHOLARONE ${ }^{\text {m }}$} Manuscripts 


\title{
Application of Molecular Dynamics DL_POLY Codes to
}

\author{
Interfaces of Inorganic Materials
}

Paul Martin, Dino Spagnoli, Arnaud Marmier, Stephen C. Parker.

Department of Chemistry, University of Bath, Claverton Down, Bath, UK

BA2 7AY. E-mail: s.c.parker@bath.ac.uk: Fax: +44 1225 386231, Tel: +44

1225386505

Dr. Dean C. Sayle Defence Academy of the United Kingdom, Cranfield

University Shrivenham, Swindon United Kingdom SN6 8LA. E-mail:

d.c.sayle@cranfield.ac.uk Fax:+44 (0)1793 785772 , Tel:+44 (0)1793

785264

Dr. Graeme Watson School of Chemistry, University of Dublin, Trinity

College, Dublin 2. E-mail: watsong@ @tcd.ie Fax:+353 (0)1 6712826 Tel:

$+353(0) 16081357$

\begin{abstract}
Three recent applications of the DL_POLY molecular dynamics code are described, which demonstrate the flexibility and viability of the code for extending our understanding of the structure, stability and reactivity of ceramics and minerals at the atomic level. The first is an investigation into differences in oxygen atom mobility in bulk and at the most stable $\{111\}$ surface of ceria. The results show enhanced surface transport but that it is via subsurface oxygen. Secondly, we investigate how polychlorodibenzo-pdioxins (PCDDs) molecules might adsorb on clay surfaces. The resulting adsorption energies show a clear relationship with chlorine content of the molecule. Finally, we apply DL_POLY to comparing the aggregation of magnesium oxide and calcium carbonate nanoparticles. We find that very small calcium
\end{abstract}


carbonate nanoparticles are amorphous and their aggregation shows no preferred orientation in contrast to magnesium, which remain highly crystalline and combine in a highly structural specific way.

\section{Introduction}

The aim of this paper is to describe our current work using DL_POLY to model the structures and energetics of inorganic solids, with particular emphasis on surfaces and nanostructures. We illustrate the scope of DL_POLY to modelling surfaces and interfaces by considering three recent examples. The first example describes our application of DL_POLY to explore the stability and transport of surface defects, oxygen vacancies on the most stable surface of ceria. Understanding the factors controlling the formation of surface defects is of importance to understanding surface properties. As it is the surface defect that is often the site of chemical activity. Another component of chemical reactivity is understanding how molecules might adsorb onto surfaces. Here we are following the DFT work by the Cambridge group [1] by applying DL_POLY to help our understanding of these processes. Nanoparticles are of great scientific interest as they are effectively a bridge between bulk materials and atomic or molecular structures. The interesting and sometimes unexpected properties of nanoparticles are partly due to their large number of surface sites aspects. The percentage of atoms at the surface of a material becomes significant as the size of that material approaches the nanoscale. Thus in our final section we describe we describe recent work on studying the properties of nanoparticles.

\section{Molecular Dynamics at the Stable Low Index $\{111\}$ Surface of Ceria}


Materials based on ceria $\left(\mathrm{CeO}_{2}\right)$ are used in the production and purification of hydrogen, the purification of exhaust gases in three-way automotive catalytic converters, and other catalytic application [2-5]. These applications make use of the electrochemical properties of this ceria: the ability to shift between its two oxidation states, $\mathrm{Ce}(\mathrm{III})$ and $\mathrm{Ce}(\mathrm{IV})$, and the high mobility of bulk oxygen species - properties that allow ceria to behave as an oxygen buffer. It is important therefore that we understand more fully how oxygen atoms move in the ceria surface, just under the surface and in bulk. In this study, we focused on studying the $\{111\}$ surface of ceria, which is calculated to be the stable [8] and hence most prevalent.

The METADISE code (minimum energy techniques applied to dislocation, interface, and surface energies) [6] was used to construct surface slabs for DL_POLY.

A slab of the $\{111\}$ surface was constructed to a thickness of $20.6 \AA$, that is sufficiently thick to allow realistic simulation of bulk ceria at the centre of the slab. The slab contains 996 atoms. $332 \mathrm{Ce}, 664 \mathrm{O}$. We chose to use 3D periodicity for computational ease and hence introduced a $50 \AA$ vacuum gap. The Coulombic sums were calculated using Ewald summation precision of $10^{-5}$. The potential model used in this work is a potential from Balducci et al (1997) [7] that is a modification of a potential derived by Sayle T.X.T. (1994) [8]. We included the shell model of Dick and Overhauser [11] to simulate ionic polarizability of the oxygen ions. In this model, the oxygen ion is represented as a core plus a shell coupled by a harmonic spring. The total charge is separated between the shell and the core. In the molecular dynamics simulations the shells were given a mass of 0.5 au. 
In order to investigate oxygen atom mobility at the stable $\{111\}$ ceria surface a potential of mean force (PMF) method modification to DL_POLY has been used to simulate the pathway and variation in free energy of a single oxygen atom migrating in bulk ceria and also at surface.

The constrained atom is moved towards a vacancy at a constant velocity. The velocity is usually $0.0001 \mathrm{~nm}$ per fs.

$$
\Delta A(z)=A(z)-A\left(z_{0}\right)=\int_{z_{0}}^{z} f_{z} d z
$$

$f_{\mathrm{z}}$ : average force in direction of vacancy.

Once energetically minimised, vacancies were introduced in to the slab. The modification now allows a single atom to be constrained so that it is forced to migrate to the vacancy position, at a pre-set velocity. The constrained atom is given a target vector, which is the co-ordinates of the lattice vacancy position. However, the constrained atom is free to move perpendicular to the target vector. The average force of the constrained atom along the target vector is recorded at the end of each time-step. Integration of the force with respect to distance yields the free energy, shown in equation (1), $\Delta A(z)$ is the free energy change of migration, $A(z)$ is the free energy at position $z$ and $A\left(z_{0}\right)$ is the free energy at the initial position.

Thus the free energy of migration of the atom to be calculated at various temperatures. Additionally, MD simulation can be run just above zero Kelvin to enable the identification of the pathway and energy change neglecting temperature. This allows for simple (or an initial) analysis of the migration process, without involving complications 
of nearby atoms excessively hindering the constrained atom during its trajectory. In doing this, entropy effects are ignored, and therefore the energy of migration rather than the free energy of migration, is in fact calculated.

Migration of a single oxygen atom was investigated at the centre of the slab, which represents bulk ceria; just beneath the surface, with an oxygen migrating between bulk and a vacancy position just under the surface; and at the surface, where oxygen migration is simulated between the layer of oxygen atoms just beneath the surface and the surface layer, and also the migration from a surface position to a near surface vacancy.

\section{Oxygen Migration}

The first PMF simulations were carried out on the diffusion of oxygen in the bulk of the slab. The slab was initially run at $300 \mathrm{~K}$ using the nvt ensemble, and the migration path plotted. Figure 1 shows that the pathway between oxygen sites was linear and the energy profile in figure 2 shows that the maximum is the energy of migration was $0.66 \mathrm{eV}$ and that the position was half way between the oxygen sites. The activation energy agrees very well with the work of Balducci et al.(1997) [9] who calculated $0.65 \mathrm{eV}$ using a series of static energy calculations.

We can check that the location of the transition state is exactly halfway between the sites by plotting the average force of the constrained atom in the direction of travel in Figure 3. The force passes through zero at $1.05 \AA$ along a total distance of $2.07 \AA$.

We next modelled the trajectory for an oxygen atom migrating from the oxygen layer just below the surface to a vacant site on the surface.

Figure 4 shows the pathway. The first point to notice is the trajectory is now curved. 
The energy plot, figures 5 shows also a marked asymmetry. The transition state in this case is very close to the surface $\sim 0.5 \AA$. Furthermore, Figure 5 shows that the activation energy for the oxygen migration from the surface to the level just beneath is considerably smaller ie. $0.06 \mathrm{eV}$. Interestingly, a greater activation energy, $0.3 \mathrm{eV}$, is required to activate the return of a subsurface oxygen directly to the $\{111\}$ surface from this layer beneath, which is still less than $0.66 \mathrm{eV}$, the bulk value. It also clearly demonstrates that the vacancy is more stable just below the surface.

The next stage was to examine the transport from the surface layers to the bulk. Figure 6 suggests the barrier is $0.67 \mathrm{eV}$, with the sub-surface site $0.2 \mathrm{eV}$ less stable.

Analysis of the trajectory shows that the migration path is not linear as in the bulk simulation, and that the position of the transition state is predicted to be slightly closer to the bulk layer than the sub-surface layer.

We also considered the migration of an oxygen atom across the surface and surprisingly found that the activation energy was $2.5 \mathrm{eV}$. Thus Figure $7 \mathrm{a}$ and $7 \mathrm{~b}$ illustrates how a migrating oxygen atom would move down a layer just under the surface, and then would return to the surface layer leaving a vacancy in the sub-surface layer, which is the most stable site for an isolated vacancy, rather than transport across the surface.

We have seen more complex behaviour in some of the simulations, but still involving oxygen movement between the top two layers. Figure 8 shows the initial configuration of a concerted mechanism involving an oxygen atom from the surface layer constrained to move down into a vacancy position just under the surface. The initial constraint is shown by the largest green arrow in order. The constrained migration results in the formation of 
a vacancy in a less stable position on the surface because vacancies are more stable at a position just under the surface. The result is that, as the constrained oxygen leaves its lattice site to form a vacancy the next oxygen atom fills the site and because the energy barriers are low we find that the next few oxygens move in unison. The next result is that a sub-surface vacancy is formed but can be potentially some distance from the original vacancy.

The concerted motion does however highlight one problem, namely that the movement of the ions in the concerted mechanism causes a resistance to the movement of the constrained atom, thus the resulting free-energy change is the sum of the activation free energies of migration and the free energy for overcoming the drag-force. Thus care must be taken in interpreting the activation free energy.

Another important issue is the effect of charge compensation particularly when the charge compensating defects are bound to the oxygen vacancies. We consider them in the following section.

We treated the charge compensating defects as localised electrons on neighbouring cerium ions. Thus the first instance considered (Figure 9a) is when the oxygen atom migrates directly into a vacant site which is adjacent to two $\mathrm{Ce}^{3+}$ ions. The trajectory of migration takes the oxygen between the two adjacent $\mathrm{Ce}^{3+}$ ions. The effect of the charge compensating $\mathrm{Ce}^{3+}$ ions on the migration is significant. The new activation energy is 1.35 $\mathrm{eV}$ (Figure 10) for the oxygen migration up and between the compensating ions, compared to $0.06 \mathrm{eV}$ for similar migration without the involvement of compensating ions. 
Another interesting result of this calculation is that the final configuration, with the vacancy just below the surface is calculated to be $0.4 \mathrm{eV}$ less stable. But note that the final oxygen vacancy position is not in the highly bound state (i.e.) just under the surface and adjacent to the charge compensating $\mathrm{Ce}^{3+}$ ions.

The second example (Figure 11a) considered is where an oxygen atom just beneath the surface is forced to migrate to the surface, adjacent to and to the side of both the vacancy and a compensating $\mathrm{Ce}^{3+}$ ion, (ie.) the oxygen vacancy moves from the top layer to the layer underneath.

In this instance, the activation energy of migration (Figure 12) is similar to that for the un-charged compensated migration, (ie) $0.10 \mathrm{eV}$ cf. $0.06 \mathrm{eV}$.

The activation energy for the reverse process is approximately $1.0 \mathrm{eV}$, thus in common with the un-charged compensated migration, the vacancy is more stable below the surface but is very much more stabilised i.e., by about $0.9 \mathrm{eV}$.

Hence the energetics for the migration of an oxygen atom into a bound charge compensated vacancy cluster, when that oxygen atom approaches the vacancy from the side of the compensation, differ significantly from the case of migration between the compensating ions.

The final example considered (Figure 13a) involves the migration of an oxygen atom, which is originally under the surface between two adjacent $\mathrm{Ce}^{3+}$ ions, to a vacancy position set just to the side of this charge compensating pair of ions. The direction of migration is away from the charge compensation up into the vacancy which is considered 
here only to be partially bound because the vacancy is not symmetrically configured. Thus the final configuration leaves the vacancy in the highly bound state.

The plot in figure 14 shows that the activation energies are still small, at $0.25 \mathrm{eV}$ for the forward and $0.4 \mathrm{eV}$ for the reverse.

However, activation migration energy is 4 times greater than the energy for the uncharged compensated case. It is 2.5 times greater than the migration into a bound charge compensated vacancy with a trajectory from the side, but is much smaller than the $1.35 \mathrm{eV}$ required for activation of migration up and between charge compensating ions into the fully bound vacancy.

The results show that energetically the vacancy prefers to be bound to the charge compensating defects in the subsurface oxygen site. Furthermore, the location of these charge compensating defects modify the activation energies and hence the mobility of oxygen near the surface. In the case of the movement along the site of the $\mathrm{Ce}^{3+}$ ions, the effect is significant and will have a large anisotropic effect on the atom transport.

The results therefore suggest that the migration of a defect cluster differs from isolated defects. Therefore, it is important to investigate the stability of different clusters.

These simulation results suggest an important significance in the direction of migration around or between partially or fully bound charge compensated defects, in that it appears that oxygen atoms prefer to migrate up from the side of a bound compensated surface vacancy. 


\title{
Organic molecules adsorbed on clay surface: DL_POLY as a high-throughput engine
}

\author{
Organic pollutants and related molecules can pose a serious environmental threat to the \\ global ecosystem. Amongst the most notorious are these halogenated substances used as \\ pesticides or by-products from human activities. Concerns are raised due to their general \\ toxicity and the fact they can enter human and animal food chains, as well as affecting \\ the quality of water or soil or atmosphere. In the absence of well thought remediation \\ strategies, risk management information is needed to allow stakeholders to decide options \\ concerning disposal.
}

Our interest lies in understanding how these molecules bind to mineral surfaces and to establish a high-throughput methodology to efficiently investigate many molecules/substrates combinations.

As a starting point, we choose the polychloro-dibenzo-pdioxins (PCDDs) family. The 76 members of this familly (congeners) have a different number of chlorine atoms replacing hydrogens at different positions.

We choose a substrate with soil relevance, a clay. The di-octahedral 2:1 sheet silicate pyrophillite, $\mathrm{Al}_{4} \mathrm{Si}_{8} \mathrm{O}_{20}(\mathrm{OH})_{4}$. is a simple example of the family of clay minerals that form a significant fraction of many soil and rock types. Its [001] face was first considered. 
Preliminary DFT calculations showed that the organic molecules do not chemisorb on the pyrophillite surface. Of course, DFT is not adapted to the accurate study of physisorption phenomena due to its poor treatment of dispersion. Therefore our potential based method was deemed ideal for such a study.

We now describe the choices of models, tools and resources, followed by a brief discussion of the automatisation procedures and comments on the scientific results.

\section{Model}

A classical model requires an electrostatic description of atoms as well as a parameterisation of the intra-molecular and the inter-molecular interactions.

Well established models for Zeolite and clay minerals make modeling the substrate a comparatively straightforward task. We chose the parameterisation of Lewis and Catlow [10] in which the electrostatics is described by the shell model of Dick and Overhauser [11]. The total charge is separated between the shell and the core. In the molecular dynamics simulations the shells were given a mass of $0.5 \mathrm{au}$.

For the molecules, we chose the CVFF model [12] for the bonding interactions. The attribution of the electrostatic components was more complex. We used ab initio calculations (gaussian code [13]) with the Dunning/Huzinaga valence double-zeta basis set on selected PCDDs'. From these we extracted the charge distribution to extrapolate point charges for all the PCDD. This simplified charge model is described in table 1. 


\begin{tabular}{lc}
\hline \hline \multicolumn{1}{c}{ Specie } & Charge $(\mathrm{e})$ \\
\hline $\mathrm{H}$ & .20 \\
$\mathrm{Cl}$ & -.03 \\
$\mathrm{O}$ & -.50 \\
$\mathrm{C}(\mathrm{H}$ bonded $)$ & -.20 \\
$\mathrm{C}(\mathrm{Cl}$ bonded $)$ & .03 \\
$\mathrm{C}(\mathrm{O}$ bonded $)$ & .25 \\
\hline \hline
\end{tabular}

Table 1: Charge model (in e)

We generated the interaction parameters between the substrate and the adsorbed molecules by using the standard combination rules, which is a valid approach to obtain dispersion-repulsion interaction coefficients in the absence of strong bonding.

Our confidence in this model is confirmed by the excellent agreement of test calculations on the adsorption of simpler molecules (linear and cyclic aliphatic hydrocarbons) with experimental measurements $[14,15]$ : in the case of cyclohexane, $-57 \mathrm{~kJ}^{\mathrm{mol}}{ }^{-1}$ compared to $-54 \mathrm{~kJ} \cdot \mathrm{mol}^{-1}$ at $423 \mathrm{~K}$.

\section{DL_POLY as an optimiser}

DL_POLY is the UK's national flagship package for molecular dynamics (MD) simulations [16]. While it is especially good for simulating very large systems, its modular structure makes it very adaptable. It supports some form of energy optimisation (albeit not as efficiently as specialist codes such as GULP [17] or METADISE [6]). It is also $\mathrm{cml}$ enabled which allows for certain direct inter-operability with some DFT codes.

The choice of a MD code for a problem essentially based on optimisation might appear odd at first. This choice was justified because the force field we designed is relatively complex and not adaptable to GULP or METADISE codes without heavy recoding and especially because that our main objective is to tackle adsorption in realistic conditions, i.e. at various temperatures and with of water. Molecular Dynamics is the tool of choice for these more advanced problems. So, to maintain upward compatibility, we decided to 
use the same code for the current study as we would for the more advanced one, at the cost of the early added complexity of using a MD code to perform optimisation

\section{UCL Condor Pool}

This project is combinatorial in nature. Not only are there seventy-six non equivalent PCDD molecules, but the determination of reliable adsorption sites requires multiple starting configurations. Furthermore, the search space is large and the potential energy surface can be complex and contain many local minima. However, the systems are not overly large and each energy calculation is not too demanding, therefore, a heavy handed, brute force approach to optimisation is affordable.

Instead of using a supercomputer, we took advantage of the UCL Condor pool. This type of resource is ideal for this study, as it contains a very large amount of processors (at the time of writing 960 CPUs, operating under Windows OS).

Another feature of the Condor setup is the DAGMAN workflow manager which allows complex workflows. In our case, it essentially made the analysis task automatic.

\section{Methodology}

First of all, three sets of independent calculations are required: molecule only (M), substrate only $(\mathrm{S})$, molecule adsorbed on substrate $(\mathrm{M}+\mathrm{S})$. The adsorption energy is defined as a difference between the energy of these systems $\left(\mathrm{E}_{\mathrm{MS}} \mathrm{EM}^{-}-\mathrm{E}_{\mathrm{S}}\right)$

The two custom files node_list and atom_list are at the centre of the data structure and operate on the two modified DL_POLY input files FIELD and CONFIG. node_list contains seventy six lines, one for each PCCD, starting with a user provided molecule code, and then twenty two intermediate atom codes, in the order in 
which the atoms appear in the CONFIG file (these codes can be $\mathrm{H}, \mathrm{Cl}, \mathrm{O}, \mathrm{C}, \mathrm{CH}, \mathrm{CCl}$ or CO). atom_list creates a link between these intermediary codes and the names used in the CONFIG $(\mathrm{H}, \mathrm{Cl}, \mathrm{O}, \mathrm{C})$, as well as with the charges appearing in the FIELD file (as the carbon atoms can be allocated different charges). It also contains information concerning the bonding parameters, which need to be allocated in the FIELD file.

A list of dedicated scripts reads the node_list and operates on the 76 corresponding directories.

The simpler scripts are just expansion of unix commands (list_cp, list_mv, list_grep). list_setdir generates the data structure (directories NODE_nmoleculecode, where $\mathrm{n}$ is an arbitrary molecule index, corresponding to the order in node_list). list_config replaces <list> flags in a modified CONFIG by the correct atom name and put the correct CONFIG in its directory. list_field replaces <list> and < harm> flags in the field by their value from the atom_list (atom name and charge, two specie bonds parameters). list_fire submit the jobs to the condor scheduler (via run.dlp files). list_rota, is an expanded version of list_config which additionally applies a rotation and/or translation to the flagged atoms of CONFIG (in our case of course those of the PCDD molecule).

These scripts are sufficient to optimise the structures of the isolated molecules.

As already mentioned, the case of the adsorbed molecules is slightly more complex as several starting configuration are needed. Each of these is carried sequentially, for each molecule. The script config_rota, a same directory, command line version of 
list_rota is used in conjunction with DAGMAN. list_fire is also updated to list_fire_dag. Practically, three sites of high symmetry were explored (above Si, above O, above "hexagon" centre, see figure 16) with 8 rotational configuration (by increment of pi/2), with 24 calculations per molecule.

\section{Results and discussion}

In Figure 15 we plot the adsorption energy of the 76 PCDDs. The molecule index is only meaningful in so far as it increases with the number of chlorine atoms. The horizontal lines are a guide for the eye and correspond to molecules with the same number of chlorine. It can be seen that the adsorption energy is strongly correlated to the number of chlorine. Each additional chlorine attached to the carbon rings leads on average to an increase of $.03 \mathrm{eV}$ in adsorption energy.

Furthermore, and as could have been expected, all the molecule lay flat on the surface, see Figure 16.

We also observe that the corrugation do increase with the number of chlorine. The potential energy surface is very flat for the PCDD with a low $\mathrm{Cl}$ count (indeed a translational corrugation of $0 \pm .01 \mathrm{eV}$ for $\mathrm{C}_{12} \mathrm{O}_{2} \mathrm{H}_{8}$ ). At higher $\mathrm{Cl}$ count, the corrugation increases, up to $.07 \mathrm{eV}$ for $\mathrm{C}_{12} \mathrm{O}_{2} \mathrm{Cl}_{8}$. Therefore, the concept of adsorption site is rather meaningless for $\mathrm{NCl}$ lower than 4. For $\mathrm{NCl}$ higher than 3, the PCDD almost systematically favours a position such as illustrated in Figure 16, with the centre of mass of the molecule situated above a surface oxygen, and where the benzene rings sit inside the quasi-hexagon of the surface top layer. 


\begin{abstract}
In addition, to modelling molecules in contact with surfaces we are also concerned with the interaction of nanoparticles.
\end{abstract}

\title{
Molecular dynamics simulations of the structural changes that occur during aggregation of mineral oxide and carbonate nanoparticles
}

DL_POLY has been used by other groups to study the structure and stability on minerals on the nano-scale. Spano et al found computational evidence of $\mathrm{ZnS}$ forming bubble type clusters [18] $(\mathrm{ZnS})_{\mathrm{n}}$ clusters, with $\mathrm{n}$ ranging from 10 to 47 , were energy minimised using a simulated annealing technique. Spano et al found that spheroidal (bubble) clusters with only three coordinated atoms are more stable than the bulk structure. If the number of $\mathrm{ZnS}$ units is increased to 60 then the appearance of onion structures occur [19]. This is a $(\mathrm{ZnS})_{12}$ inside a bubble of $(\mathrm{ZnS})_{48}$. Banfield and co workers have also considered $\mathrm{ZnS}$ nanoparticles, typically $\mathrm{n}=360-400$, making the diameter of the $\mathrm{ZnS}$ crystal around $3 \mathrm{~nm}$, which is comparable to experimental synthesised particles [20]. The Banfield group started using molecular dynamics to study the phase stability of $\mathrm{ZnS}$ nanoparticles in vacuum and in the presence of water. They found that the wurtzite $(\mathrm{ZnS})$ nanoparticles smaller than $7 \mathrm{~nm}$ are more stable than sphalerite nanoparticles in vacuum at $300 \mathrm{~K}$. However adsorption of water onto the $\mathrm{ZnS}$ nanoparticle surface stabilizes the sphalerite structure. Zhang et al [21] predicted anhydrous $\mathrm{ZnS}$ nanoparticles to have a highly disordered structure. Again these nanoparticles had a diameter of around $3 \mathrm{~nm}$. The adsorption of water, however, onto the nanoparticle surface results in the formation of a 
relatively crystalline core. Zhang and Banfield showed that this phase transformation of sphalerite to wurtzite could be caused by nanoparticle aggregation and coarsening [22]. These $3 \mathrm{~nm} \mathrm{ZnS}$ nanoparticles were randomly orientated with respect to each other and allowed to aggregate to form a larger nanoparticle. Following aggregation the coarsened particle adopts a near-amorphous structure that transforms rapidly to wurtzite. This work by Banfield and co-workers has provided a link between atomistic simulation and the forces that cause their aggregation, i.e. colloidal chemistry. We have used DL_POLY to extend the range of materials to simulate the aggregation process of two different materials, $\mathrm{MgO}$ and calcite $\left(\mathrm{CaCO}_{3}\right)$ nanoparticles. First we will describe the potentials used to describe the interatomic interactions and then describe how we set up our systems for aggregation.

The potential model used for simulating the magnesium-oxygen interaction is that of Lewis and Catlow [23] and has been used before to simulate $\mathrm{MgO}$ surfaces [24, 25] and $\mathrm{MgO}$ nanocrystals [26]. The potential model used for simulating the calcium-carbonate interactions in calcite is that of Pavese et al [27] and has been used in the past to simulate calcite surfaces [28] and also calcite nanoparticles [29]. All simulations in this section employ the shell model of Dick and Overhauser [11]. Where the shells are in the oxygen species only and have a mass of 0.2 au in $\mathrm{CaCO}_{3}$ and 0.5 in $\mathrm{MgO}$, this represents the electronic charge cloud connected by a harmonic spring to a core containing most of the anion's mass.

We have begun studying the structure and aggregation using molecular dynamics simulations by modelling an eight nanoparticle system. Each of the nanoparticles in the simulation is the same distance apart. The simulation was performed in vacuum with no 
periodic boundary conditions. The molecular dynamic simulation was performed in a canonical ensemble at $300 \mathrm{~K}$ and ambient pressure using a time step of $0.1 \mathrm{fs}$. Each simulation was run for $1 \mathrm{~ns}$ with a 2 ps equilibration period.

\section{Aggregation of Magnesium Oxide nanoparticles}

Each $\mathrm{MgO}$ nanoparticle had a $0.6 \mathrm{~nm}$ in diameter and the simulation started with them being $2.3 \mathrm{~nm}$ apart. The starting configuration is shown in figure 17. We have 8 cubes of $\mathrm{MgO}$ as the most stable surface grows along the $\{100\}$ plane [6]. We can plot the potential energy of our simulation as it evolves over time (figure 18). The starting configuration has a potential energy of $-482.1 \mathrm{~kJ} / \mathrm{mol}$ and then drops rapidly in-between $30-55 \mathrm{ps}$ to $-484.4 \mathrm{~kJ} / \mathrm{mol}$. This decrease in potential energy corresponds to the initial aggregation of $\mathrm{MgO}$ nanoparticles. Figure 19 shows a snapshot of the MD simulation at 30ps. The particles retain their bulk-like structure and well ordered surfaces. At 30ps single $\mathrm{MgO}$ nanoparticles are starting to aggregate together in pairs. The aggregation process seems very ordered and is dominated by orientation along the $\{100\}$ plane. This snapshot is also interesting because it also shows that the merging nanoparticles will join at the corners first. The pair of nanoparticles that are circled in figure 19 are a pair that drifts further and further away from the rest of the nanoparticles and don't aggregate with the other six nanoparticles. Since they are so far away in some of the snapshots it is not practical to show them in some of the pictures. At 60ps figure 20 shows a stabilisation of potential energy at around $-484.4 \mathrm{~kJ} / \mathrm{mol}$. Figure 20 shows a snapshot of the MD simulation at $60 \mathrm{ps}$ and shows the aggregation of a single $\mathrm{MgO}$ nanoparticle to a pair. The snapshot again illustrates the general result that aggregation has initially occurred along 
the corners of the nanoparticles. The nanoparticles find their preferred face centre cubic site at 220ps (figure 21) when the potential decreases to around $-484.6 \mathrm{~kJ} / \mathrm{mol}$. The increased ordering of aggregation along the $\{100\}$ plan continues when the potential energy decreases to $-485.8 \mathrm{~kJ} / \mathrm{mol}$ when a pair of nanoparticles aggregate to the triplet at 275 ps (figure 22). The final configuration occurs at 460ps (figure 23). Here the sixth nanoparticle joins the cluster of five. The two nanoparticles circled in Figure 19 were in fact too far away to become attracted to the rest of them. This aggregation process has also shown that when crystal growth occurs in $\mathrm{MgO}$ there are the chances of kinks and steps being formed.

We can compare any structural changes that may have occurred during aggregation by comparing the radial distribution functions (RDF) of aggregated nanoparticles with bulk $\mathrm{MgO}$. Figure 24 shows the RDF of the (a) aggregated $\mathrm{MgO}$ nanoparticle and (b) bulk $\mathrm{MgO}$. The RDF's of the aggregated $\mathrm{MgO}$ nanoparticle and bulk $\mathrm{MgO}$ are very similar with the first three peaks showing $\mathrm{Mg}-\mathrm{O}$ bond distances at $0.21,0.35$ and $0.45 \mathrm{~nm}$ in both graphs. These interatomic distances agree with x-ray diffraction experiments $[30,31]$. The RDF of the aggregated nanoparticle shows that during aggregation the $\mathrm{MgO}$ nanoparticles continue to maintain their crystalline form. Furthermore, the bonding between the $\mathrm{MgO}$ is so highly directed, resulting in a continuous structure. Thus although the overall shape is non-symmetric there are no obvious grain boundaries being formed. Next we show the aggregation process of eight calcite nanoparticles.

\section{Aggregation of calcite nanoparticles}


Eight calcite nanoparticles that are $1.6 \mathrm{~nm}$ in diameter are placed $1.7 \mathrm{~nm}$ apart. The starting configuration is shown in figure 25 . The simulation was run for $1 \mathrm{~ns}$. Figure 28 shows the potential energy of the system as a function of MD time. The simulation starts with a potential energy of $-715.3 \mathrm{~kJ} / \mathrm{mol}$. As the simulation evolves the first drop in potential energy occurs at around 50ps. This corresponds to figure 27 which shows that before aggregation occurs the individual nanoparticles will relax on their own to a potential energy of $-715.6 \mathrm{~kJ} / \mathrm{mol}$, which corresponds to a more disordered structure. Once the calcite particles are relaxed aggregation occurs in pairs. At around 67ps the eight nanoparticles have formed four nanoparticles and the energy lowers to $716.9 \mathrm{~kJ} / \mathrm{mol}$. Figure 28 shows a snapshot of aggregation at $87 \mathrm{ps}$. The potential energy of this system continues to lower, $-718.2 \mathrm{~kJ} / \mathrm{mol}$, as the four calcite nanoparticles start to form two larger nanocrystals. The two larger calcite nanoparticles now become attracted to each other and again as they merge the potential energy drops to $-719.9 \mathrm{~kJ} / \mathrm{mol}$ at around 140ps (figure 29. The final configuration occurs at 180ps and the potential energy stabilises at $-721.0 \mathrm{~kJ} / \mathrm{mol}$. Figure 30 shows the aggregated nanoparticle, which has lost its symmetry. The final nanoparticle has a longest diameter of $4.17 \mathrm{~nm}$ and a width of $1.816 \mathrm{~nm}$. Figure $31 \mathrm{a}$ shows the RDF of the final aggregated calcite nanoparticle. When compared with the RDF of bulk calcite (Figure 31b) it has much less order and is more amorphous then the crystalline bulk. The peaks in the RDF bulk calcite shows the same Ca-O bond distances, $0.23,0.33,0.41 \mathrm{~nm}$, as found experimentally [32, 33]. The average coordination number for a calcium ion in the aggregated nanoparticle is calculated to be around 6.5, which is slightly higher then the coordination number for bulk calcite which 
is 6. This suggests that the amorphous nanoparticle is more closely related to calcite, rather than aragonite which has a calcium ion coordination number of 8 .

The difference between an aggregated calcite and an aggregated $\mathrm{MgO}$ is that $\mathrm{MgO}$ keeps its bulk-like structure and still has crystalline characteristics. The RDF shows sharp peaks whereas the RDF of the aggregated calcite is more amorphous with only two peaks. The atoms of the calcite nanoparticle seems to be more disordered and has no structural preference for linking different particles whereas the aggregated $\mathrm{MgO}$ has a very uniform structure.

There has been a great deal of debate in the literature suggesting that biomineralisation occurs from amorphous calcium carbonate [34-37]. We have shown that very small calcium carbonate nanoparticles are more stable when amorphous and also when they aggregate together their morphology changes drastically. Therefore this aggregation process could promote the exotic morphologies seen in biomineralistion. In future studies we set out to quantify this aggregation process.

\section{Conclusions}


DL_POLY has great scope for our molecular dynamics simulation experiments. We have been able to calculate both the free energy of nanoparticle aggregation and the activation free energy of individual atom migration. DL_POLY has also been shown to be versatile when used as an energy optimiser when investigating adsorption of molecules on surfaces.

Application of DL_POLY has allowed us to investigate oxygen atom migration at the stable $\{111\}$ surface of ceria. The oxygen migration is shown to be much more energetically favoured at the $\{111\}$ surface than the bulk when via subsurface oxygen vacancies, and the activation free energy of migration was found to be the same in all directions. When considering the effect of neighbouring charge compensating $\mathrm{Ce}^{3+}$ ions the oxygen was found to be less mobile and no longer isotopic.

We have determined the adsorption energy and sites of the 76 PCDD congeners on a pyrophilite surface, using DL_POLY. The molecules are weakly bonded and the corrugation is low. The variation in energy with adsorption sites is not small. The main factor controlling the adsorption energy is the number of chlorine atoms.

The real simplicity of the PCDDs adsorption on this surface means that the process can be modelled very simply and parameterised for use in large scale soil remediation models.

We have investigated the differences between calcite and $\mathrm{MgO}$ nanoparticle aggregation. The difference between an aggregated calcite and an aggregated $\mathrm{MgO}$ is that $\mathrm{MgO}$ keeps its bulk-like structure and still has crystalline characteristics. The RDF shows sharp peaks whereas the RDF of the aggregated calcite is more amorphous with only two peaks. The 
2

structure of the calcite nanoparticle seems to be more mobile whereas the aggregated $\mathrm{MgO}$ has a very uniform structure.

In summary, DL_POLY is allowing us to carry-out sophisticated molecular dynamics simulations, intricate computational calculations and gives us scope for further investigatory work. Importantly, these applications of DL_POLY are providing a useful complement to experiments. 


\section{References:}

1. White, T.O., R.P. Bruin, J. Wakelin, C. Chapman, D. Osborn, P. Murray-Rust, E. Artacho, M.T. Dove, and M. Calleja. eScience methods for the combinatorial chemistry problem of adsorption of pollutant organic molecules on mineral surfaces. Proceedings of the UK e-Science All Hands Meeting 2005. (2005)

2. Deluga, G.A., J.R. Salge, L.D. Schmidt, and X.E. Verykios. Renewable hydrogen from ethanol by autothermal reforming. Science. 303. 5660. (2004)

3. Otsuka, K., T. Ushiyama, and I. Yamanaka. Partial Oxidation of Methane Using the Redox of Cerium Oxide. Chemistry Letters. 9. (1993)

4. Trovarelli, Catalysis by Ceria and Related Materials. 2002, London: Imperial College Press.

5. Park, S.D., J.M. Vohs, and R.J. Gorte. Direct oxidation of hydrocarbons in a solid-oxide fuel cell. Nature. 404. 6775. (2000)

6. Watson, G.W., E.T. Kelsey, N.H. deLeeuw, D.J. Harris, and S.C. Parker. Atomistic simulation of dislocations, surfaces and interfaces in $\mathrm{MgO}$. Journal of the Chemical Society-Faraday Transactions. 92. 3. (1996)

7. Balducci, G., J. Kaspar, P. Fornasiero, M. Graziani, M.S. Islam, and J.D. Gale. Computer simulation studies of bulk reduction and oxygen migration in $\mathrm{CeO} 2-$ ZrO2 solid solutions. Journal of Physical Chemistry B. 101. 10. (1997)

8. Sayle, T.X.T., S.C. Parker, and C.R.A. Catlow. Surface Segregation of Metal-Ions in Cerium Dioxide. Journal of Physical Chemistry. 98. 51. (1994)

9. Balducci, G., M.S. Islam, J. Kaspar, P. Fornasiero, and M. Graziani. Bulk reduction and oxygen migration in the ceria-based oxides. Chemistry of Materials. 12. 3. (2000)

10. Lewis, G.V. and C.R.A. Catlow. Potential Models for Ionic Oxides. Journal of Physics C-Solid State Physics. 18. 6. (1985)

11. Dick, A.W. and B.G. Overhauser. Theory of dielectric constants of alkali halide crystals. Physical Reviews. 112. 1. (1958)

12. Dauberosguthorpe, P., V.A. Roberts, D.J. Osguthorpe, J. Wolff, M. Genest, and A.T. Hagler. Structure and Energetics of Ligand-Binding to Proteins Escherichia-Coli Dihydrofolate Reductase Trimethoprim, a Drug-Receptor System. Proteins-Structure Function and Genetics. 4. 1. (1988)

13. Frisch, M.J., G.W. Trucks, H.B. Schlegel, G.E. Scuseria, M.A. Robb, J.R. Cheeseman, J. Montgomery, J. A., T. Vreven, K.N. Kudin, J.C. Burant, J.M. Millam, S.S. Iyengar, J. Tomasi, V. Barone, B. Mennucci, M. Cossi, G. Scalmani, N. Rega, G.A. Petersson, H. Nakatsuji, M. Hada, M. Ehara, K. Toyota, R. Fukuda, J. Hasegawa, M. Ishida, T. Nakajima, Y. Honda, O. Kitao, H. Nakai, M. Klene, X. Li, J.E. Knox, H.P. Hratchian, J.B. Cross, V. Bakken, C. Adamo, J. Jaramillo, R. Gomperts, R.E. Stratmann, O. Yazyev, A.J. Austin, R. Cammi, C. Pomelli, J.W. Ochterski, P.Y. Ayala, K. Morokuma, G.A. Voth, P. Salvador, J.J. Dannenberg, V.G. Zakrzewski, S. Dapprich, A.D. Daniels, M.C. Strain, O. Farkas, D.K. Malick, A.D. Rabuck, K. Raghavachari, J.B. Foresman, J.V. Ortiz, Q. Cui, A.G. Baboul, S. Clifford, J. Cioslowski, B.B. Stefanov, G. Liu, A. Liashenko, P. Piskorz, I. Komaromi, R.L. Martin, D.J. Fox, T. Keith, M.A. AlLaham, C.Y. Peng, A. Nanayakkara, M. Challacombe, P.M.W. Gill, B. Johnson, 
W. Chen, M.W. Wong, C. Gonzalez, and J.A. Pople, Gaussian 03, Revision C.02. 2004, Gaussian, Inc.: Wallingford CT.

14. Zhukova, A.I., S.V. Bondarenko, and Y.I. Tarasevich. Study of Selectivity of Natural Sorbents with Respect to Hydrocarbons. Ukrainskii Khimicheskii Zhurnal. 42. 7. (1976)

15. Keldsen, G.L., J.B. Nicholas, K.A. Carrado, and R.E. Winans. Molecular Modeling of the Enthalpies of Adsorption of Hydrocarbons on Smectite Clay. Journal of Physical Chemistry. 98. 1. (1994)

16. Smith, W. and T.R. Forester. DL_POLY_2.0: A general-purpose parallel molecular dynamics simulation package. Journal of Molecular Graphics. 14. 3. (1996)

17. Gale, J.D. and A.L. Rohl. The General Utility Lattice Program (GULP). Molecular Simulation. 29. 5. (2003)

18. Spano, E., S. Hamad, and C.R.A. Catlow. Computational evidence of bubble ZnS clusters. Journal of Physical Chemistry B. 107. 38. (2003)

19. Spano, E., S. Hamad, and C.R.A. Catlow. ZnS bubble clusters with onion-like structures. Chemical Communications. 7. (2004)

20. Zhang, H.Z., F. Huang, B. Gilbert, and J.F. Banfield. Molecular dynamics simulations, thermodynamic analysis, and experimental study of phase stability of zinc sulfide nanoparticles. Journal of Physical Chemistry B. 107. 47. (2003)

21. Zhang, H.Z., B. Gilbert, F. Huang, and J.F. Banfield. Water-driven structure transformation in nanoparticles at room temperature. Nature. 424. 6952. (2003)

22. Zhang, H.Z. and J.F. Banfield. Aggregation, coarsening, and phase transformation in $\mathrm{ZnS}$ nanoparticles studied by molecular dynamics simulations. Nano Letters. 4. 4. (2004)

23. Lewis, G.V. and C.R.A. Catlow. Potential model for ionic oxides. Journal of Physical Chemistry: Solid State Physics. 18. (1985)

24. de Leeuw, N.H. and S.C. Parker. Molecular-dynamics simulation of MgO surfaces in liquid water using a shell-model potential for water. Physical Review B. 58. 20. (1998)

25. Harris, D.J., J.H. Harding, and S.C. Parker. Simulations of surfaces and interfaces in MgO. Radiation Effects and Defects in Solids. 151. 1-4. (1999)

26. Sayle, D.C., J.A. Doig, S.A. Maicaneanu, and G.W. Watson. Atomistic structure of oxide nanoparticles supported on an oxide substrate. Physical Review B. 65. 24. (2002)

27. Pavese, A., M. Catti, S.C. Parker, and A. Wall. Modelling of the thermal dependence of structural and elastic properties of calcite, $\mathrm{CaCO}$. Physics and Chemistry of Minerals. 23. 2. (1996)

28. Kerisit, S. and S.C. Parker. Free energy of adsorption of water and calcium on the \{10(1)over-bar-4\} calcite surface. Chemical Communications. 1. (2004)

29. Kerisit, S., D.J. Cooke, D. Spagnoli, and S.C. Parker. Molecular dynamics simulations of the interactions between water and inorganic solids. Journal of Materials Chemistry. 15. 14. (2005)

30. Bragg, W.L. Crystal structure. Nature. 105. (1920) 
31. Tsirel'son, V.G.A., A.S.;Abramov, Yu.A.;Belokoneva, E.L.;Kitaneh, R.;Feil, D. X-ray and electron diffraction study of Mg O. Acta Crystallographica B. 54. (1998)

32. Maslen, E.N.S.t., V.A.;Strel'tsova, N.R. X-ray study of the electron density in calcite, CaCO3. Acta Crystallographica B. 49. (1993)

33. Wyckoff, R.W.G. The crystal structures of some carbonates of the calcite group. American Journal of Science, Serie 50. (1920)

34. Addadi, L., S. Raz, and S. Weiner. Taking advantage of disorder: Amorphous calcium carbonate and its roles in biomineralization. Advanced Materials. 15. 12. (2003)

35. Faatz, M., F. Grohn, and G. Wegner. Amorphous calcium carbonate: Synthesis and potential intermediate in biomineralization. Advanced Materials. 16. 12. (2004)

36. Weiner, S., Y. Levi-Kalisman, S. Raz, and L. Addadi. Biologically formed amorphous calcium carbonate. Connective Tissue Research. 44. (2003)

37. Xu, X.R., J.T. Han, and K. Cho. Formation of amorphous calcium carbonate thin films and their role in biomineralization. Chemistry of Materials. 16. 9. (2004) 


\section{Table of Figures}

Figure 1: Oxygen Atom Migrating in The Middle of $\{111\}$ Surface Slab

Figure 2: Energy Profile of Bulk Oxygen Migration

Figure 3: Plot Showing Constrained Force Experienced by the Migrating Atom over Distance Travelled in Bulk Ceria.

Figure 4: Oxygen Atom Migrating in the $\{111\}$ Surface.

Figure 5: Energy Profile of Oxygen Migration in the $\{111\}$ Surface.

Figure 6: Energy Profile of Oxygen Migration from Bulk to Just Under the $\{111\}$ Surface.

Figure 7 (a) Schematic of oxygen hopping across the surface.

Figure 7 (b) Schematic of oxygen moving through the surface.

Figure 8: Pathway of Concerted Mechanism

Figure 9a: Before migration and with Surface Vacancy in place.

Figure 9b: After Oxygen atom, originally under the surface between $\mathrm{Ce}^{3+}$ ions, has migrated to the surface leaving vacancy just under the surface and between $\mathrm{Ce}^{3+}$ ions.

Figure 10: Energy Profile of Oxygen atom migration between $\mathrm{Ce}^{3+}$ ions onto the Surface.

Figure. 11a: Before migration and with Surface Vacancy in place.

Figure. 11b: After. Oxygen atom, originally under the surface and below the vacancy, has migrated to the surface leaving vacancy just under the surface and below the left $\mathrm{Ce}^{3+}$ ion.

Figure 12: Energy Profile of Oxygen atom migration From under the Left Hand $\mathrm{Ce}^{3+}$ ion into the Surface Vacancy between Compensating $\mathrm{Ce}^{3+}$ ions.

Figure 13a: Before migration and with Surface Vacancy in place.

Figure 13b: After. Oxygen atom, originally under the surface between $\mathrm{Ce}^{3+}$ ions, has migrated to the surface leaving vacancy just under the surface and between $\mathrm{Ce}^{3+}$ ions.

Figure 14: Energy Profile of Oxygen atom migration From under the Left Hand $\mathrm{Ce}^{3+}$ ion into the Surface Vacancy between Compensating $\mathrm{Ce}^{3+}$ ions.

Figure 15: Adsorption energy of the 76 PCDD 
Figure 16: Adsorption position of a PCDD congener. The surface is parallel to the page. The shaded atoms represent the lower layers. Surface: Si Large light grey, O small dark grey. Molecule: $\mathrm{H}$ white, $\mathrm{Cl}$ light grey, $\mathrm{C}$ grey, $\mathrm{O}$ dark grey.

Figure 17: Initial configuration of eight $\mathrm{MgO}$ nanoparticles before aggregation.

Figure 18: Potential energy against time of an MD simulation of $8 \mathrm{MgO}$ nanoparticles

Figure 19: A snapshot of the MD simulation at 30ps for the $\mathrm{MgO}$ system

Figure 20: A snapshot of the MD simulation at $60 \mathrm{ps}$ for the $\mathrm{MgO}$ system

Figure 21: A snapshot of the MD simulation at 220ps for the MgO system

Figure 22: A snapshot of MD simulation at 275ps for the $\mathrm{MgO}$ system

Figure 23: Final configuration of the aggregated $\mathrm{MgO}$ nanoparticles

Figure 24: RDF of the (a) aggregated $\mathrm{MgO}$ nanoparticles and (b) bulk $\mathrm{MgO}$

Figure 25: Initial configuration of calcite nanoparticles for MD simulation

Figure 26: Potential energy against time of an MD simulation of 8 calcite nanoparticles

Figure 27: A snapshot of the MD simulation at 50ps for the calcite $1.6 \mathrm{~nm}$ system

Figure 28: A snapshot of the MD simulation at $87 \mathrm{ps}$ for the calcite $1.6 \mathrm{~nm}$ system

Figure 29: A snapshot of the MD simulation at 140ps for the calcite 1.6nm system

Figure 30: Final configuration of the aggregated calcite nanoparticles

Figure 31: RDF of the (a) aggregated calcite nanoparticles and (b) bulk calcite 


\section{Page 29 of 158}

1

2

3
4

4

5

6
7

8

9

10

11

12
13

14

15

16

17

18

19

20

21

22

23

24

25

26

27

28

29

30

34

35

36

37

38

39

40

41

42

43

44

45

46

47

48

49

50

51

52

53

54

55

56

57

58

59

60

http://mc.manuscriptcentral.com/tandf/jenmol 


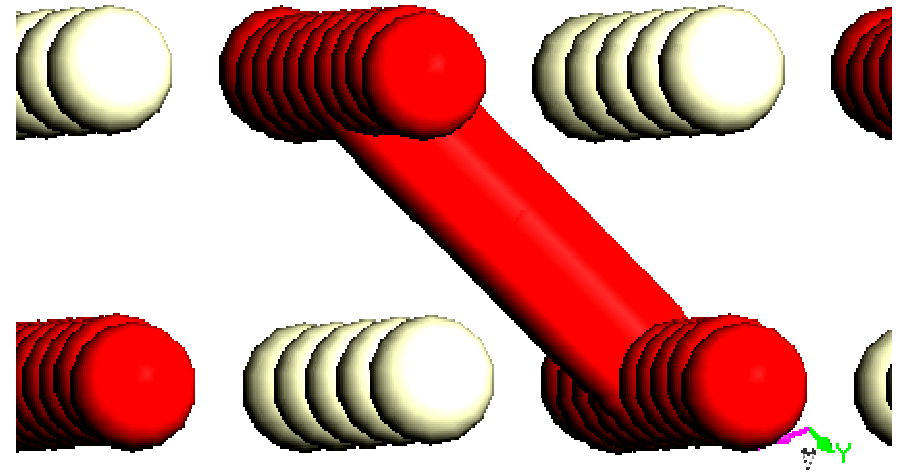

Figure 1: Oxygen Atom Migrating in The Middle of $\{111\}$ Surface Slab

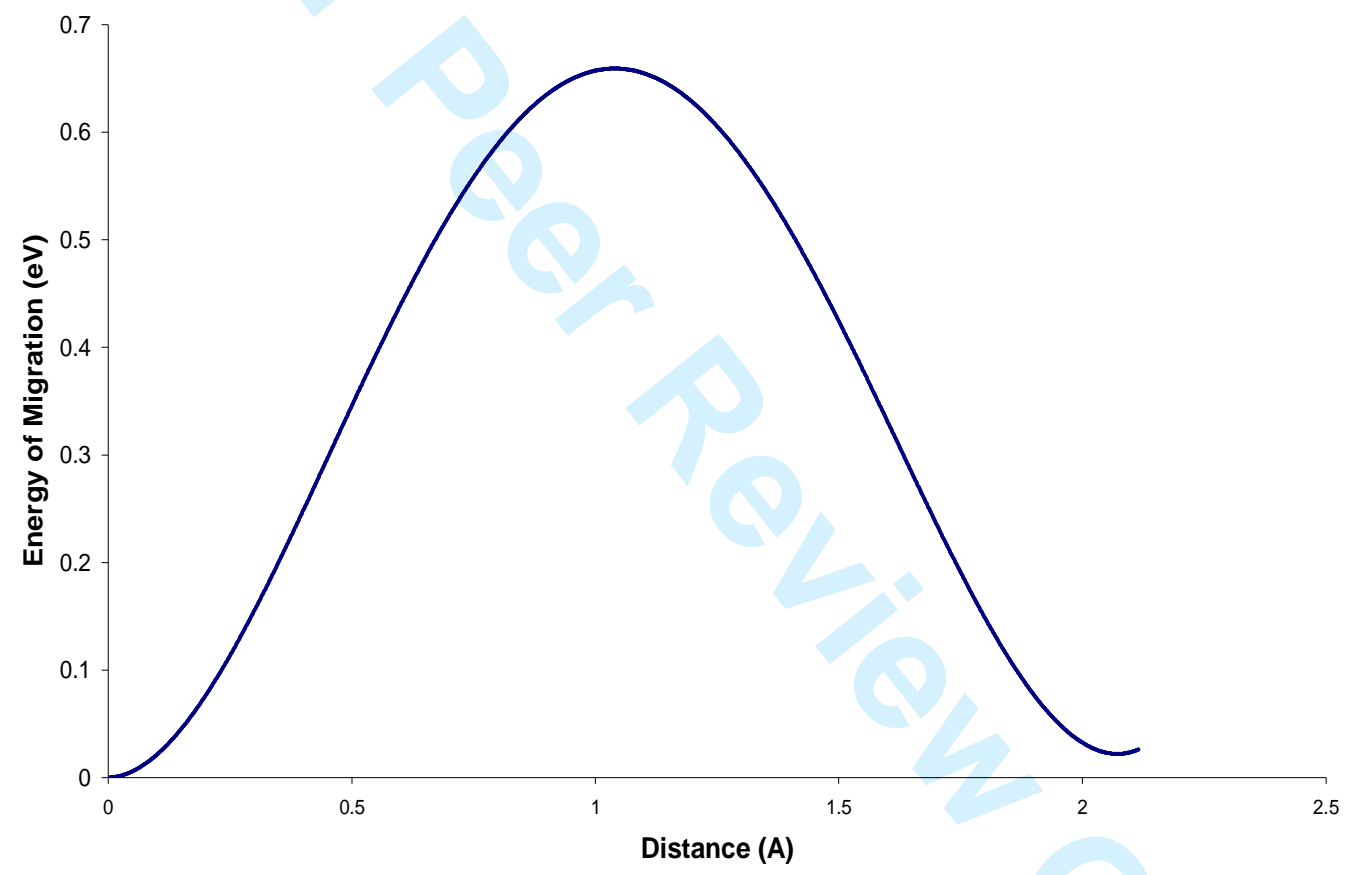

Figure 2: Energy Profile of Bulk Oxygen Migration 


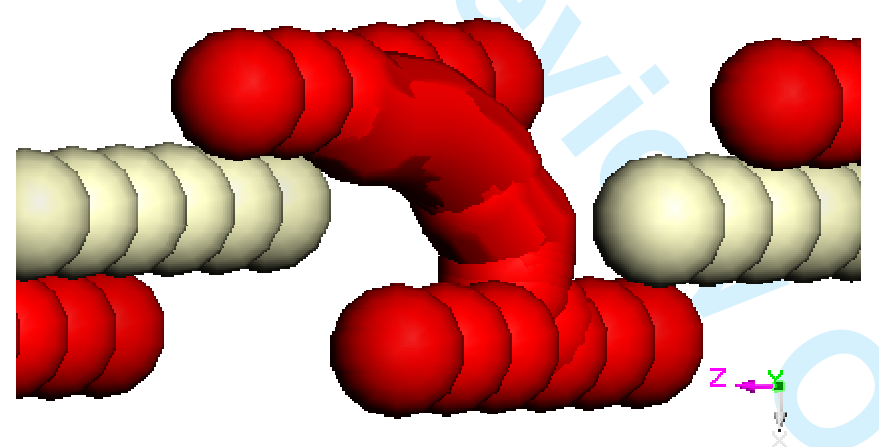

Figure 4: Oxygen Atom Migrating in the $\{111\}$ Surface 


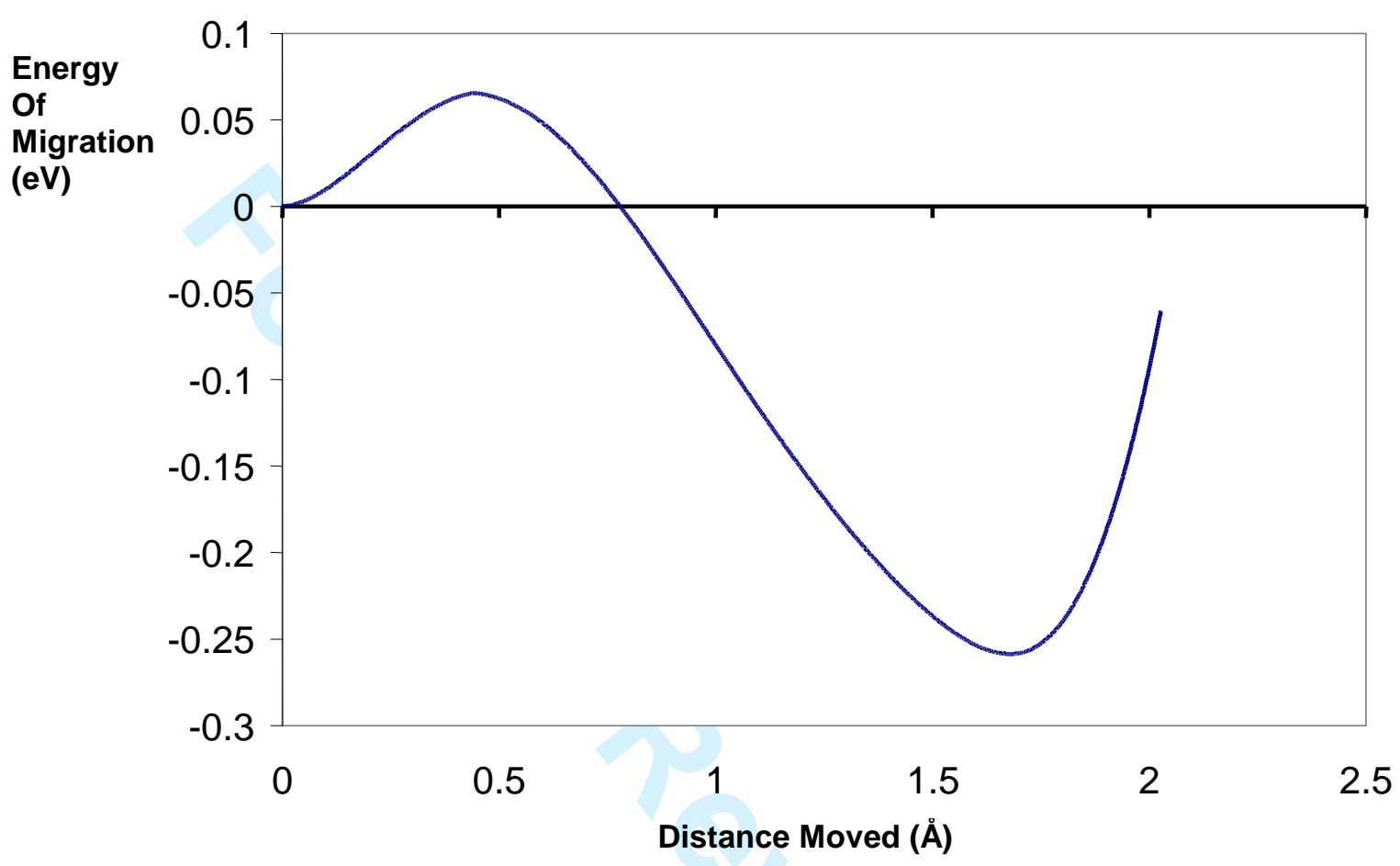

Figure 5: Energy Profile of Oxygen Migration in the $\{111\}$ Surface. 
1

2

3

4

5

6

7

8

9

10

11

12

13

14

15

16

17

18

19

20

21

22

23

24

25

26

27

28

29

30

31

32

33

34

35

36

37

38

39

40

41

42

43

44

45

46

47

48

49

50

51

52

53

54

55

56

57

58

59

60
Energy of
Migration

(eV)

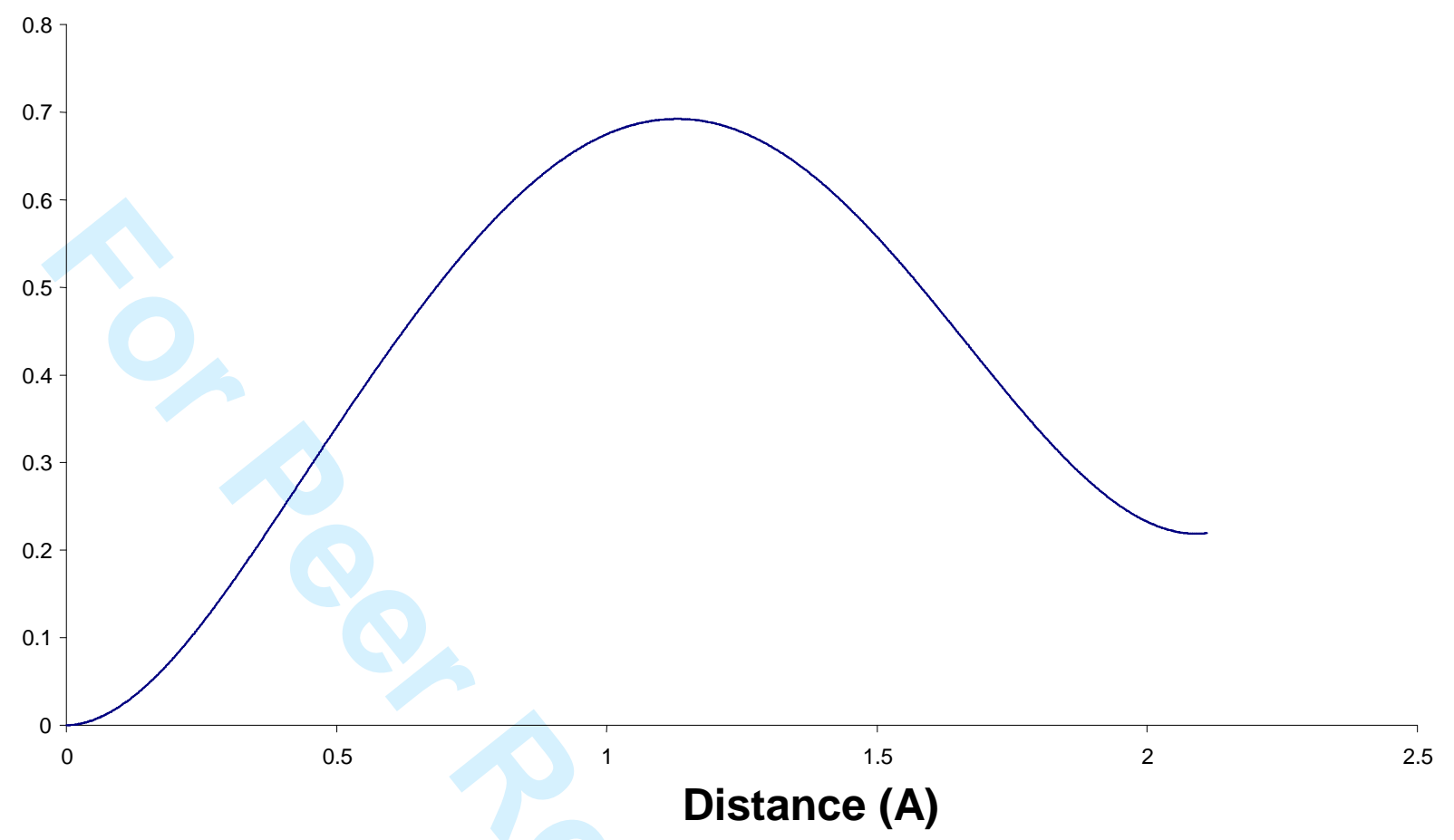

Figure 6: Energy Profile of Oxygen Migration from Bulk to Just Under the $\{111\}$ Surface 


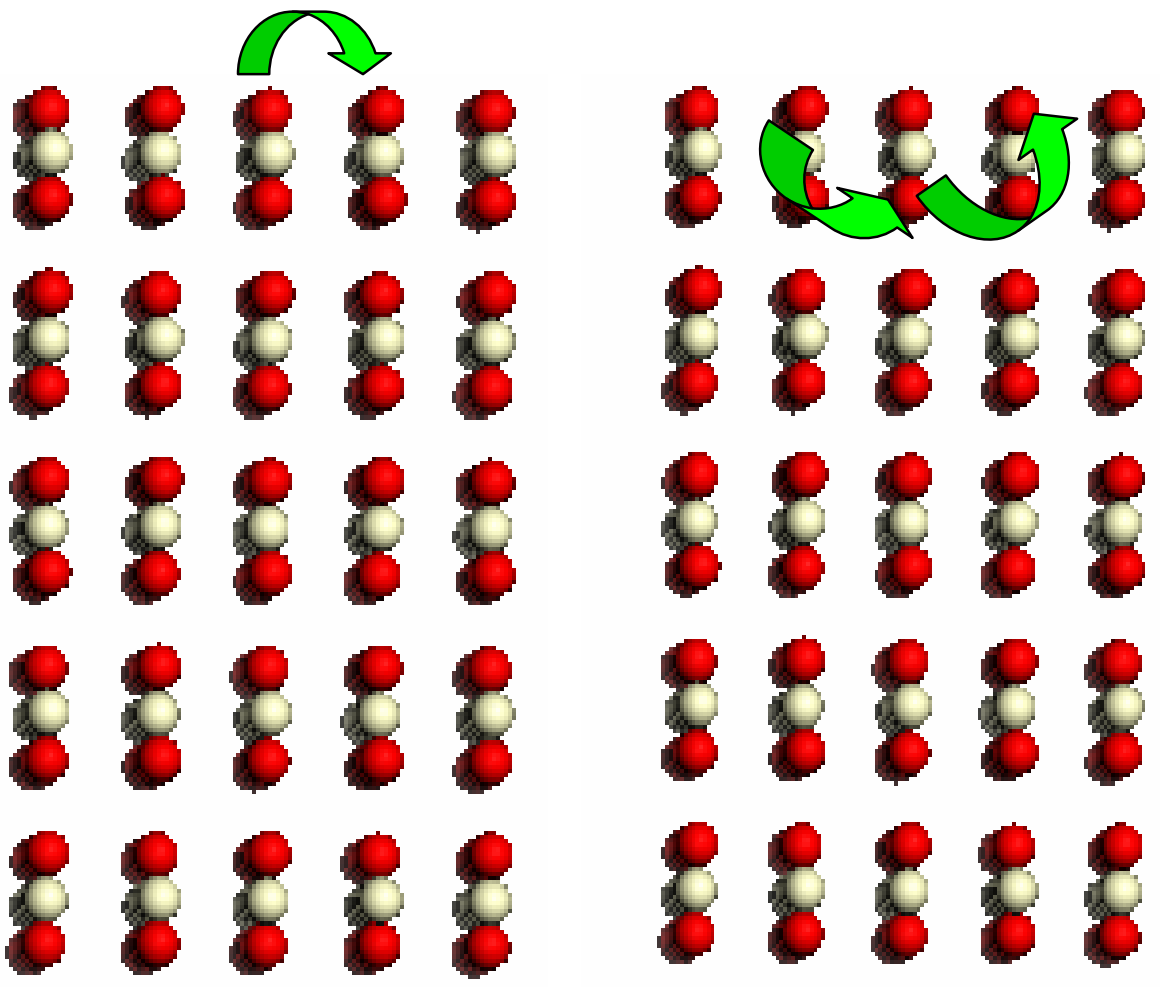

Figure 7 (a) Schematic of oxygen hopping across the surface. $\Delta \mathrm{G} \sim 2.5 \mathrm{eV}$

Figure 7 (b) Schematic of oxygen moving through the surface. $\Delta \mathrm{G} \sim 0.3 \mathrm{eV}$

@ $\mathbf{T}=\mathbf{3 0 0} \mathbf{K}$

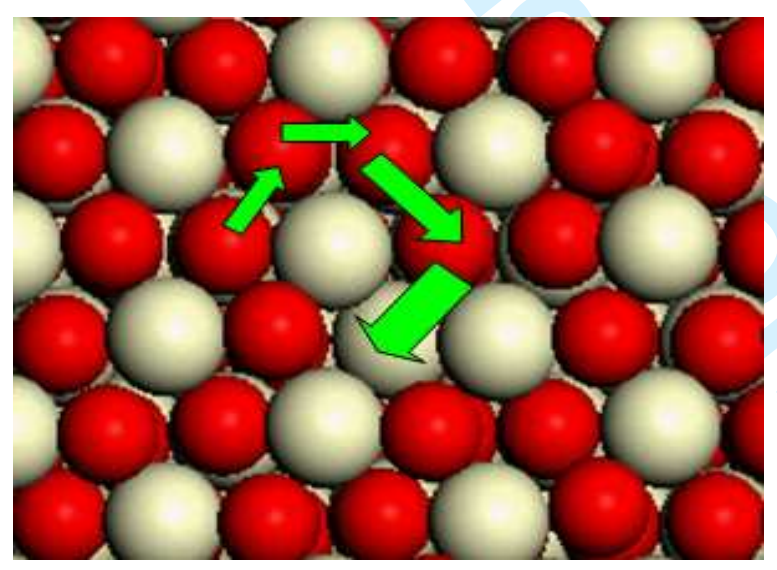

Figure 8: Pathway of Concerted Mechanism 


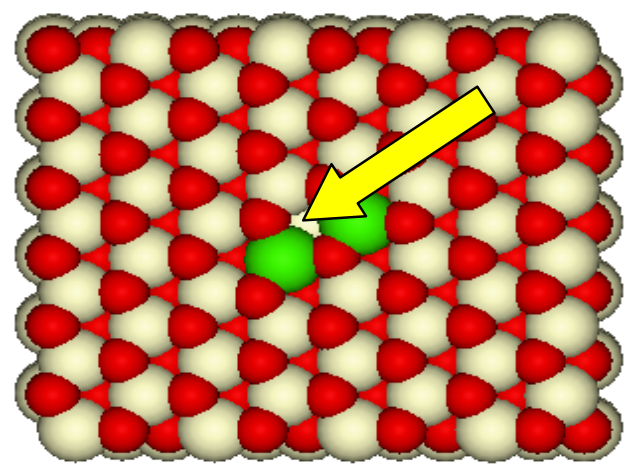

Figure 9a: Before migration and with Surface Vacancy in place. Yellow arrow points to Oxygen that is to be constrained to migrate.

Figure 9b: After Oxygen atom, originally under the surface between $\mathrm{Ce}^{3+}$ ions, has migrated to the surface leaving vacancy just

under the surface and between $\mathrm{Ce}^{3+}$ ions. Green $=\mathrm{Ce}^{3+}$, White $=\mathrm{Ce}^{4+}, \mathrm{Red}=\mathrm{O}^{2-}$ The resultant subsurface vacancy is highlighted by the blue arrow.

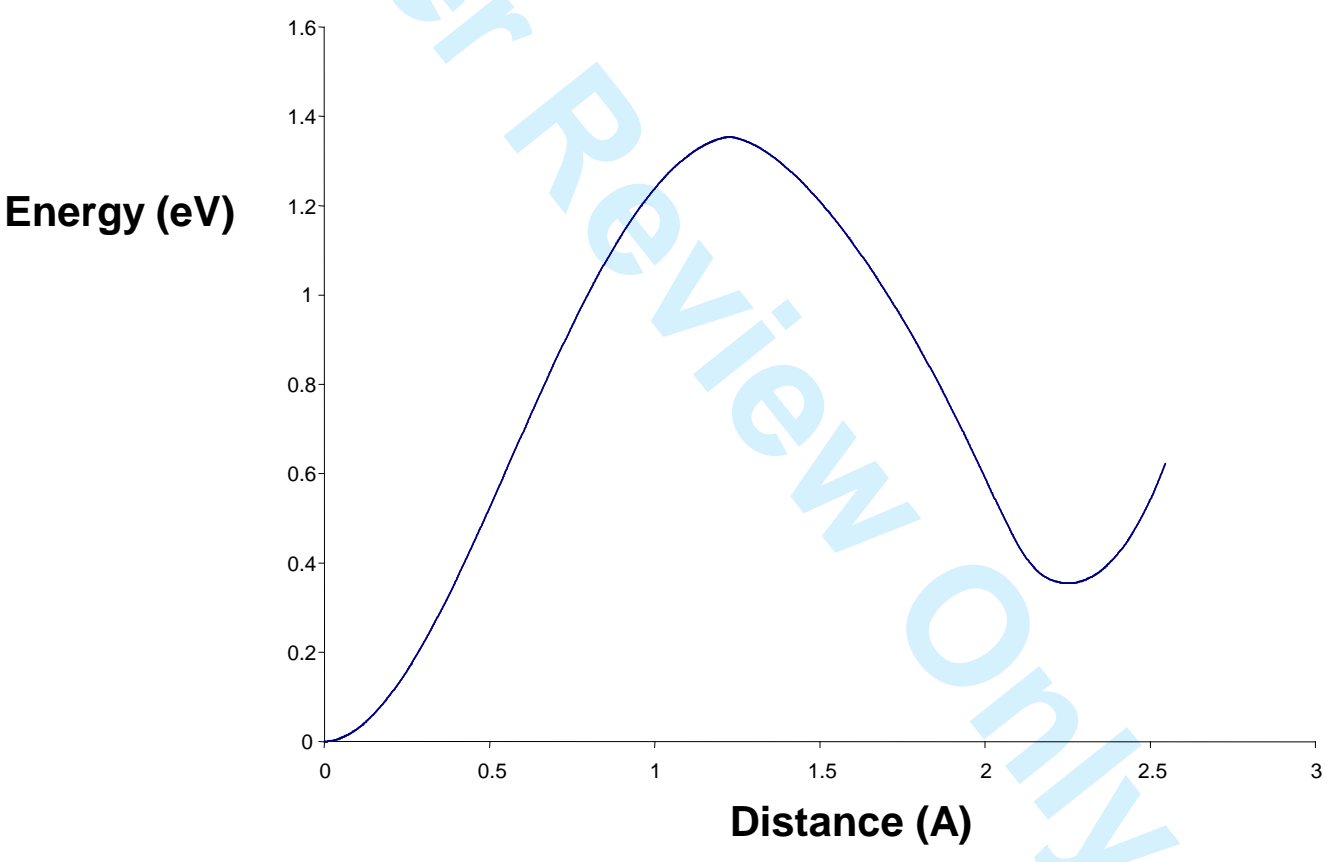

Figure 10: Energy Profile of Oxygen atom migration between $\mathrm{Ce}^{3+}$ ions onto the Surface 

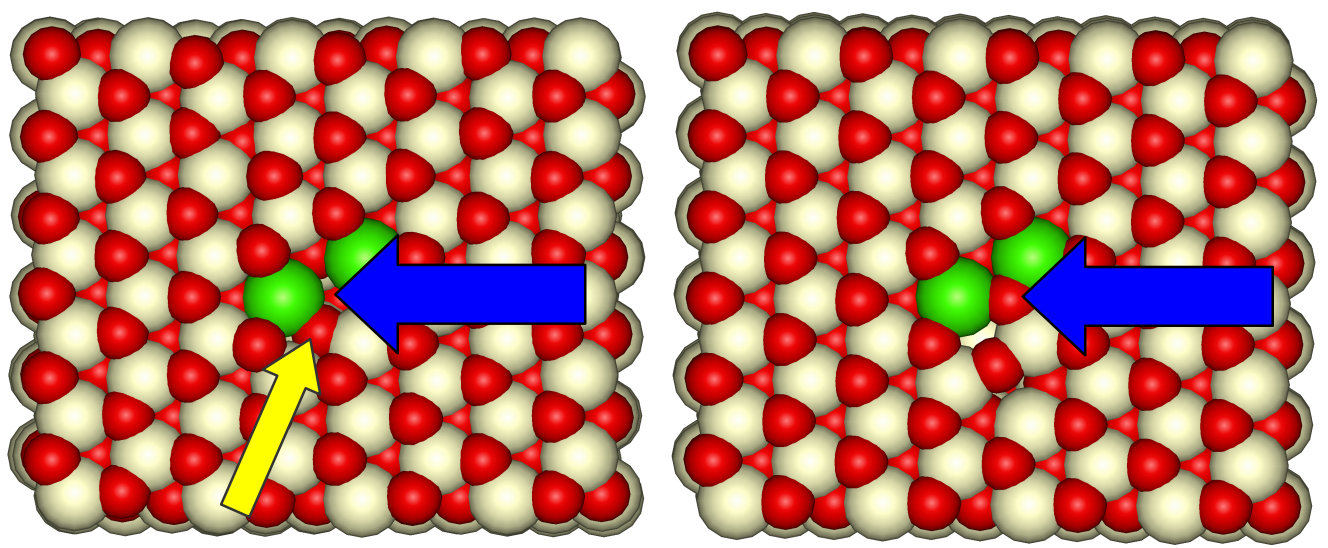

Figure 11a: Before migration and with Surface Vacancy in place (blue arrow). Yellow Arrow points to Oxygen atom to migrate from just beneath the surface.

Figure 11b: After. Oxygen atom, originally under the surface and below the vacancy, has migrated to the surface leaving vacancy just under the surface and below the left $\mathrm{Ce}^{3+}$ ion. Green $=\mathrm{Ce}^{3+}$, White $=\mathrm{Ce}^{4+}, \mathrm{Red}=\mathrm{O}^{2-}$

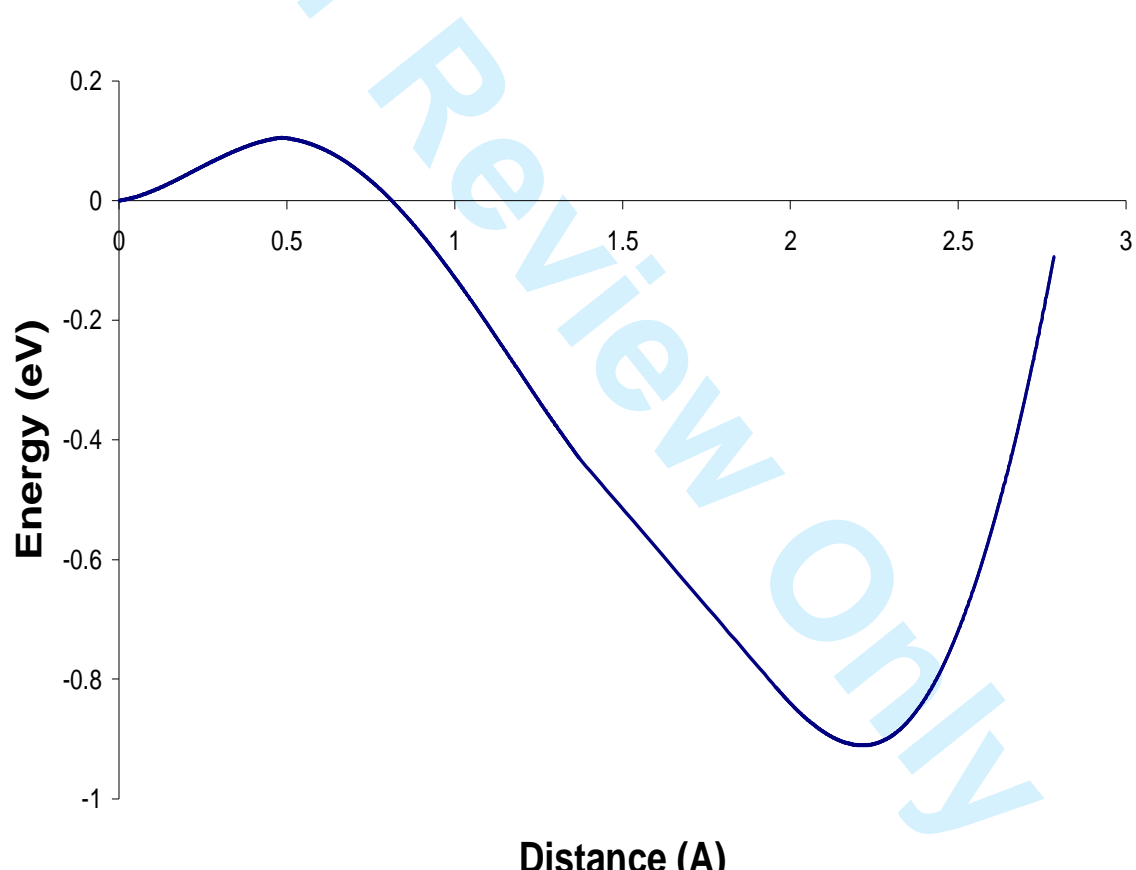

Figure 12: Energy Profile of Oxygen atom migration From under the Left $\mathrm{Hand}^{\mathrm{Ce}^{3+}}$ ion into the Surface Vacancy between Compensating $\mathrm{Ce}^{3+}$ ions 


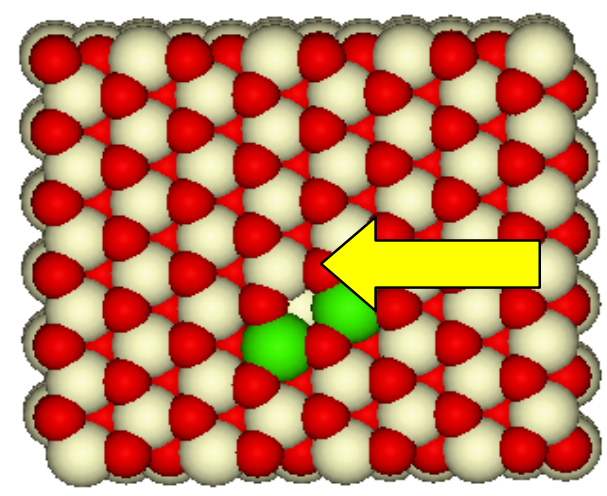

Figure 13a Before migration and with Surface Vacancy in place.

Figure 13b: After. Oxygen atom, originally under the surface between $\mathrm{Ce}^{3+}$ ions, has migrated to the surface leaving vacancy just

under the surface and between $\mathrm{Ce}^{3+}$ ions. Green $=\mathrm{Ce}^{3+}$, White $=\mathrm{Ce}^{4+}, \mathrm{Red}=\mathrm{O}^{2-}$

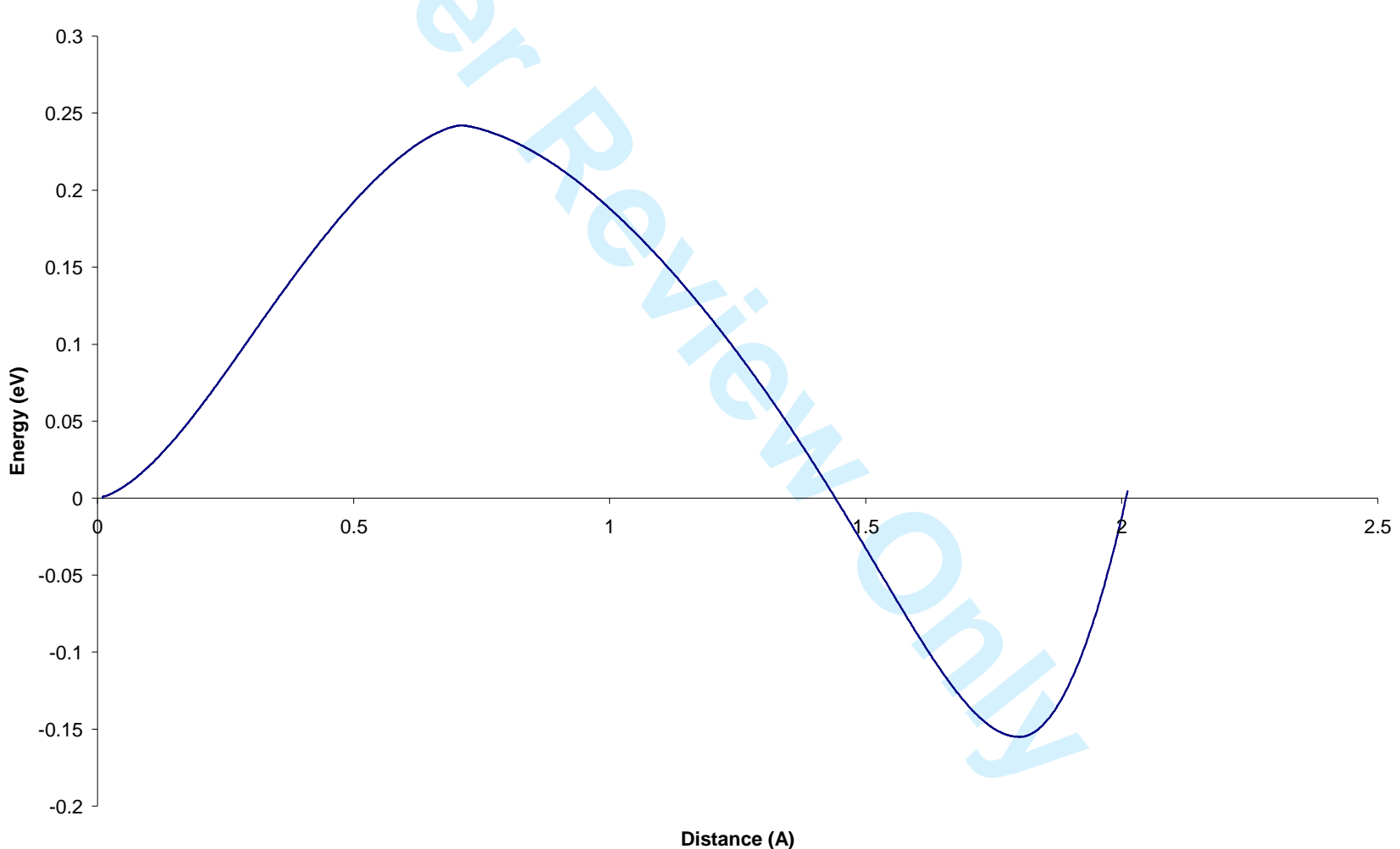

Figure 14: Energy Profile of Oxygen atom migration Away from $\mathrm{Ce}^{3+}$ ions onto the Surface 


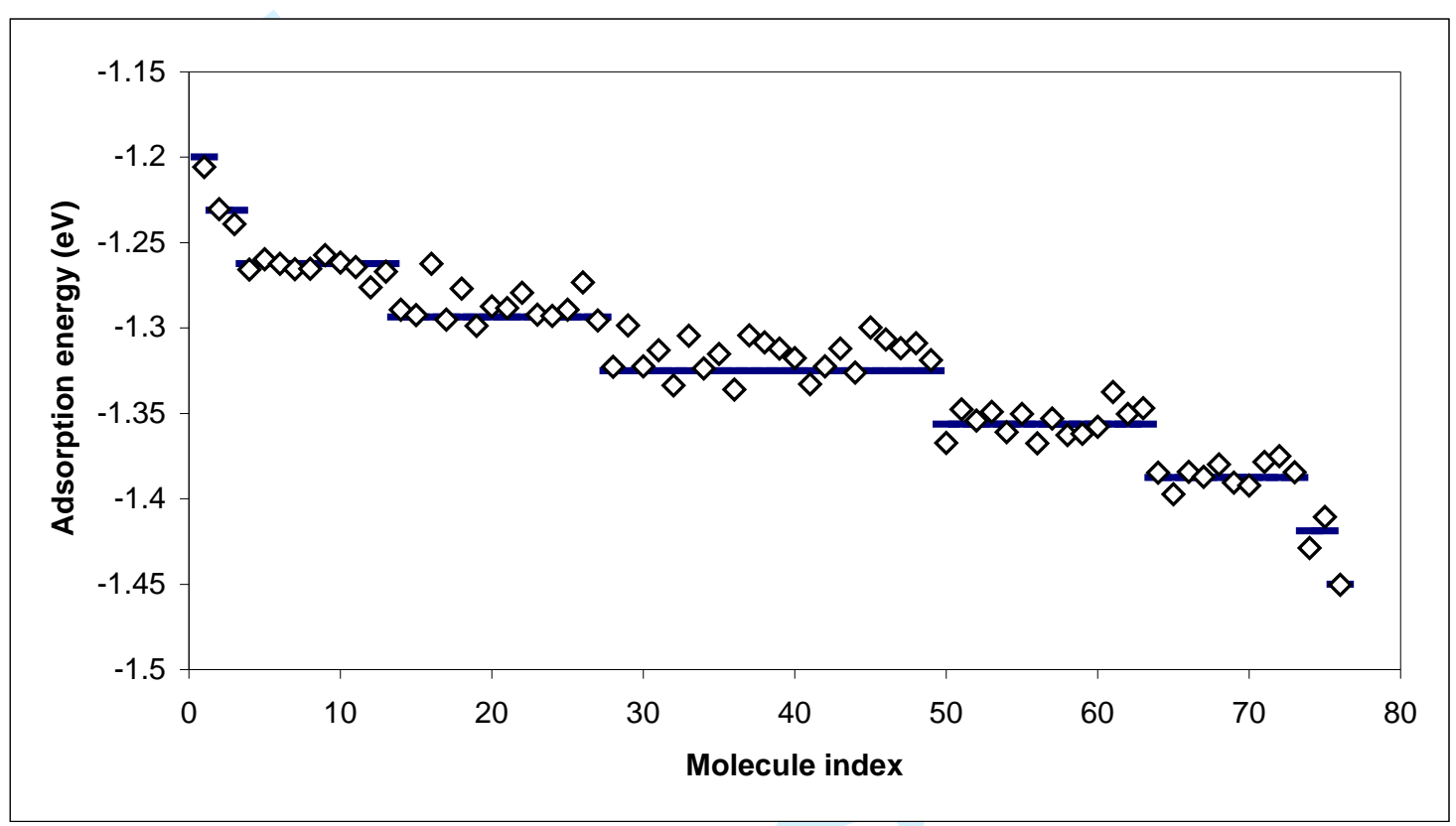

Figure 15: Adsorption energy of the 76 PCDD

http://mc.manuscriptcentral.com/tandf/jenmol 
Figure 16: Adsorption position of a PCDD congener. The surface is parallel to the page. The shaded atoms represent the lower layers. Surface: Si Large light grey, $O$ small dark grey. Molecule: $H$ white, $\mathrm{Cl}$ light grey, $\mathrm{C}$ grey, $\mathrm{O}$ dark grey 

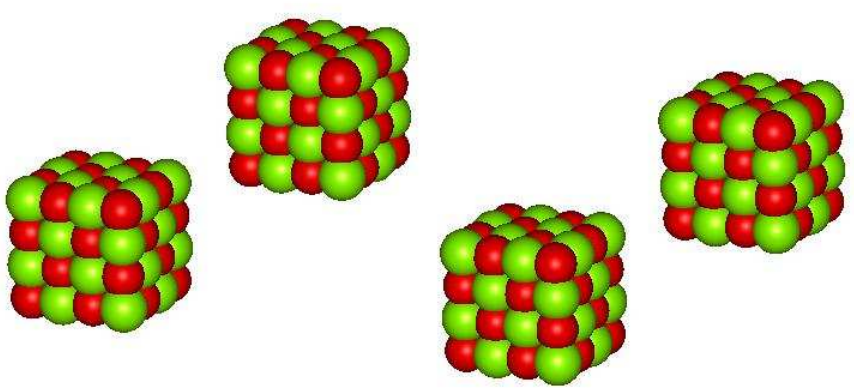

Figure 17: Initial configuration of eight $\mathrm{MgO}$ nanoparticles before aggregation

\section{Potential energy against time of an MD simulation of $8 \mathrm{MgO}$ nanoparticles}

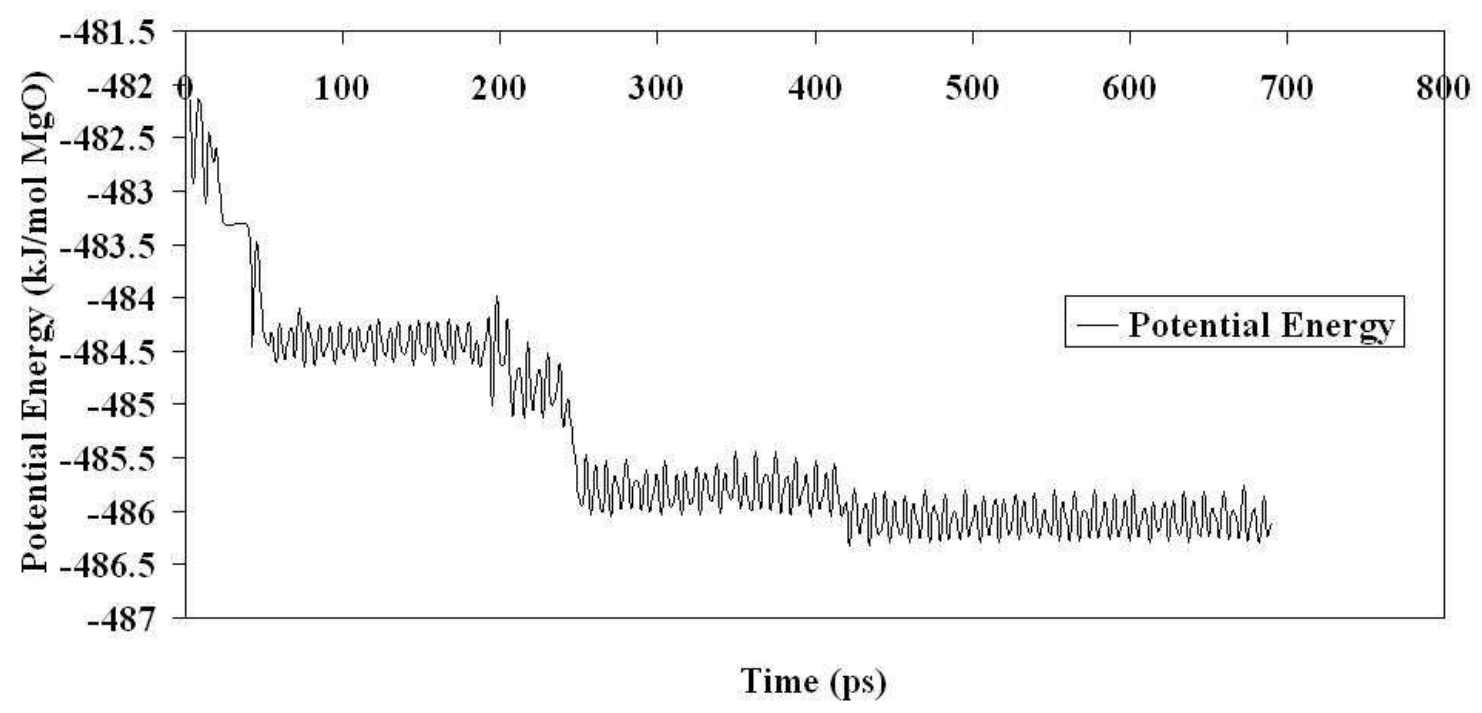

Figure 18: Potential energy against time of an MD simulation of $8 \mathrm{MgO}$ nanoparticles 
Figure 19: A snapshot of the MD simulation at 30ps for the MgO system

Figure20: A snapshot of the MD simulation at 60ps for the MgO system 
Figure 21: A snapshot of the MD simulation at 220ps for the MgO system
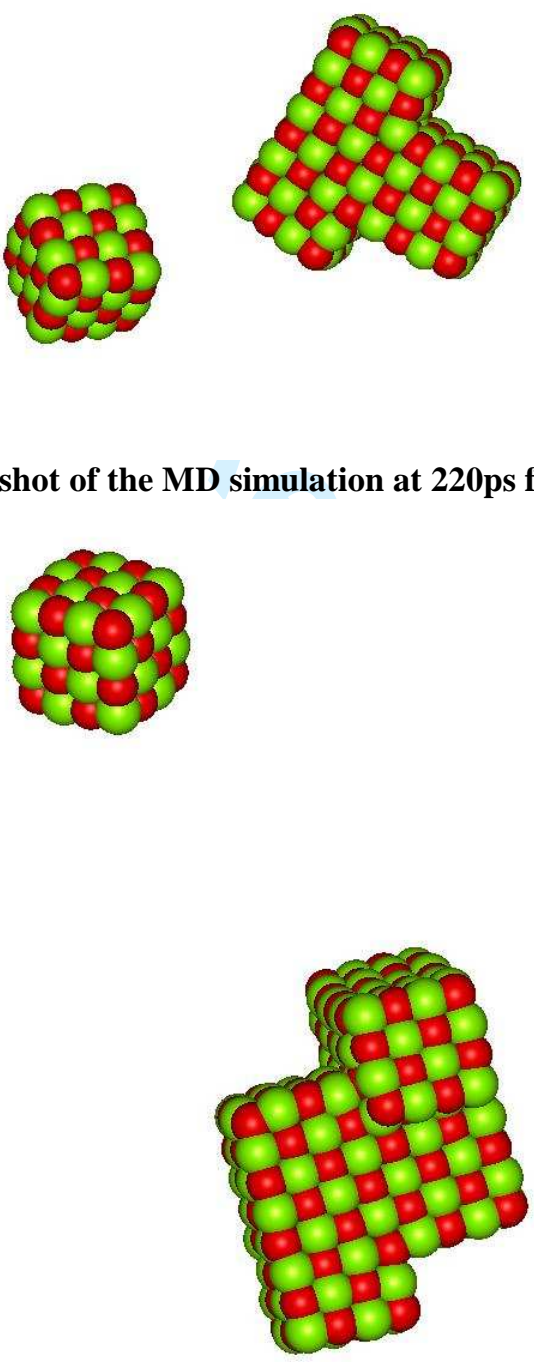

Figure 22: A snapshot of the MD simulation at 275ps for the MgO system 


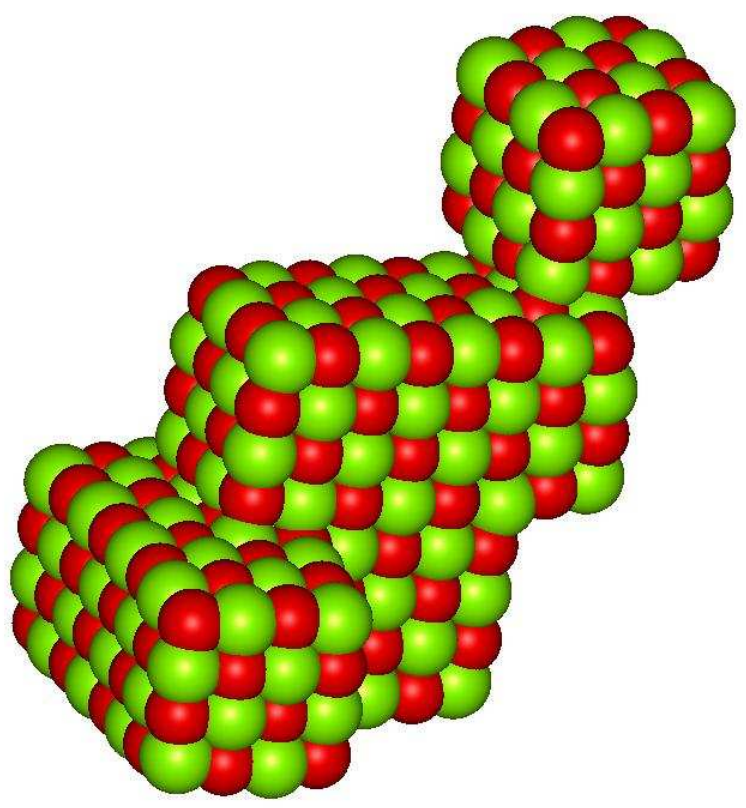

Figure 23: Final configuration of the aggregated MgO nanoparticles 


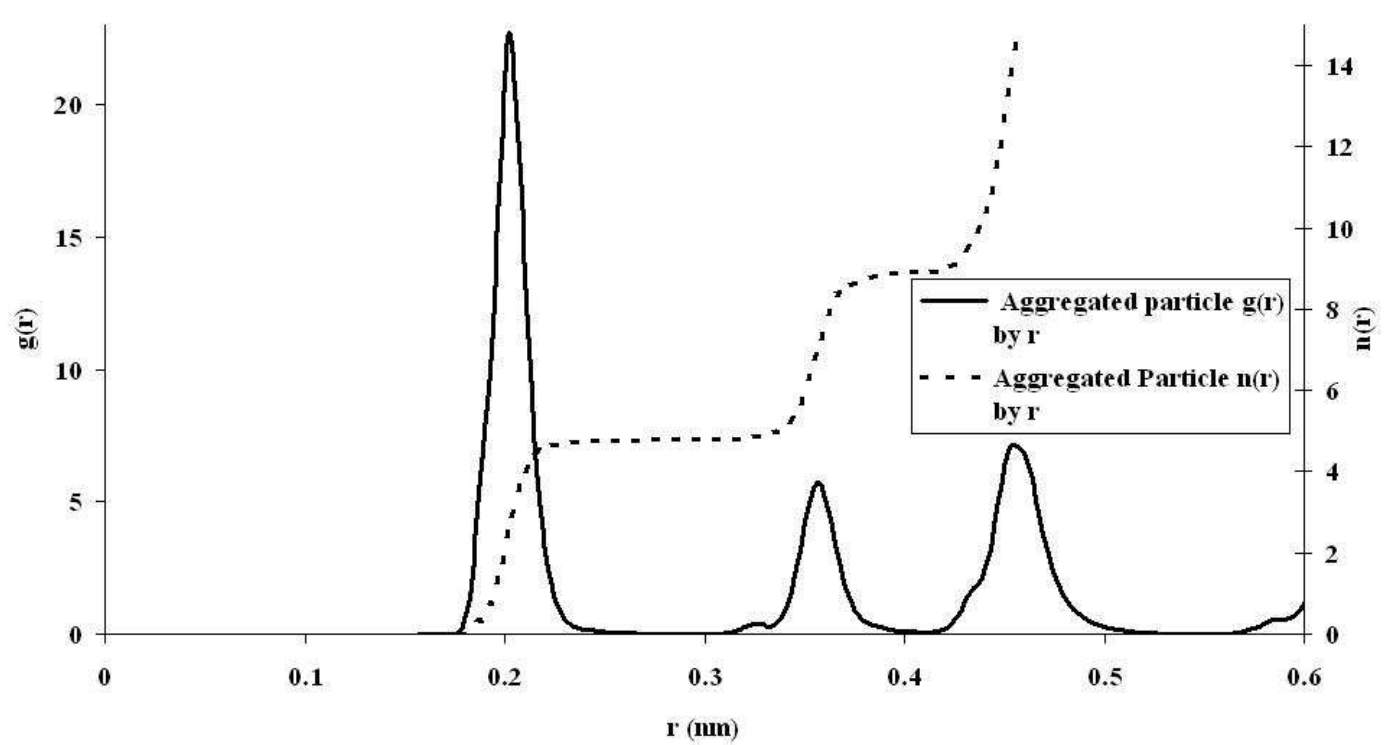

Figure 8b: Mg-O RDF of Bulk Magnesiun Oxide

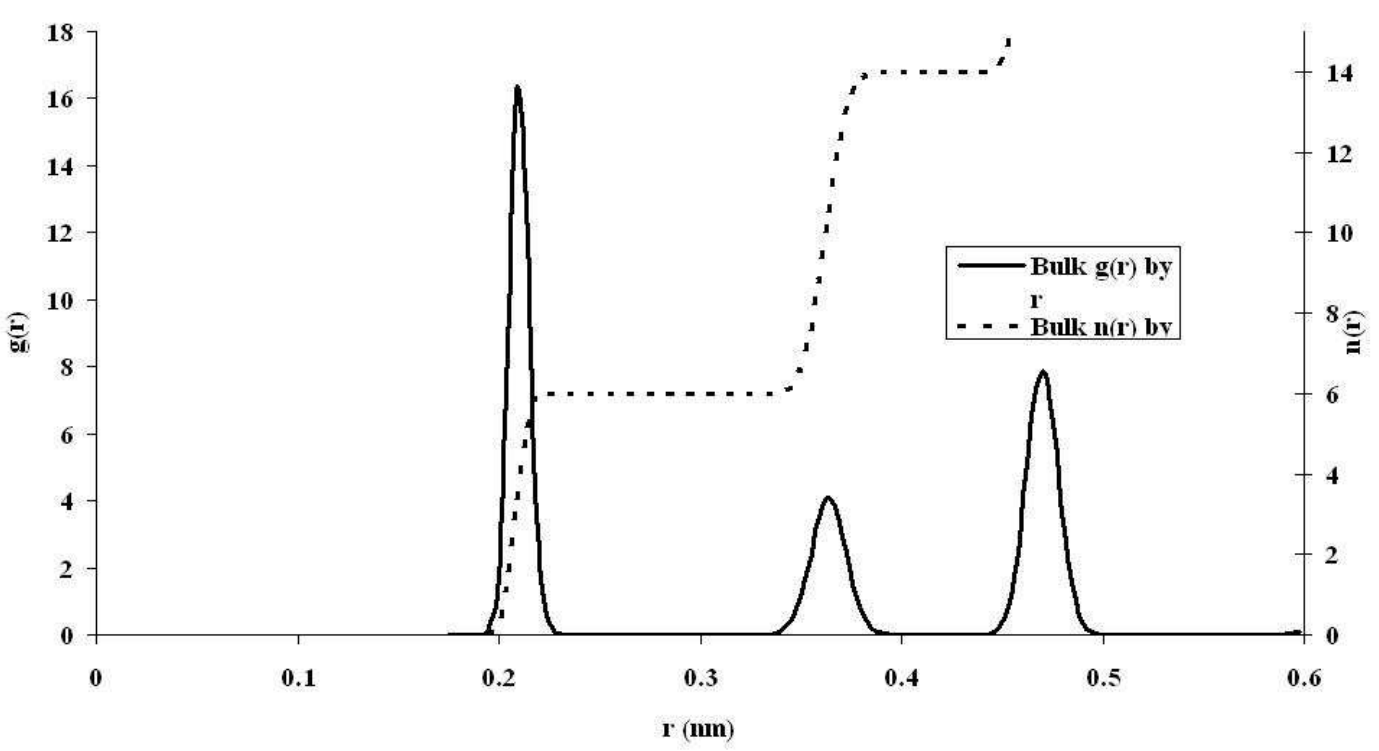

Figure 24: RDF of MgO (a) aggregated nanoparticle and (b) Bulk 
Figure 25: Initial configuration of calcite nanoparticles for MD simulation

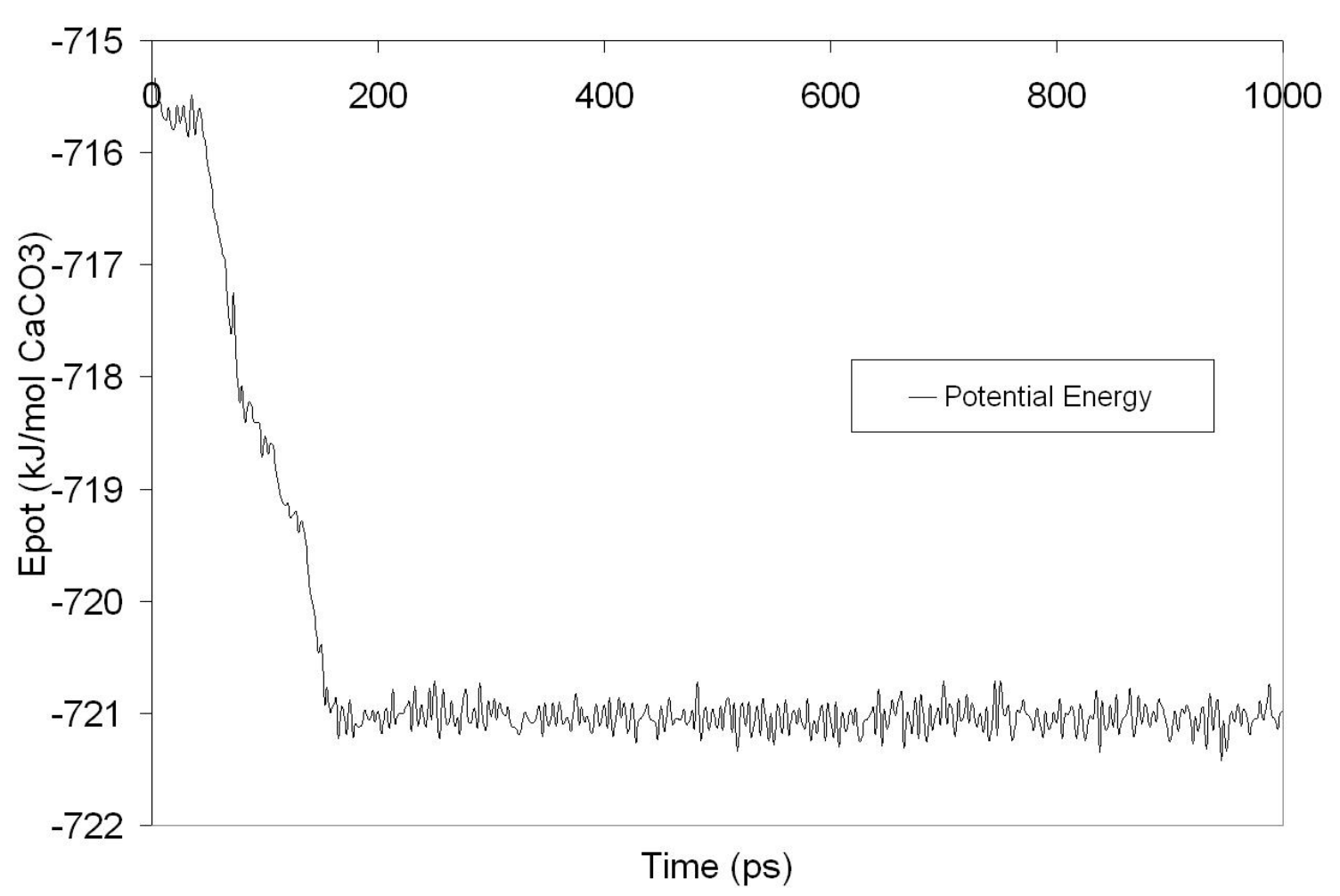

Figure 26: Potential energy against time of an MD simulation of 8 calcite nanoparticles 
Figure 27: A snapshot of the MD simulation at 50ps for the calcite 1.6nm system 


\section{Page 47 of 158}

1
2
3
4
5
6
7
8
9
10
11
12
13
14
15
16
17
18
19
20
21
22
23
24
25
26
27
28
29
30
31
32
33
34
35
36
37
38
39
40
41
42
43
44
45
46
47
48
49
50
51
52
53
54
55
56
57
58
59
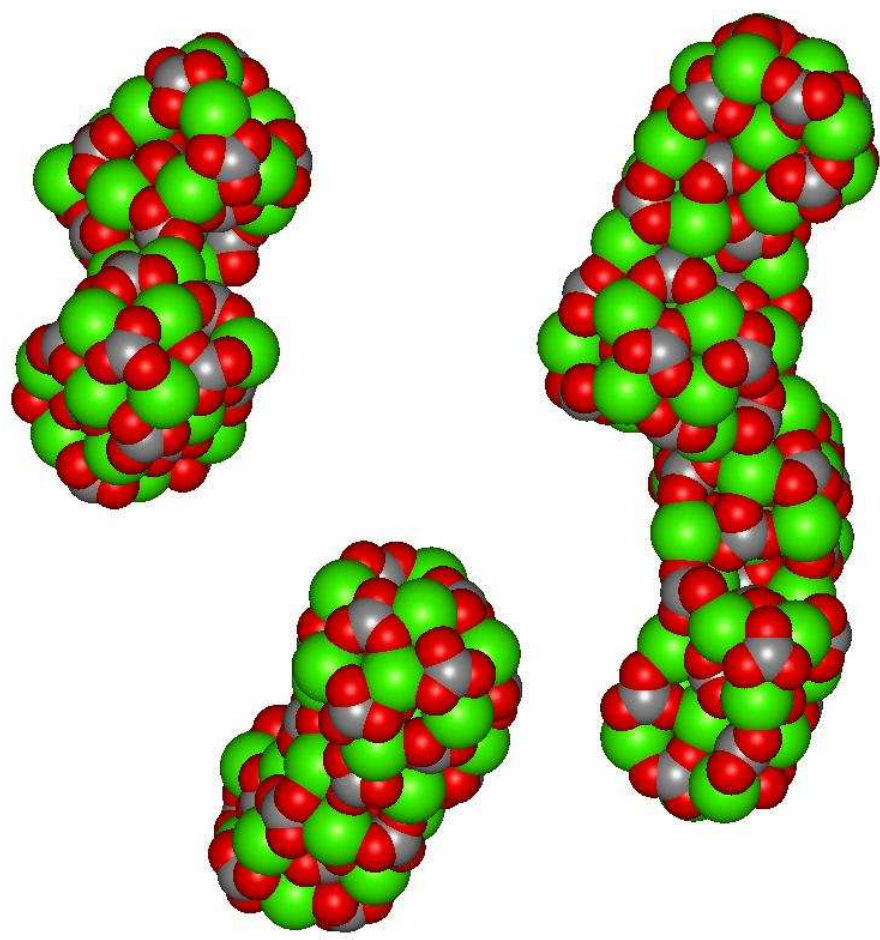

Figure 28: A snapshot of the MD simulation at 87ps for the calcite 1.6nm system 


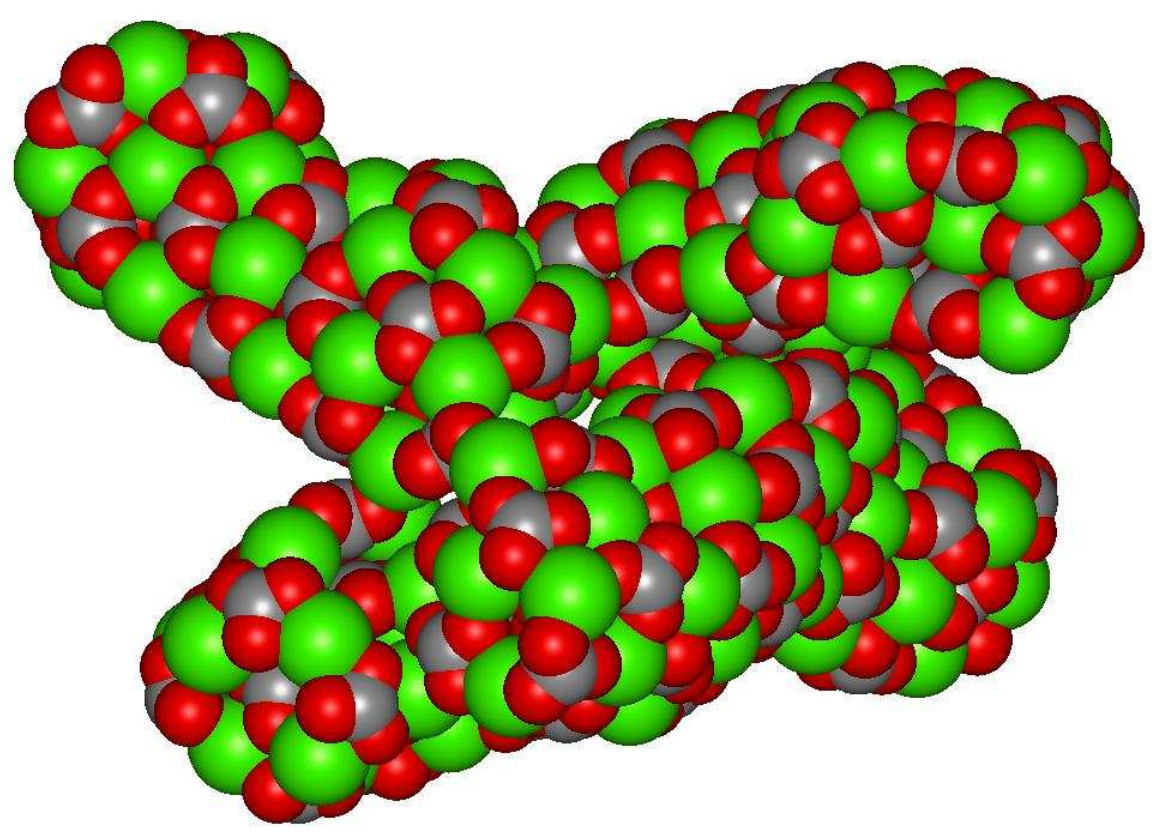

Figure 29: snapshot of the MD simulation at 140ps for the calcite 1.6nm system

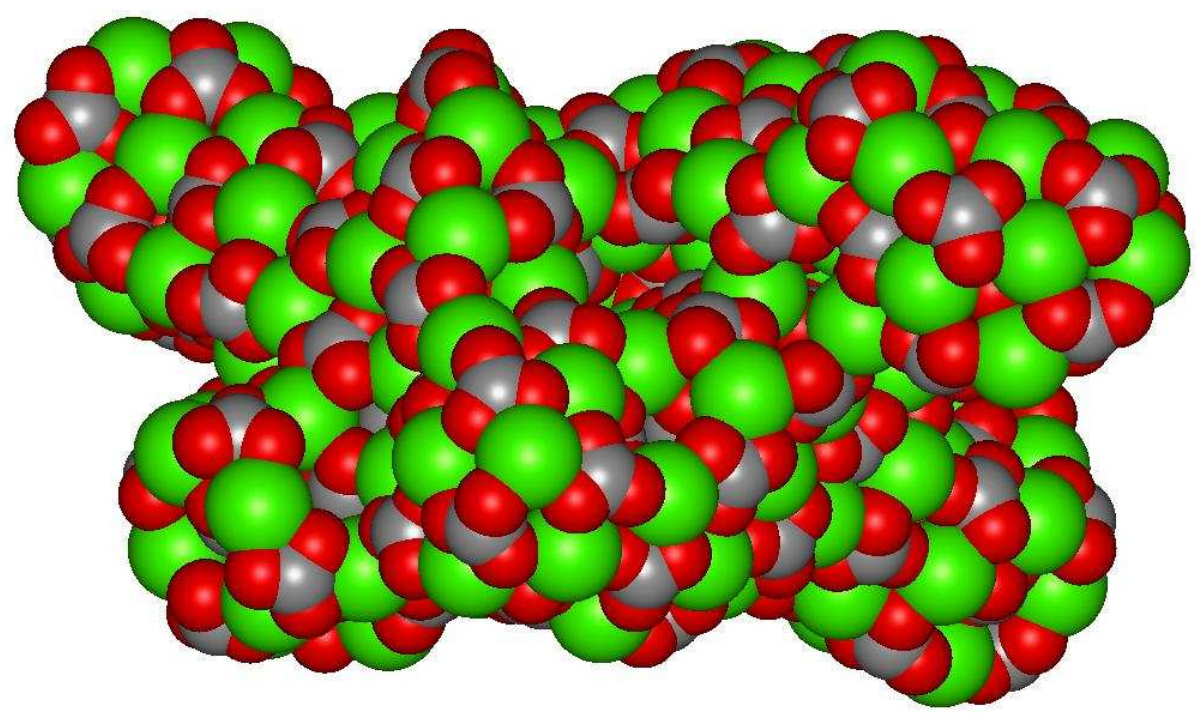

Figure 30: Final configuration of the aggregated calcite nanoparticles 
Figure 15a: Ca-O RDF of the Aggregated Calcite Nanoparticle

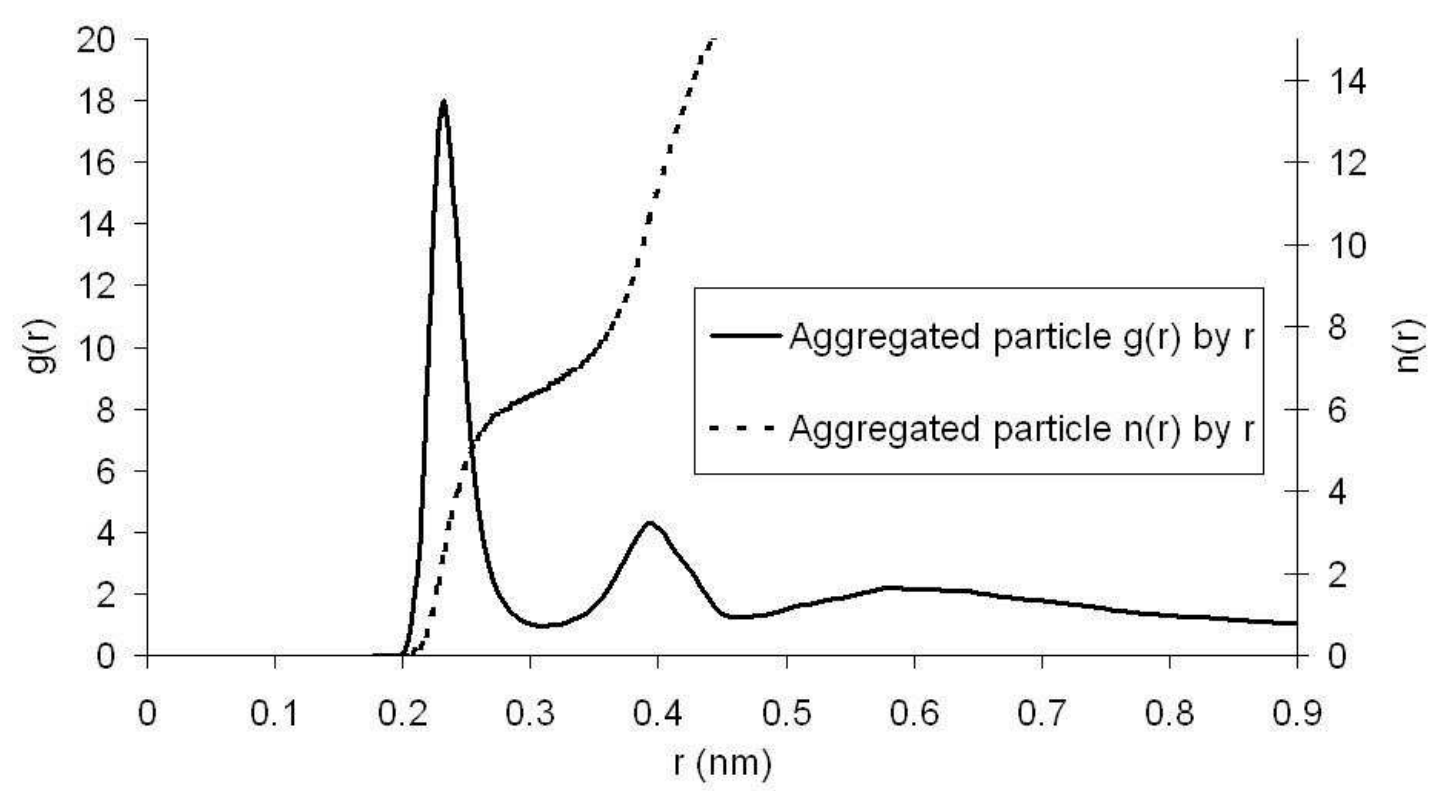

Figure 15b: Ca-O RDF in Bulk Calcite

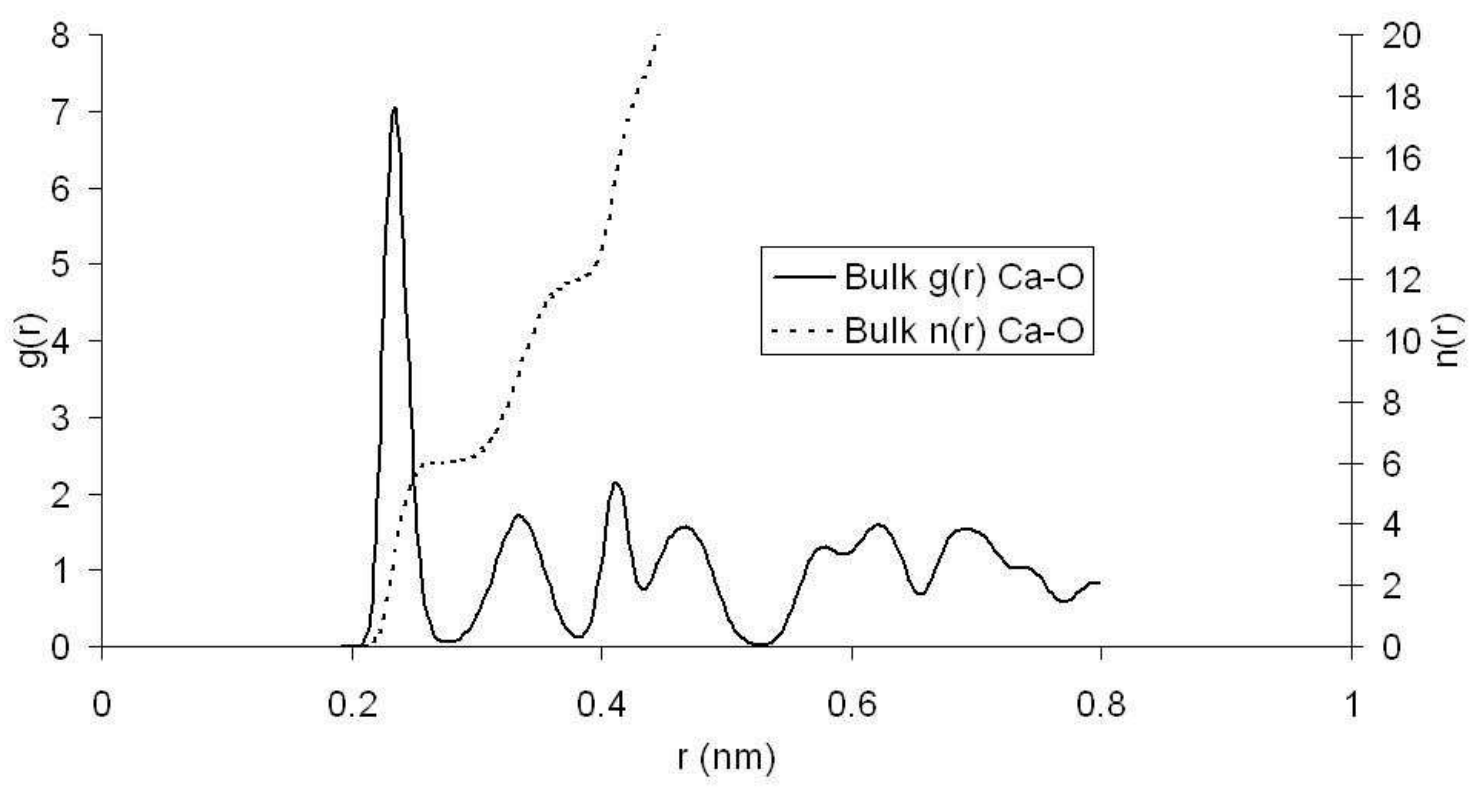

Figure 31: RDF of the (a) aggregated calcite nanoparticles and (b) bulk calcite 


\begin{tabular}{lc}
\hline \hline \multicolumn{1}{c}{ Specie } & Charge \\
\hline $\mathrm{H}$ & .20 \\
$\mathrm{Cl}$ & -.03 \\
$\mathrm{O}$ & -.50 \\
$\mathrm{C}(\mathrm{H}$ bonded $)$ & -.20 \\
$\mathrm{C}(\mathrm{Cl}$ bonded $)$ & .03 \\
$\mathrm{C}(\mathrm{O}$ bonded $)$ & .25 \\
\hline \hline
\end{tabular}

Table 1: Charge model (in e)

1

2

3

4

5

6

9

10

11

12

13

14

15

16

17

18

19

20

21

22

23

24

25

26

27

28

29

30

31

32

33

34

35

36

37

38

39

40

41

42

43

44

45

46

47

48

49

50

51

52

53

54

55

56

57

58

59

60

http://mc.manuscriptcentral.com/tandf/jenmol 


\section{Application of Molecular Dynamics DL_POLY Codes to}

\section{Interfaces of Inorganic Materials}

Paul Martin, Dino Spagnoli, Arnaud Marmier, Stephen C. Parker.

Department of Chemistry, University of Bath, Claverton Down, Bath, UK

BA2 7AY. E-mail: s.c.parker@ bath.ac.uk: Fax: +44 1225 386231, Tel: +44

1225386505

Dr. Dean C. Sayle Defence Academy of the United Kingdom, Cranfield

University Shrivenham, Swindon United Kingdom SN6 8LA. E-mail:

d.c.sayle@ cranfield.ac.uk Fax:+44 (0)1793 785772 , Tel:+44 (0) 1793

785264

Dr. Graeme Watson School of Chemistry, University of Dublin, Trinity

College, Dublin 2. E-mail: watsong@tcd.ie Fax:+353 (0)1 6712826 Tel:

+353(0)1 6081357 .

Abstract: Three recent applications of the DL_POLY molecular dynamics code are described, which demonstrate the flexibility and viability of the code for extending our understanding of the structure, stability and reactivity of ceramics and minerals at the atomic level. The first is an investigation into differences in oxygen atom mobility in bulk and at the most stable $\{111\}$ surface of ceria. The results show enhanced surface transport but that it is via subsurface oxygen. Secondly, we investigate how polychlorodibenzo-pdioxins (PCDDs) molecules might adsorb on clay surfaces. The resulting adsorption energies show a clear relationship with chlorine content of the molecule. Finally, we apply DL_POLY to comparing the aggregation of magnesium oxide and calcium carbonate nanoparticles. We find that very small calcium 
carbonate nanoparticles are amorphous and their aggregation shows no preferred orientation in contrast to magnesium oxide, which remain highly crystalline and combine in a highly structural specific way.

\section{Introduction}

The aim of this paper is to describe our current work using DL_POLY to model the structures and energetics of inorganic solids, with particular emphasis on surfaces and nanostructures. We illustrate the scope of DL_POLY to modelling surfaces and interfaces by considering three recent examples. The first example describes our application of DL_POLY to explore the stability and transport of surface defects, oxygen vacancies on the most stable surface of ceria. Understanding the factors controlling the formation of surface defects is of importance for understanding surface properties. As it is the surface

Deleted:

Deleted: to

Deleted: Another component of chemical reactivity is understanding how molecules might adsorb onto surfaces. Here group [1] by applying DL_POLY to help our understanding of adsorption, processes. Nanoparticles are of great scientific interest as they are effectively a bridge between bulk materials and atomic or molecular structures. The interesting and sometimes unexpected properties of nanoparticles are partly due to their large number of surface sites aspects. The percentage of atoms at the surface of a material becomes significant as the size of that material approaches the nanoscale. Thus in our final example we describe recent work on studying the structure and aggregation of nanoparticles of two minerals.

\section{Molecular Dynamics at the Stable Low Index $\{111\}$ Surface of Ceria}

Deleted: section

Deleted: we describe

Deleted: properties 
Materials based on ceria $\left(\mathrm{CeO}_{2}\right)$ are used in the production and purification of hydrogen, the purification of exhaust gases in three-way automotive catalytic converters, and other catalytic applications [2-5]. These applications make use of the unusual properties of ceria, namely the ability to shift between its two oxidation states, Ce(III) and Ce(IV), and the high mobility of bulk oxygen species - properties that allow ceria to behave as an oxygen buffer. It is important therefore that we understand more fully how oxygen atoms move in the ceria surface, ${ }_{v_{-}}$In this study, we focused on studying transport at or near the $\{111\}$ surface of ceria, chosen because it is the most stable surface [8] and hence most prevalent.

The METADISE code (minimum energy techniques applied to dislocation, interface, and surface energies) [6] was used to construct the surface slabs for DL_POLY.

A slab of the $\{111\}$ surface was constructed to a thickness of $20.6 \AA$, which is sufficiently thick to allow realistic simulation of bulk ceria at the centre of the slab. The slab contains 996 atoms. $332 \mathrm{Ce}, 664$ O. We chose to use 3D periodicity for computational ease and hence introduced a $50 \AA$ vacuum gap. The Coulombic sums were calculated using Ewald summation precision of $10^{-5}$ which provides a straightforward way of specifying the number of reciprocal lattice vectors and indicates the precision to which the reciprocal component of the Ewald sum is converged.

The potential model used in this work was derived by Balducci et al (1997) [7] and is a modification of a potential derived by Sayle T.X.T. (1994) [8]. We included the shell model of Dick and Overhauser [9] to simulate ionic polarizability of the oxygen ions. In this model, the oxygen ion is represented as a core plus a shell coupled by a harmonic
Deleted: electrochemical

Deleted: this

Deleted: :

Deleted: just under the surface and in bulk

Deleted: which is calculated Deleted: to be

Deleted:

Deleted: is a potential from

Deleted: that

Deleted: [11] 
spring. The total charge is separated between the shell and the core. In the molecular dynamics simulations the shells were given a mass of 0.5 au.

In order to investigate oxygen atom mobility at the stable $\{111\}$ ceria surface a potential of mean force (PMF) method modification to DL_POLY $\left.\_10\right]$ has been used to simulate the pathway and variation in free energy of a single oxygen atom migrating in bulk ceria and also at surface.

The constrained atom is moved towards a vacancy at a constant velocity. The velocity chosen was $100 \mathrm{~m} / \mathrm{s}$, which is fast enough to obtain good statistics in sufficient cpu time but slow enough to allow the neighbouring ions to relax while the atom is migrating. $\underline{\text { Slower velocities did not make a significant difference to the energy profile. }}$

$$
\Delta A(z)=A(z)-A\left(z_{0}\right)=\int_{z_{0}}^{z} f_{z} d z
$$

$f_{\mathrm{z}}$ : average force in direction of vacancy.

The constrained atom is given a target vector, which is the co-ordinates of the lattice vacancy position. However, the constrained atom is free to move perpendicular to the target vector. The average force of the constrained atom along the target vector is recorded at the end of each time-step. Integration of the force with respect to distance yields the free energy, shown in equation (1), $\Delta A(z)$ is the free energy change of migration, $A(z)$ is the free energy at position $z$ and $A\left(z_{0}\right)$ is the free energy at the initial position. 
Thus the free energy of atom migration can be calculated at various temperatures. Additionally, MD simulation can be run just above zero Kelvin to enable the identification of the pathway and energy change neglecting temperature. This allows for simple (or an initial) analysis of the migration process, without involving complications of nearby atoms excessively hindering the constrained atom during its trajectory. In doing this, entropy effects are ignored, and therefore the energy of migration rather than the free energy of migration, is in fact calculated.

Migration of a single oxygen atom was investigated at the centre of the slab, which represents bulk ceria; just beneath the surface, with an oxygen migrating between bulk and a vacancy position just under the surface; and at the surface, where oxygen migration is simulated between the layer of oxygen atoms just beneath the surface and the surface layer, and also the migration from a surface position to a near surface vacancy.

\section{Oxygen Migration}

The first PMF simulations were carried out on the diffusion of oxygen in the bulk of the slab. We initially assumed that a vacancy was formed without a localised charge compensation, which we envisage as modelling the transport of the unbound oxygen vacancy. The slab was initially run at $300 \mathrm{~K}$ using the NVT ensemble, and the migration path plotted. The results show that the pathway between oxygen sites is linear (see Figure $\underline{1)}$ and that the energy of migration is $0.66 \mathrm{eV}$ with the transition state half way between the oxygen sites, see Figure 2. The activation energy agrees very well with the work of Balducci et al.(1997) [11] who calculated $0.65 \mathrm{eV}$ using a series of static energy

\begin{tabular}{|l|}
\hline Deleted: Figure 1 shows \\
\hline Deleted: was \\
\hline Deleted: energy profile in figure \\
2 shows that the maximum is the \\
\hline Deleted: wa \\
\hline Deleted: and that \\
\hline Deleted: the position was \\
\hline Deleted: $[9]$ \\
\hline
\end{tabular}
calculations. 
We can check that the location of the transition state is exactly halfway between the sites by plotting the average force of the constrained atom in the direction of travel in Figure 3. The force passes through zero at $1.05 \AA$ along a total distance of $2.07 \AA$.

We next modelled the trajectory for an oxygen atom migrating from the oxygen layer just below the surface to a vacant site on the surface.

Figure 4 shows the pathway. The first point to notice is the trajectory is now curved.

The energy plot in Figures 5 shows also a marked asymmetry. The transition state in this Deleted: case is very close to the surface $\sim 0.5 \AA$. Furthermore, Figure 5 shows that the activation energy for the oxygen migration from the surface to the level just beneath is considerably smaller ie. $0.06 \mathrm{eV}$. Interestingly, a greater activation energy, $0.3 \mathrm{eV}$, is required to activate the return of a subsurface oxygen directly to the $\{111\}$ surface from this layer beneath, which is still less than $0.66 \mathrm{eV}$, the bulk value. It also clearly demonstrates that the vacancy is more stable just below the surface.

The next stage was to examine the transport from the surface layers to the bulk. Figure 6 suggests the barrier is $0.67 \mathrm{eV}$, with the sub-surface site $0.2 \mathrm{eV}$ less stable.

Analysis of the trajectory shows that the migration path is not linear as in the bulk simulation, and that the position of the transition state is predicted to be slightly closer to the bulk layer than the sub-surface layer.

We also considered the migration of an oxygen atom across the top of the surface and surprisingly found that the activation energy was $2.5 \mathrm{eV}$. Thus Figure $7 \mathrm{a}$ and $7 \mathrm{~b}$ illustrates how a migrating oxygen atom would move down a layer just under the surface, and then would return to the surface layer leaving a vacancy in the sub-surface layer, 
which is the most stable site for an isolated vacancy, rather than transport across the surface, not unlike the recently proposed mechanism for surface transport by Allan and Harding [12]

We have seen more complex behaviour in some of the simulations, but still involving oxygen movement between the top two layers. Figure 8 shows the initial configuration of a concerted mechanism involving an oxygen atom in the surface layer constrained to move down into a vacancy position just under the surface. The initial constraint is shown by the largest green arrow in order. The constrained migration results in the formation of a vacancy in a less stable position on the surface because vacancies are more stable at a position just under the surface. The result is that, as the constrained oxygen leaves its lattice site to form a vacancy we find that the next oxygen atom fills the empty site and because the energy barriers are so low the next few oxygen atoms move in unison. The net result is that a sub-surface vacancy is formed but can be potentially some distance from the original vacancy.

The concerted motion does however highlight one problem, namely that the movement of the ions in the concerted mechanism causes a resistance to the movement of the constrained atom, thus the resulting free-energy change is the sum of the activation free energies of migration and the free energy for overcoming the drag-force. Thus care must be taken in interpreting the activation free energy.

Another important issue is the effect of charge compensation particularly when the charge compensating defects are bound to the oxygen vacancies. We consider them in the following section.

\section{Deleted:}

Deleted: from
Deleted: we find that

Deleted: $x$
Deleted: [49]

Deleted: . 
We treated the charge compensating defects as localised electrons on neighbouring cerium ions. Thus we first considered (Figure 9a) the oxygen atom migrating directly into a vacant site ${ }_{2}$ which is adjacent to two $\mathrm{Ce}^{3+}$ ions. The trajectory of migration takes the

Deleted: the first instance Deleted: is when

Deleted: es

Deleted: for the oxygen migration up and between the compensating ions compared to $0.06 \mathrm{eV}$ for similar migration without the involvement of compensating ions.

Another interesting result of this calculation is that the final configuration, with the vacancy just below the surface is calculated to be $0.4 \mathrm{eV}$ less stable. But note that the final oxygen vacancy position is not in the highly bound state (i.e.) just under the surface and adjacent to the charge compensating $\mathrm{Ce}^{3+}$ ions.

The second example (Figure 11a) considered is where an oxygen atom just beneath the surface is forced to migrate to a surface site that is adjacent to both the vacancy and a compensating $\mathrm{Ce}^{3+}$ ion, (i.e.) the oxygen vacancy moves from the top layer to the layer underneath. In this case, the activation energy of migration (Figure 12) is similar to that

Deleted: the Deleted:

Deleted: and to the side of

Deleted: $\mathbb{T}$

Deleted: instance for the un-charged compensated migration, (ie) $0.10 \mathrm{eV}$ cf. $0.06 \mathrm{eV}$.

The activation energy for the reverse process is approximately $1.0 \mathrm{eV}$, thus in common with the un-charged compensated migration, the vacancy is more stable below the surface but is very much more stabilised i.e., by about $0.9 \mathrm{eV}$.

Hence the energetics for the migration of an oxygen atom into a bound charge compensated vacancy cluster, when that oxygen atom approaches the vacancy from the 
side of the compensation, differ significantly from the case of migration between the compensating ions.

The final example considered (Figure 13a) involves the migration of an oxygen atom, which is originally under the surface between two adjacent $\mathrm{Ce}^{3+}$ ions, to a vacancy position set just to the side of this charge compensating pair of ions. The direction of migration is away from the charge compensation up into the vacancy which is considered here only to be partially bound because the vacancy is not symmetrically configured. Thus the final configuration leaves the vacancy in the highly bound state.

The plot in Figure 14 shows that the activation energies are still small, at $0.25 \mathrm{eV}$ for the forward and $0.4 \mathrm{eV}$ for the reverse.

However, activation migration energy is 4 times greater than the energy for the uncharged compensated case. It is 2.5 times greater than the migration into a bound charge compensated vacancy with a trajectory from the side, but is much smaller than the $1.35 \mathrm{eV}$ required for activation of migration up and between charge compensating ions into the fully bound vacancy.

The results show that energetically the vacancy prefers to be bound to the charge compensating defects in the subsurface oxygen site. Furthermore, the location of these charge compensating defects modify the activation energies and hence the mobility of oxygen near the surface. In the case of the movement along the site of the $\mathrm{Ce}^{3+}$ ions, the effect is significant and will have a large anisotropic effect on the atom transport.

The results therefore suggest that the migration of a defect cluster differs from isolated defects. Therefore, it is important to investigate the stability of different clusters. 
These simulation results suggest an important significance in the direction of migration around or between partially or fully bound charge compensated defects, in that it appears that oxygen atoms prefer to migrate up from the side of a bound compensated surface vacancy.

Lastly, it is important to note that a number of ab initio studies, for example Watson et al.[13-16l, have been performed on ceria. Comparison between the ab initio and potential based approaches used here, see for example [17l, have shown that the potentials are reliable.

\section{Organic molecules adsorbed on clay surface: DL_POLY as a high-throughput engine}

Organic pollutants and related molecules can pose a serious environmental threat to the global ecosystem. Amongst the most notorious are halogenated compounds substances used as pesticides or as by-products from other human activities. There are concerns because of their general toxicity and the fact they can enter the food chain as well as affecting the quality of water, soil and atmosphere. In the absence of well thought remediation plans, risk management strategies can give information needed to allow

\begin{tabular}{|l|}
\hline Deleted: these \\
\hline Deleted: and \\
\hline Deleted: re \\
\hline Deleted: C \\
\hline Deleted: are raised due to \\
\hline Deleted: human and animal \\
\hline Deleted: $\mathrm{P}$ \\
\hline Deleted: or or \\
\hline
\end{tabular}


stakeholders to decide options concerning disposal of these potentially harmful chemicals.

There have been numerous investigations involving the modelling of clay surfaces and adsorption of organic species [18-21l However, our interest lies in the understanding of how these molecules might bind to mineral surfaces and in establishing a high-throughput methodology that can efficiently investigate the many possible combinations of $\underline{\text { molecules with substrates. }}$

As a starting point, we choose the polychloro-dibenzo-pdioxins (PCDDs) family. The 76 members of this family (namely, congeners) have a different number of chlorine atoms replacing hydrogen atoms at different positions.

We then choose a clay substrate, of relevence to soils The di-octahedral 2:1 sheet silicate pyrophillite, $\mathrm{Al}_{4} \mathrm{Si}_{8} \mathrm{O}_{20}(\mathrm{OH})_{4}$. is a simple example of the family of clay minerals that form a significant fraction of many soil and rock types. Its [001] face was first considered.

Preliminary DFT calculations showed that the organic molecules do not chemisorb on the pyrophillite surface. Of course, DFT is not adapted to the accurate study of physisorption phenomena due to its poor treatment of dispersion. Therefore our potential based method was deemed ideal for such a study.

We now describe the choices of models, tools and resources, and then follow with a brief discussion of the automatisation procedures and comments on the scientific results.

\section{Model}

A classical model requires an electrostatic description of atoms as well as a parameterisation of the intra-molecular and the inter-molecular interactions.
Deleted: Our interest lies in understanding how these molecules bind to mineral surfaces and to establish a high-throughput methodology to efficiently

investigate many

molecules/substrates combinations.

Deleted: ther

Deleted: [38 - 42] 
Well established models for Zeolite and clay minerals make modeling the substrate a comparatively straightforward task. We chose the parameterisation of Lewis and Catlow [22] in which the electrostatics is described by the shell model of Dick and Overhauser

[9] The total charge is separated between the shell and the core. In the molecular dynamics simulations the shells were given a mass of $0.5 \mathrm{au}$.

For the molecules, we chose the CVFF model $[23]$ for the bonding interactions. The Deleted: [12] attribution of the electrostatic components was more complex. We used ab initio calculations (Gaussian code [24l) with the Dunning/Huzinaga valence double-zeta basis Deleted: g set on selected PCDDs'. From these we extracted the charge distribution to extrapolate point charges for all the PCDD. This simplified charge model is described in table 1.

\begin{tabular}{lc}
\hline \hline \multicolumn{1}{c}{ Specie } & Charge $(\mathrm{e})$ \\
\hline $\mathrm{H}$ & .20 \\
$\mathrm{Cl}$ & -.03 \\
$\mathrm{O}$ & -.50 \\
$\mathrm{C}(\mathrm{H}$ bonded $)$ & -.20 \\
$\mathrm{C}(\mathrm{Cl}$ bonded $)$ & .03 \\
$\mathrm{C}(\mathrm{O}$ bonded $)$ & .25 \\
\hline \hline
\end{tabular}

Table 1: Charge model (in e)

We generated the interaction parameters between the substrate and the adsorbed molecules by using the standard combination rules, which is a valid approach to obtain dispersion-repulsion interaction coefficients in the absence of strong bonding.

Our confidence in this model is confirmed by the excellent agreement of test calculations on the adsorption of simpler molecules (linear and cyclic aliphatic hydrocarbons) with 
experimental measurements $[18,25]$; in the case of cyclohexane, $-57 \mathrm{~kJ} \cdot \mathrm{mol}^{-1}$ compared to $-54 \mathrm{~kJ} \cdot \mathrm{mol}^{-1}$ at_ $423 \mathrm{~K}$.

\section{DL_POLY as an optimiser}

DL_POLY is the UK's national flagship package for molecular dynamics (MD) | simulations [26l, While it is especially good for simulating very large systems, its modular structure makes it very adaptable. It supports some form of energy optimisation (albeit not as efficiently as specialist codes such as GULP [27] or METADISE [6]). It is also $\mathrm{cml}$ enabled which allows for certain direct inter-operability with some DFT codes.

The choice of a MD code for a problem essentially based on optimisation might appear to be odd at first. However, our main objective is to tackle adsorption in realistic conditions, i.e. at various temperatures and with the involvement of water. Molecular Dynamics is $\underline{\text { the tool of choice for these more advanced problems. So, to maintain upward }}$ compatibility, we decided to use the same code for the current study as we would for the more advanced one, even though there is added complexity using a MD code to perform optimisation

Additionally, the choice was justified because the force field involved was designed with the aspects of the larger study in mind and is therefore relatively complex and not adaptable to GULP or METADISE codes without heavy recoding

\section{UCL Condor Pool}

This project is combinatorial in nature. That is, not only are there seventy-six non equivalent PCDD molecules, but the determination of reliable adsorption sites requires multiple starting configurations. Furthermore, the search space is large and the potential

Deleted: [16]

Deleted: [17]

Deleted: later

Deleted: This

Deleted: we d

Deleted: is

Deleted: and especially because that

Deleted: our main objective is to tackle adsorption in realistic

conditions, i.e. at various

temperatures and with of water. Molecular Dynamics is the tool of choice for these more advanced problems. So, to maintain upward compatibility, we decided to use the same code for the current study as we would for the more advanced one, at the cost of the early added complexity of using a MD code to perform optimisation

Deleted: N 
energy surface can be complex and thus contains many local minima. However, the systems are not overly large and each energy calculation is not too computationally demanding. A brute force approach to optimisation is therefore deemed appropriate and

Deleted: $\mathrm{t}$ Deleted: herefore, a indeed, affordable.

Deleted: blunt, heavy handed, or

Instead of using a supercomputer, we took advantage of the UCL Condor pool. This type of resource is ideal for this study, as it contains a very large amount of processors (at the time of writing 960 CPUs, operating under Windows $\mathrm{OS}_{\boldsymbol{z}}$

Another feature of the Condor setup is the DAGMAN workflow manager which allows complex workflows. In our case, it essentially made the analysis task automatic.

\section{Methodology}

First of all, three sets of independent calculations are required: molecule only (M), substrate only (S), molecule adsorbed on substrate $(\mathrm{M}+\mathrm{S})$. The adsorption energy is defined as a difference between the energy of these systems $\left(\mathrm{E}_{\mathrm{MS}^{-}}^{-} \mathrm{EM}^{-}-\mathrm{E}_{\mathrm{S}}\right)$

The two custom files node_list and atom_list are at the centre of the data structure and operate on the two modified DL_POLY input files FIELD and CONFIG. node_list contains seventy six lines, one for each PCCD, starting with a user provided molecule code, and then twenty two intermediate atom codes, in the order in which the atoms appear in the CONFIG file (these codes can be $\mathrm{H}, \mathrm{Cl}, \mathrm{O}, \mathrm{C}, \mathrm{CH}, \mathrm{CCl}$ or $\mathrm{CO}$ ). atom_list creates a link between these intermediary codes and the names used in the CONFIG $(H$, $\mathrm{Cl}, \mathrm{O}, \mathrm{C}$ ), as well as with the charges appearing in the FIELD file (as the carbon atoms can be allocated different charges). It also contains information concerning the bonding parameters, which need to be allocated in the FIELD file. 
1

2

3

4

5

6

7

8

9

10

A list of dedicated scripts reads the node_list and operates on the 76 corresponding directories.

The simpler scripts are just expansions of unix commands (list_cp, list_mv, list_grep). list_setdir generates the data structure (directories NODE_n-moleculecode, where $\mathrm{n}$ is an arbitrary molecule index, corresponding to the order in node_list). list_config replaces <list> flags in a modified CONFIG by the correct atom name and put the correct CONFIG in its directory. list_field replaces <list > and < harm> flags in the field by their value from the atom_list (atom name and charge, two specie bonds parameters). list_fire submit the jobs to the condor scheduler (via run.dlp files). list_rota, is an expanded version of list_config which additionally applies a rotation and/or translation to the flagged atoms of CONFIG (in our case of course those of the PCDD molecule).

These scripts are sufficient to optimise the structures of the isolated molecules.

As already mentioned, the case of the adsorbed molecules is slightly more complex as several starting configuration are needed. Each of these is carried sequentially, for each molecule. The script config_rota, a same directory, command line version of list_rota is used in conjunction with DAGMAN. list_fire is also updated to list_fire_dag. Practically, three sites of high symmetry were explored (above $\mathrm{Si}$, above $\mathrm{O}$, above "hexagon" centre, see figure 16) with 8 rotational configuration (by increment of pi/2), with 24 calculations per molecule.

\section{Results and discussion}

In Figure 15 we plot the adsorption energy of the 76 PCDDs. The molecule index is only meaningful in so far as it increases with the number of chlorine atoms. The horizontal lines are a guide for the eye and correspond to molecules with the same number of 
chlorine. It can be seen that the adsorption energy is strongly correlated to the number of chlorine. Each additional chlorine attached to the carbon rings leads on average to an

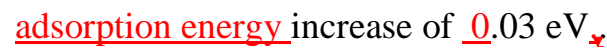

Furthermore, and as might have been expected, all of the molecules lie flat on the surface, see Figure 16.

We also observe that corrugation energy jncreases with the number of chlorine. The potential energy surface is very flat for the PCDD with a low $\mathrm{Cl}$ count (indeed the translational corrugation energy is $0 \pm 001 \mathrm{eV}$ for $\left.\mathrm{C}_{12} \mathrm{O}_{2} \mathrm{H}_{8}\right)$. At higher $\mathrm{Cl}$ count, the corrugation increases, up to $\underline{0} .07 \mathrm{eV}$ for $\mathrm{C}_{12} \mathrm{O}_{2} \mathrm{Cl}_{8}$. Therefore, the concept of the adsorption site is rather meaningless for molecules with numbers of $\mathrm{Cl}$ lower than 4 . For molecules with greater than $3 \mathrm{Cl}$ atoms, the PCDD almost systematically favours a position such as illustrated in Figure 16, with the centre of mass of the molecule situated above a surface oxygen, and where the benzene rings sit inside the quasi-hexagon of the surface top layer.

As well as the modelling of molecules that are in contact with surfaces, we are also concerned with the interaction of nanoparticles.

Deleted: in adsorption energy

Deleted: could

Deleted: s

Deleted: ay

Deleted: the

Deleted:

Deleted: do

Deleted: a

Deleted:

Deleted: of

Deleted: .

Deleted: N

Deleted: N

Deleted: higher than 3
Deleted: In addition

Deleted: ,

Deleted: to

\section{Molecular dynamics simulations of the structural changes that occur during aggregation of mineral oxide and carbonate nanoparticles}

DL_POLY has been used by other groups to study the structure and stability on minerals on the nano-scale. Spano et al found computational evidence of $\mathrm{ZnS}$ forming bubble type 
clusters $\left[28 \mathrm{Z}(\mathrm{ZnS})_{\mathrm{n}}\right.$ clusters, with $\mathrm{n}$ ranging from 10 to 47, were energy minimised using a simulated annealing technique. Spano et al found that spheroidal (bubble) clusters with only three coordinated atoms are more stable than the bulk structure. If the number of $\mathrm{ZnS}$ units is increased to 60 then the appearance of onion structures occur [29l, This is a $(\mathrm{ZnS})_{12}$ inside a bubble of $(\mathrm{ZnS})_{48}$. Banfield and co workers have also considered $\mathrm{ZnS}$ nanoparticles, typically $\mathrm{n}=360-400$, making the diameter of the $\mathrm{ZnS}$ crystal around 3nm, which is comparable to experimental synthesised particles [30] The Banfield group started using molecular dynamics to study the phase stability of $\mathrm{ZnS}$ nanoparticles in vacuum and in the presence of water. They found that the wurtzite $(\mathrm{ZnS})$ nanoparticles smaller than $7 \mathrm{~nm}$ are more stable than sphalerite nanoparticles in vacuum at $300 \mathrm{~K}$. However adsorption of water onto the $\mathrm{ZnS}$ nanoparticle surface stabilizes the sphalerite structure. Zhang et al 311 predicted anhydrous $\mathrm{ZnS}$ nanoparticles to have a highly disordered structure. Again these nanoparticles had a diameter of around $3 \mathrm{~nm}$. The adsorption of water, however, onto the nanoparticle surface results in the formation of a relatively crystalline core. Zhang and Banfield showed that this phase transformation of sphalerite to wurtzite could be caused by nanoparticle aggregation and coarsening $[32$ These $3 \mathrm{~nm} \mathrm{ZnS}$ nanoparticles were randomly orientated with respect to each other and allowed to aggregate to form a larger nanoparticle. Following aggregation the coarsened particle adopts a near-amorphous structure that transforms rapidly to wurtzite. This work by Banfield and co-workers has provided a link between atomistic simulation and the forces that cause their aggregation, i.e. colloidal chemistry. We have used DL_POLY to extend the range of materials to simulate the aggregation process of two different materials, $\mathrm{MgO}$ and calcite $\left(\mathrm{CaCO}_{3}\right)$ nanoparticles. First we will describe the potentials
Deleted: [18]

Deleted: [19]

Deleted: [21]

Deleted: [22] 
used to describe the interatomic interactions and then describe how we set up our systems for aggregation.

The potential model used for simulating the magnesium-oxygen interaction is that of Lewis and Catlow [33] and has been used before to simulate MgO surfaces [34, 35] and MgO nanocrystals [36] The potential model used for simulating the calcium-carbonate interactions in calcite is that of Pavese et al 377 and has been used in the past to simulate calcite surfaces [38] and also calcite nanoparticles [39], All simulations in this section employ the shell model of Dick and Overhauser [9]. Where the shells are in the oxygen

Deleted: [23] Deleted: [24, 25] Deleted: [26]

Deleted: [27]

Deleted: [28]

Deleted: [29]

Deleted: [11] species only and have a mass of 0.2 au in $\mathrm{CaCO}_{3}$ and 0.5 in $\mathrm{MgO}$, this represents the electronic charge cloud connected by a harmonic spring to a core containing most of the anion's mass.

We have begun studying the structure and aggregation using molecular dynamics simulations by modelling an eight nanoparticle system. Each of the nanoparticles in the simulation is the same distance apart. The simulation was performed in vacuum with no periodic boundary conditions. The molecular dynamic simulation was performed in a canonical ensemble at $300 \mathrm{~K}$ and ambient pressure using a time step of $0.1 \mathrm{fs}$. Each simulation was run for $1 \mathrm{~ns}$ with a $2 \mathrm{ps}$ equilibration period.

\section{Aggregation of Magnesium Oxide nanoparticles}

Each $\mathrm{MgO}$ nanoparticle had a $0.6 \mathrm{~nm}$ in diameter and the simulation started with them being $2.3 \mathrm{~nm}$ apart. The starting configuration is shown in figure 17. We have 8 cubes of $\mathrm{MgO}$ as the most stable surface grows along the $\{100\}$ plane [6]. We can plot the potential energy of our simulation as it evolves over time (figure 18). The starting 
configuration has a potential energy of $-482.1 \mathrm{~kJ} / \mathrm{mol}$ of $\mathrm{Mg}$ ions and then drops rapidly in-between $30-55 \mathrm{ps}$ to $-484.4 \mathrm{~kJ} / \mathrm{mol}$. This decrease in potential energy corresponds to the initial aggregation of $\mathrm{MgO}$ nanoparticles. Figure 19 shows a snapshot of the MD simulation at 30ps. The particles retain their bulk-like structure and well ordered surfaces. At 30ps single $\mathrm{MgO}$ nanoparticles are starting to aggregate together in pairs. The aggregation process seems very ordered and is dominated by orientation along the $\{100\}$ plane. This snapshot is also interesting because it also shows that the merging nanoparticles will join at the corners first. The pair of nanoparticles that are circled in figure 19 are a pair that drifts further and further away from the rest of the nanoparticles and don't aggregate with the other six nanoparticles. Since they are so far away in some of the snapshots it is not practical to show them in some of the pictures. At 60ps figure 20 shows a stabilisation of potential energy at around $-484.4 \mathrm{~kJ} / \mathrm{mol}$. Figure 20 shows a snapshot of the MD simulation at 60ps and shows the aggregation of a single $\mathrm{MgO}$ nanoparticle to a pair. The snapshot again illustrates the general result that aggregation has initially occurred along the corners of the nanoparticles. The nanoparticles find their preferred face centre cubic site at 220ps (figure 21) when the potential decreases to around $-484.6 \mathrm{~kJ} / \mathrm{mol}$. The increased ordering of aggregation along the $\{100\}$ plan continues when the potential energy decreases to $-485.8 \mathrm{~kJ} / \mathrm{mol}$ when a pair of nanoparticles aggregate to the triplet at 275ps (figure 22). The final configuration occurs at 460ps (figure 23). Here the sixth nanoparticle joins the cluster of five. The two nanoparticles circled in Figure 19 were in fact too far away to become attracted to the rest of them. This aggregation process has also shown that when crystal growth occurs in $\mathrm{MgO}$ there are the chances of kinks and steps being formed. 
We can compare any structural changes that may have occurred during aggregation by comparing the radial distribution functions (RDF) of aggregated nanoparticles with bulk $\mathrm{MgO}$. Figure 24 shows the RDF of the (a) aggregated MgO nanoparticle and (b) bulk $\mathrm{MgO}$. The RDF's of the aggregated $\mathrm{MgO}$ nanoparticle and bulk $\mathrm{MgO}$ are very similar with the first three peaks showing $\mathrm{Mg}-\mathrm{O}$ bond distances at $0.21,0.35$ and $0.45 \mathrm{~nm}$ in both

graphs. These interatomic distances agree with x-ray diffraction experiments $[40,41]$ Deleted: $[30,31]$

\section{Aggregation of calcite nanoparticles}

Eight calcite nanoparticles that are $1.6 \mathrm{~nm}$ in diameter are placed $1.7 \mathrm{~nm}$ apart. The starting configuration is shown in figure 25 . The simulation was run for 1 ns. Figure 28 shows the potential energy of the system as a function of MD time. The simulation starts with a potential energy of $-715.3 \mathrm{~kJ} / \mathrm{mol}$ of calcium ions. As the simulation evolves the first drop in potential energy occurs at around 50ps. This corresponds to figure 27 which shows that before aggregation occurs the individual nanoparticles will relax on their own to a potential energy of $-715.6 \mathrm{~kJ} / \mathrm{mol}$, which corresponds to a more disordered structure. Once the calcite particles are relaxed aggregation occurs in pairs. At around $67 \mathrm{ps}$ the eight nanoparticles have formed four nanoparticles and the energy lowers to $716.9 \mathrm{~kJ} / \mathrm{mol}$. Figure 28 shows a snapshot of aggregation at $87 \mathrm{ps}$. The potential energy of 
this system continues to lower, $-718.2 \mathrm{~kJ} / \mathrm{mol}$, as the four calcite nanoparticles start to form two larger nanocrystals. The two larger calcite nanoparticles now become attracted to each other and again as they merge the potential energy drops to $-719.9 \mathrm{~kJ} / \mathrm{mol}$ at around 140ps (figure 29. The final configuration occurs at 180ps and the potential energy stabilises at $-721.0 \mathrm{~kJ} / \mathrm{mol}$. Figure 30 shows the aggregated nanoparticle, which has lost its symmetry. The final nanoparticle has a longest diameter of $4.17 \mathrm{~nm}$ and a width of $1.816 \mathrm{~nm}$. Figure 31a shows the RDF of the final aggregated calcite nanoparticle. When compared with the RDF of bulk calcite (Figure 31b) it has much less order and is more amorphous then the crystalline bulk. The peaks in the RDF bulk calcite shows the same Ca-O bond distances, $0.23,0.33,0.41 \mathrm{~nm}$, as found experimentally $[42,43]$ The average coordination number for a calcium ion in the aggregated nanoparticle is calculated to be around 6.5, which is slightly higher then the coordination number for bulk calcite which is 6. This suggests that the amorphous nanoparticle is more closely related to calcite, rather than aragonite which has a calcium ion coordination number of 8 .

The difference between an aggregated calcite and an aggregated $\mathrm{MgO}$ is that $\mathrm{MgO}$ keeps its bulk-like structure and still has crystalline characteristics. The RDF shows sharp peaks whereas the RDF of the aggregated calcite is more amorphous with only two peaks. The atoms of the calcite nanoparticle seems to be more disordered and has no structural preference for linking different particles whereas the aggregated $\mathrm{MgO}$ has a very uniform structure.

There has been a great deal of debate in the literature suggesting that biomineralisation occurs from amorphous calcium carbonate [44-47l. We have shown that very small calcium carbonate nanoparticles are more stable when amorphous and also when they 
aggregate together their morphology changes drastically. Therefore this aggregation process could promote the exotic morphologies seen in biomineralistion. In future studies we set out to quantify this aggregation process.

\section{Conclusions}

DL_POLY has great scope for our molecular dynamics simulation experiments. We have been able to calculate both the free energy of nanoparticle aggregation and the activation free energy of individual atom migration. DL_POLY has also been shown to be versatile when used as an energy optimiser and for investigating adsorption of molecules on surfaces.

Application of DL_POLY has allowed us to investigate oxygen atom migration at the stable $\{111\}$ surface of ceria. The oxygen migration is shown to be much more energetically favoured at the $\{111\}$ surface than the bulk when via subsurface oxygen vacancies, and the activation free energy of migration was found to be the same in all directions. When considering the effect of neighbouring charge compensating $\mathrm{Ce}^{3+}$ ions the oxygen was found to be less mobile and no longer isotopic. 
We have determined the adsorption energy and sites of the 76 PCDD congeners on a pyrophilite surface, using DL_POLY. The molecules are weakly bonded and the corrugation is low. The variation in energy with adsorption sites is small. The main factor controlling the adsorption energy is the number of chlorine atoms.

The simplicity of the PCDDs adsorption on this surface means that the process can be modelled very simply and parameterised for use in large scale soil remediation models.

We have investigated the differences between calcite and $\mathrm{MgO}$ nanoparticle aggregation. The difference between an aggregated calcite and an aggregated $\mathrm{MgO}$ is that $\mathrm{MgO}$ keeps its bulk-like structure and aggregates in specific orientations. In contrast,_aggregated calcite is more amorphous and does not have any noticeable preferred directionality. The structure of the calcite nanoparticle seems to be more mobile whereas the aggregated $\mathrm{MgO}$ has a very uniform structure.

In summary, DL_POLY allows us to carry-out sophisticated molecular dynamics simulations, intricate computational calculations and gives us scope for further investigatory work. Importantly, these applications of DL_POLY are providing a useful complement to experiments.
Deleted: not

Deleted: real

\section{Deleted: is}


1

2

3

4

5

6

7

8

9

10

11

12

13

14

15

16

17

18

19

20

21

22

23

24

25

26

27

28

29

30

31

32

33

34

35

36

37

38

39

40

41

42

43

44

45

46

47

48

49

50

51

52

53

54

55

56

57

58

59

60

http://mc.manuscriptcentral.com/tandf/jenmol 
References:

1. White, T.O., R.P. Bruin, J. Wakelin, C. Chapman, D. Osborn, P. Murray-Rust, E. Artacho, M.T. Dove, and M. Calleja. eScience methods for the combinatorial chemistry problem of adsorption of pollutant organic molecules on mineral surfaces. Proceedings of the UK e-Science All Hands Meeting 2005. (2005)

2. Deluga, G.A., J.R. Salge, L.D. Schmidt, and X.E. Verykios. Renewable hydrogen from ethanol by autothermal reforming. Science. 303. 5660. (2004)

3. Otsuka, K., T. Ushiyama, and I. Yamanaka. Partial Oxidation of Methane Using the Redox of Cerium Oxide. Chemistry Letters. 9. (1993)

4. Trovarelli, Catalysis by Ceria and Related Materials. 2002, London: Imperial College Press.

5. Park, S.D., J.M. Vohs, and R.J. Gorte. Direct oxidation of hydrocarbons in a solid-oxide fuel cell. Nature. 404.6775. (2000)

6. Watson, G.W., E.T. Kelsey, N.H. deLeeuw, D.J. Harris, and S.C. Parker. Atomistic simulation of dislocations, surfaces and interfaces in $\mathrm{MgO}$. Journal of the Chemical Society-Faraday Transactions. 92. 3. (1996)

7. Balducci, G., J. Kaspar, P. Fornasiero, M. Graziani, M.S. Islam, and J.D. Gale. Computer simulation studies of bulk reduction and oxygen migration in $\mathrm{CeO} 2-$ ZrO2 solid solutions. Journal of Physical Chemistry B. 101. 10. (1997)

8. Sayle, T.X.T., S.C. Parker, and C.R.A. Catlow. Surface Segregation of Metal-Ions in Cerium Dioxide. Journal of Physical Chemistry. 98. 51. (1994)

9. Dick, A.W. and B.G. Overhauser. Theory of dielectric constants of alkali halide crystals. Physical Reviews. 112. 1. (1958)

10. Kerisit, S. and S.C. Parker. Free energy of adsorption of water and metal ions on the $\{1014\}$ calcite surface. Journal of the American Chemical Society. 126. 32. $\underline{(2004)}$

11. Balducci, G., M.S. Islam, J. Kaspar, P. Fornasiero, and M. Graziani. Bulk reduction and oxygen migration in the ceria-based oxides. Chemistry of Materials. 12. 3. (2000)

12. Harris, D.J., M.Y. Lavrentiev, J.H. Harding, N.L. Allan, and J.A. Purton. Novel exchange mechanisms in the surface diffusion of oxides. Journal of PhysicsCondensed Matter. 16. 13. (2004)

13. Nolan, M., S. Grigoleit, D.C. Sayle, S.C. Parker, and G.W. Watson. Density functional theory studies of the structure and electronic structure of pure and defective low index surfaces of ceria. Surface Science. 576. 1-3. (2005)

14. Nolan, M., S.C. Parker, and G.W. Watson. The electronic structure of oxygen vacancy defects at the low index surfaces of ceria. Surface Science. 595. 1-3. $\underline{(2005)}$

15. Nolan, M., S.C. Parker, and G.W. Watson. Reduction of NO2 on ceria surfaces. Journal of Physical Chemistry B. 110. 5. (2006)

16. Nolan, M., S.C. Parker, and G.W. Watson. CeO2 catalysed conversion of $\mathrm{CO}$, $\mathrm{NO} 2$ and NO from first principles energetics. Physical Chemistry Chemical Physics. 8.2.(2006)

Formatted: Font: Italic

Formatted: Font: Italic

Formatted: Font: Bold

Formatted: Font: Italic

Formatted: Font: Italic

Formatted: Font: Italic

Formatted: Font: Bold

Formatted: Font: Italic

Formatted: Font: Bold

Formatted: Font: Italic

Formatted: Font: Bold

Formatted: Font: Italic

Formatted: Font: Bold

Formatted: Font: Italic

Formatted: Font: Bold

Formatted: Font: Italic

Formatted: Font: Bold

Formatted: Font: Italic

Formatted: Font: Bold

Formatted: Font: Italic

Formatted: Font: Bold

Formatted: Font: Italic

Formatted: Font: Bold

Formatted: Font: Italic

Formatted: Font: Bold

Formatted: Font: Italic

Formatted: Font: Bold

Formatted: Font: Italic

Formatted: Font: Bold 
17. Parker, S.C., S. Kerisit, A. Marmier, S. Grigoleit, and G.W. Watson. Modelling inorganic solids and their interfaces: A combined approach of atomistic and electronic structure simulation techniques. Faraday Discussions. 124. (2003)

18. Keldsen, G.L., J.B. Nicholas, K.A. Carrado, and R.E. Winans. Molecular Modeling of the Enthalpies of Adsorption of Hydrocarbons on Smectite Clay. Journal of Physical Chemistry. 98. 1. (1994)

19. Skipper, N.T., P.A. Lock, J.O. Titiloye, J. Swenson, Z.A. Mirza, W.S. Howells and F. Fernandez-Alonso. The structure and dynamics of 2-dimensional fluids in swelling clays. Chemical Geology. 230. 3-4. (2006)

20. Yu, C.H., S.Q. Newton, M.A. Norman, L. Schafer, and D.M. Miller. Molecular dynamics simulations of adsorption of organic compounds at the clay mineral/aqueous solution interface. Structural Chemistry. 14. 2. (2003)

21. Zeng, Q.H., A.B. Yu, G.Q. Lu, and R.K. Standish. Molecular dynamics simulation of organic-inorganic nanocomposites: Layering behavior and interlayer structure of organoclays. Chemistry of Materials. 15. 25. (2003)

22. Lewis, G.V. and C.R.A. Catlow. Potential Models for Ionic Oxides. Journal of Physics C-Solid State Physics. 18. 6. (1985)

23. Dauberosguthorpe, P., V.A. Roberts, D.J. Osguthorpe, J. Wolff, M. Genest, and A.T. Hagler. Structure and Energetics of Ligand-Binding to Proteins Escherichia-Coli Dihydrofolate Reductase Trimethoprim, a Drug-Receptor System. Proteins-Structure Function and Genetics. 4. 1. (1988)

24. Frisch, M.J., G.W. Trucks, H.B. Schlegel, G.E. Scuseria, M.A. Robb, J.R. Cheeseman, J. Montgomery, J. A., T. Vreven, K.N. Kudin, J.C. Burant, J.M. Millam, S.S. Iyengar, J. Tomasi, V. Barone, B. Mennucci, M. Cossi, G. Scalmani, N. Rega, G.A. Petersson, H. Nakatsuji, M. Hada, M. Ehara, K. Toyota, R. Fukuda, J. Hasegawa, M. Ishida, T. Nakajima, Y. Honda, O. Kitao, H. Nakai, M. Klene, X. Li, J.E. Knox, H.P. Hratchian, J.B. Cross, V. Bakken, C. Adamo, J. Jaramillo, R. Gomperts, R.E. Stratmann, O. Yazyev, A.J. Austin, R. Cammi, C. Pomelli, J.W. Ochterski, P.Y. Ayala, K. Morokuma, G.A. Voth, P. Salvador, J.J. Dannenberg, V.G. Zakrzewski, S. Dapprich, A.D. Daniels, M.C. Strain, O. Farkas, D.K. Malick, A.D. Rabuck, K. Raghavachari, J.B. Foresman, J.V. Ortiz, Q. Cui, A.G. Baboul, S. Clifford, J. Cioslowski, B.B. Stefanov, G. Liu, A. Liashenko, P. Piskorz, I. Komaromi, R.L. Martin, D.J. Fox, T. Keith, M.A. AlLaham, C.Y. Peng, A. Nanayakkara, M. Challacombe, P.M.W. Gill, B. Johnson, W. Chen, M.W. Wong, C. Gonzalez, and J.A. Pople, Gaussian 03, Revision C.02. 2004, Gaussian, Inc.: Wallingford CT.

25. Zhukova, A.I., S.V. Bondarenko, and Y.I. Tarasevich. Study of Selectivity of Natural Sorbents with Respect to Hydrocarbons. Ukrainskii Khimicheskii Zhurnal.42.7.(1976)

26. Smith, W. and T.R. Forester. DL POLY 2.0: A general-purpose parallel molecular dynamics simulation package. Journal of Molecular Graphics. 14. 3. $\underline{(1996)}$

27. Gale, J.D. and A.L. Rohl. The General Utility Lattice Program (GULP). Molecular Simulation. 29. 5. (2003)

28. Spano, E., S. Hamad, and C.R.A. Catlow. Computational evidence of bubble $\mathrm{ZnS}$ clusters. Journal of Physical Chemistry B. 107. 38. (2003)

Formatted: Font: Italic Formatted: Font: Bold

Formatted: Font: Italic

Formatted: Font: Bold

Formatted: Font: Italic

Formatted: Font: Bold

Formatted: Font: Italic

Formatted: Font: Bold

Formatted: Font: Italic

Formatted: Font: Bold

Formatted: Font: Italic

Formatted: Font: Bold

Formatted: Font: Italic

Formatted: Font: Bold
Formatted: Font: Italic

Formatted: Font: Italic

Formatted: Font: Bold

Formatted: Font: Italic

Formatted: Font: Bold

Formatted: Font: Italic

Formatted: Font: Bold

Formatted: Font: Italic

Formatted: Font: Bold 
29. Spano, E., S. Hamad, and C.R.A. Catlow. ZnS bubble clusters with onion-like structures. Chemical Communications. 7. (2004)

30. Zhang, H.Z., F. Huang, B. Gilbert, and J.F. Banfield. Molecular dynamics simulations, thermodynamic analysis, and experimental study of phase stability of zinc sulfide nanoparticles. Journal of Physical Chemistry B. 107. 47. (2003)

31. Zhang, H.Z., B. Gilbert, F. Huang, and J.F. Banfield. Water-driven structure transformation in nanoparticles at room temperature. Nature. 424. 6952. (2003)

32. Zhang, H.Z. and J.F. Banfield. Aggregation, coarsening, and phase transformation in $\mathrm{ZnS}$ nanoparticles studied by molecular dynamics simulations. Nano Letters. 4. 4. (2004)

33. Lewis, G.V. and C.R.A. Catlow. Potential model for ionic oxides. Journal of Physical Chemistry: Solid State Physics. 18. (1985)

34. de Leeuw, N.H. and S.C. Parker. Molecular-dynamics simulation of MgO surfaces in liquid water using a shell-model potential for water. Physical Review B. 58. 20. (1998)

35. Harris, D.J., J.H. Harding, and S.C. Parker. Simulations of surfaces and interfaces in MgO. Radiation Effects and Defects in Solids. 151. 1-4. (1999)

36. Sayle, D.C., J.A. Doig, S.A. Maicaneanu, and G.W. Watson. Atomistic structure of oxide nanoparticles supported on an oxide substrate. Physical Review B. 65. 24. $\underline{(2002)}$

37. Pavese, A., M. Catti, S.C. Parker, and A. Wall. Modelling of the thermal dependence of structural and elastic properties of calcite, $\mathrm{CaCO}$. Physics and Chemistry of Minerals. 23. 2. (1996)

38. Kerisit, S. and S.C. Parker. Free energy of adsorption of water and calcium on the $\{10(1)$ over-bar-4\} calcite surface. Chemical Communications. 1. (2004)

39. Kerisit, S., D.J. Cooke, D. Spagnoli, and S.C. Parker. Molecular dynamics simulations of the interactions between water and inorganic solids. Journal of Materials Chemistry. 15. 14. (2005)

40. Bragg, W.L. Crystal structure. Nature. 105. (1920)

41. Tsirel'son, V.G.A., A.S.;Abramov, Yu.A.;Belokoneva, E.L.;Kitaneh, R.;Feil, D. X-ray and electron diffraction study of Mg O. Acta Crystallographica B. 54. (1998)

42. Maslen, E.N.S.t., V.A.;Strel'tsova, N.R. X-ray study of the electron density in calcite, CaCO3. Acta Crystallographica B. 49. (1993)

43. Wyckoff, R.W.G. The crystal structures of some carbonates of the calcite group. American Journal of Science, Serie 50. (1920)

44. Addadi, L., S. Raz, and S. Weiner. Taking advantage of disorder: Amorphous calcium carbonate and its roles in biomineralization. Advanced Materials. 15. 12. (2003)

45. Faatz, M., F. Grohn, and G. Wegner. Amorphous calcium carbonate: Synthesis and potential intermediate in biomineralization. Advanced Materials. 16. 12. (2004)

46. Weiner, S., Y. Levi-Kalisman, S. Raz, and L. Addadi. Biologically formed amorphous calcium carbonate. Connective Tissue Research. 44. (2003)

47. Xu, X.R., J.T. Han, and K. Cho. Formation of amorphous calcium carbonate thin films and their role in biomineralization. Chemistry of Materials. 16. 9. (2004)

Formatted: Font: Italic

Formatted: Font: Italic

Formatted: Font: Bold

Formatted: Font: Italic

Formatted: Font: Bold

Formatted: Font: Italic

Formatted: Font: Bold

Formatted: Font: Italic

Formatted: Font: Bold

Formatted: Font: Italic

Formatted: Font: Bold

Formatted: Font: Italic

Formatted: Font: Bold

Formatted: Font: Italic

Formatted: Font: Bold

Formatted: Font: Italic

Formatted: Font: Bold

Formatted: Font: Italic

Formatted: Font: Italic

Formatted: Font: Bold

Formatted: Font: Italic

Formatted: Font: Bold

Formatted: Font: Italic

Formatted: Font: Bold

Formatted: Font: Italic

Formatted: Font: Bold

Formatted: Font: Italic

Formatted: Font: Bold

Formatted: Font: Italic

Formatted: Font: Bold

Formatted: Font: Italic

Formatted: Font: Bold

Formatted: Font: Italic

Formatted: Font: Bold

Formatted: Font: Italic

Formatted: Font: Bold 


\section{Table of Figures}

Figure 1: Oxygen Atom Migrating in The Middle of $\{111\}$ Surface Slab

Figure 2: Energy Profile of Bulk Oxygen Migration

Figure 3: Plot Showing Constrained Force Experienced by the Migrating Atom over Distance Travelled in Bulk Ceria.

Figure 4: Oxygen Atom Migrating in the $\{111\}$ Surface.

Figure 5: Energy Profile of Oxygen Migration in the $\{111\}$ Surface.

Figure 6: Energy Profile of Oxygen Migration from Bulk to Just Under the $\{111\}$

Surface.

Figure 7 (a) Schematic of oxygen hopping across the surface.

Figure 7 (b) Schematic of oxygen moving through the surface.

Figure 8: Pathway of Concerted Mechanism

Figure 9a: Before migration and with Surface Vacancy in place.

Figure 9b: After Oxygen atom, originally under the surface between $\mathrm{Ce}^{3+}$ ions, has migrated to the surface leaving vacancy just under the surface and between $\mathrm{Ce}^{3+}$ ions.

Figure 10: Energy Profile of Oxygen atom migration between $\mathrm{Ce}^{3+}$ ions onto the Surface.

Figure. 11a: Before migration and with Surface Vacancy in place.

Figure. 11b: After. Oxygen atom, originally under the surface and below the vacancy, has migrated to the surface leaving vacancy just under the surface and below the left $\mathrm{Ce}^{3+}$ ion.

Figure 12: Energy Profile of Oxygen atom migration From under the Left $\mathrm{Hand}_{\mathrm{Ce}}{ }^{3+}$ ion into the Surface Vacancy between Compensating $\mathrm{Ce}^{3+}$ ions.

Figure 13a: Before migration and with Surface Vacancy in place.

Figure 13b: After. Oxygen atom, originally under the surface between $\mathrm{Ce}^{3+}$ ions, has migrated to the surface leaving vacancy just under the surface and between $\mathrm{Ce}^{3+}$ ions.

Figure 14: Energy Profile of Oxygen atom migration From under the Left $\mathrm{Hand}^{\mathrm{Ce}^{3+}}$ ion into the Surface Vacancy between Compensating $\mathrm{Ce}^{3+}$ ions.

Figure 15: Adsorption energy of the 76 PCDD 
Figure 16: Adsorption position of a PCDD congener. The surface is parallel to the page. The shaded atoms represent the lower layers. Surface: Si Large light grey, O small dark grey. Molecule: $\mathrm{H}$ white, $\mathrm{Cl}$ light grey, $\mathrm{C}$ grey, $\mathrm{O}$ dark grey.

Figure 17: Initial configuration of eight $\mathrm{MgO}$ nanoparticles before aggregation.

Figure 18: Potential energy per magnesium ion against time of an MD simulation of 8 $\mathrm{MgO}$ nanoparticles

Figure 19: A snapshot of the MD simulation at 30ps for the $\mathrm{MgO}$ system

Figure 20: A snapshot of the MD simulation at 60ps for the $\mathrm{MgO}$ system

Figure 21: A snapshot of the MD simulation at 220ps for the $\mathrm{MgO}$ system

Figure 22: A snapshot of MD simulation at 275ps for the $\mathrm{MgO}$ system

Figure 23: Final configuration of the aggregated $\mathrm{MgO}$ nanoparticles

Figure 24: $\mathrm{RDF}$ of the (a) aggregated $\mathrm{MgO}$ nanoparticles and (b) bulk $\mathrm{MgO}$

Figure 25: Initial configuration of calcite nanoparticles for MD simulation

Figure 26: Potential energy per calcium ion against time of an MD simulation of 8 calcite nanoparticles

Figure 27: A snapshot of the MD simulation at 50ps for the calcite $1.6 \mathrm{~nm}$ system

Figure 28: A snapshot of the MD simulation at $87 \mathrm{ps}$ for the calcite $1.6 \mathrm{~nm}$ system

Figure 29: A snapshot of the MD simulation at 140ps for the calcite $1.6 \mathrm{~nm}$ system

Figure 30: Final configuration of the aggregated calcite nanoparticles

Figure 31: RDF of the (a) aggregated calcite nanoparticles and (b) bulk calcite 


\section{Page 81 of 158}

1

2

3
4

4

5

6
7

8

9

10

11

12
13

14

15

16

17

18

19

20

21

22

23

24

25

26

27

28

29

30

34

35

36

37

38

39

40

41

42

43

44

45

46

47

48

49

50

51

52

53

54

55

56

57

58

59

60

http://mc.manuscriptcentral.com/tandf/jenmol 


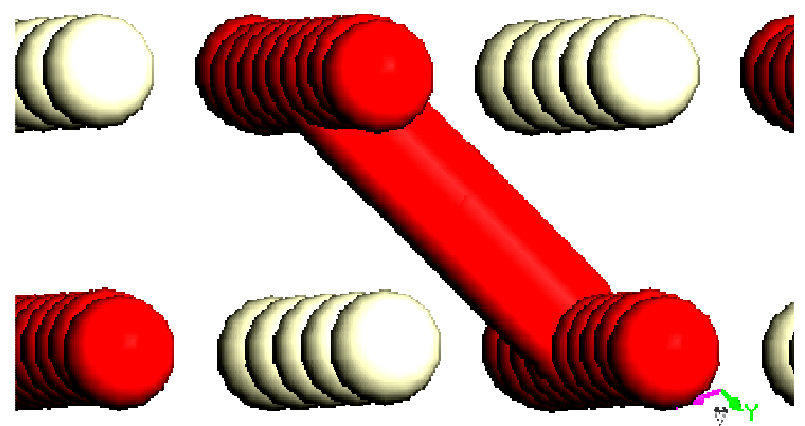

Figure 1: Oxygen Atom Migrating in The Middle of $\{111\}$ Surface Slab

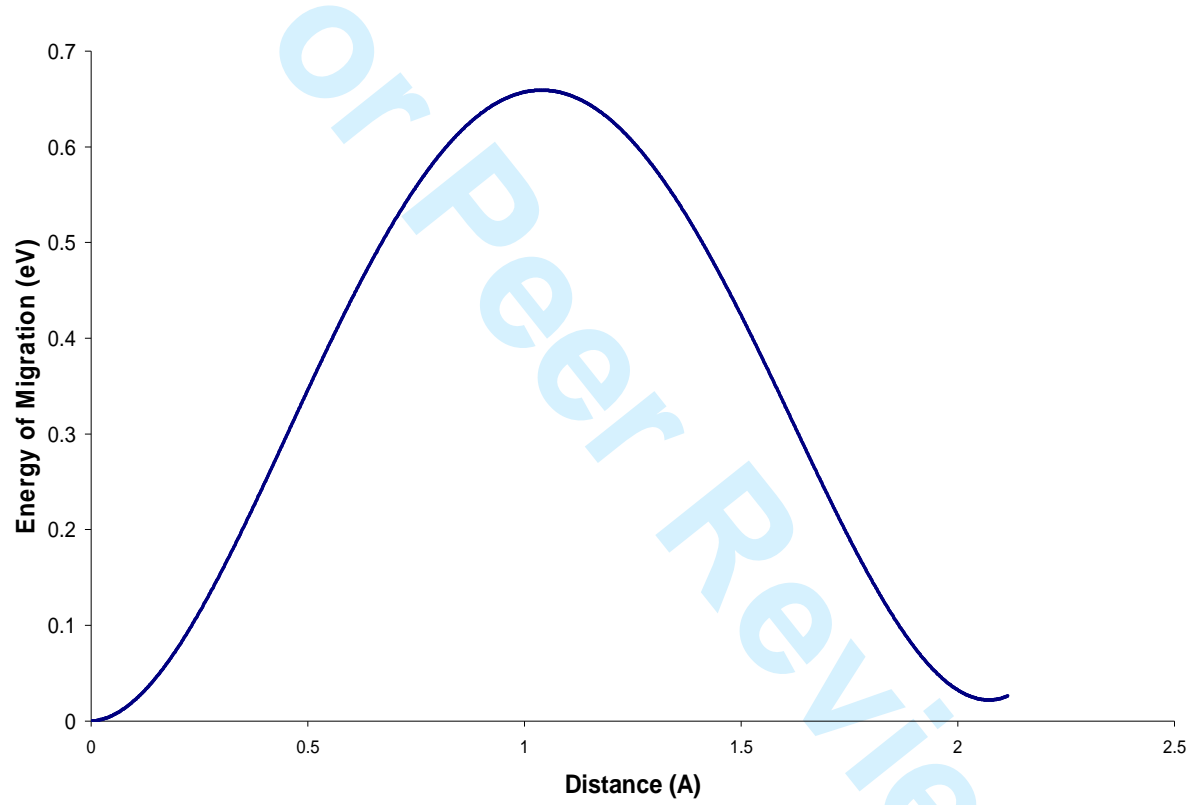

Figure 2: Energy Profile of Bulk Oxygen Migration 
1

2

3

4

5

6

7

8

9

10

11

12

13

14

15

16

17

18

19

20

21

22

23

24

25

26

27

28

29

30

31

32

33

34

35

36

37

38

39

40

41

42

43

44

45

46

47

48

49

50

51

52

53

54

55

56

57

58

59

60

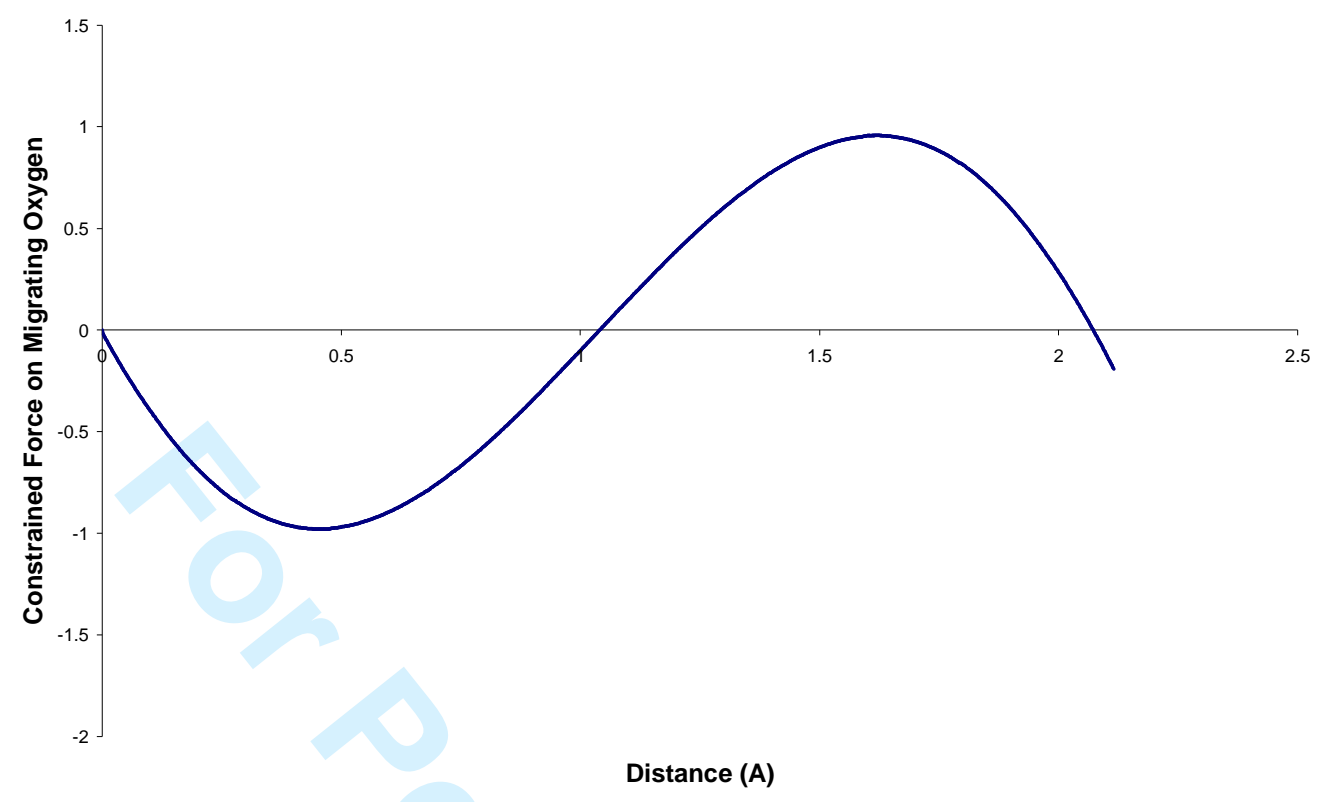

Figure 3: Plot Showing Constrained Force Experienced by the Migrating Atom (in eV / Å) over Distance Travelled in Bulk Ceria

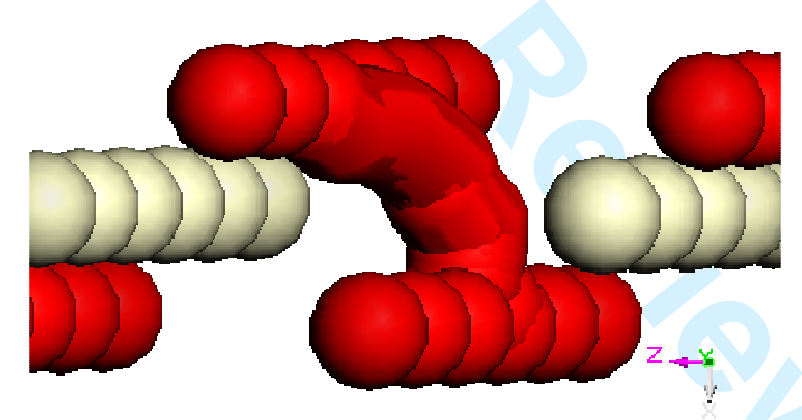

Figure 4: Oxygen Atom Migrating in the $\{111\}$ Surface 


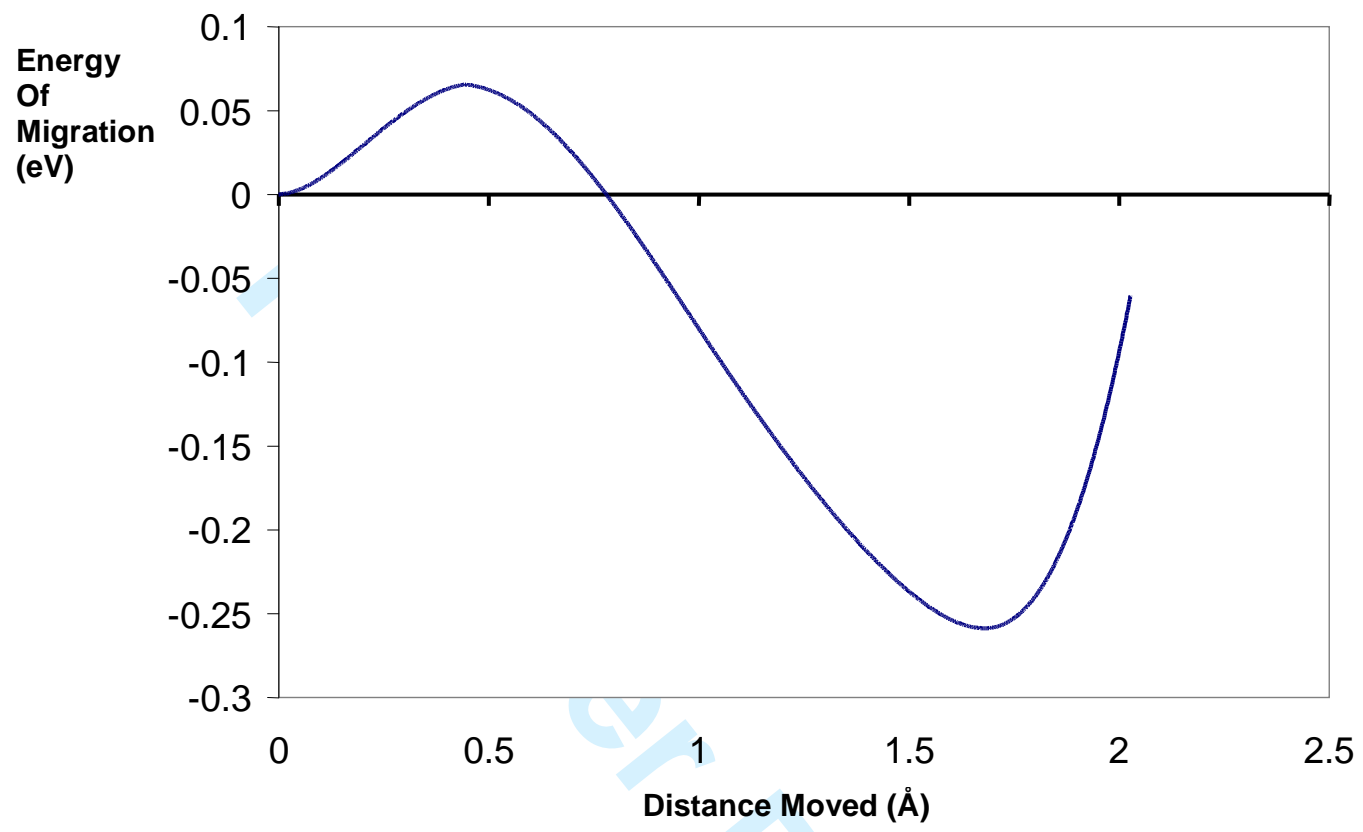

Figure 5: Energy Profile of Oxygen Migration in the $\{111\}$ Surface. 
1

2

3

4

5

6

7

8

9

10

11

12

13

14

15

16

17

18

19

20

21

22

23

24

25

26

27

28

29

30

31

32

33

34

35

36

37

38

39

40

41

42

43

44

45

46

47

48

49

50

51

52

53

54

55

56

57

58

59

60

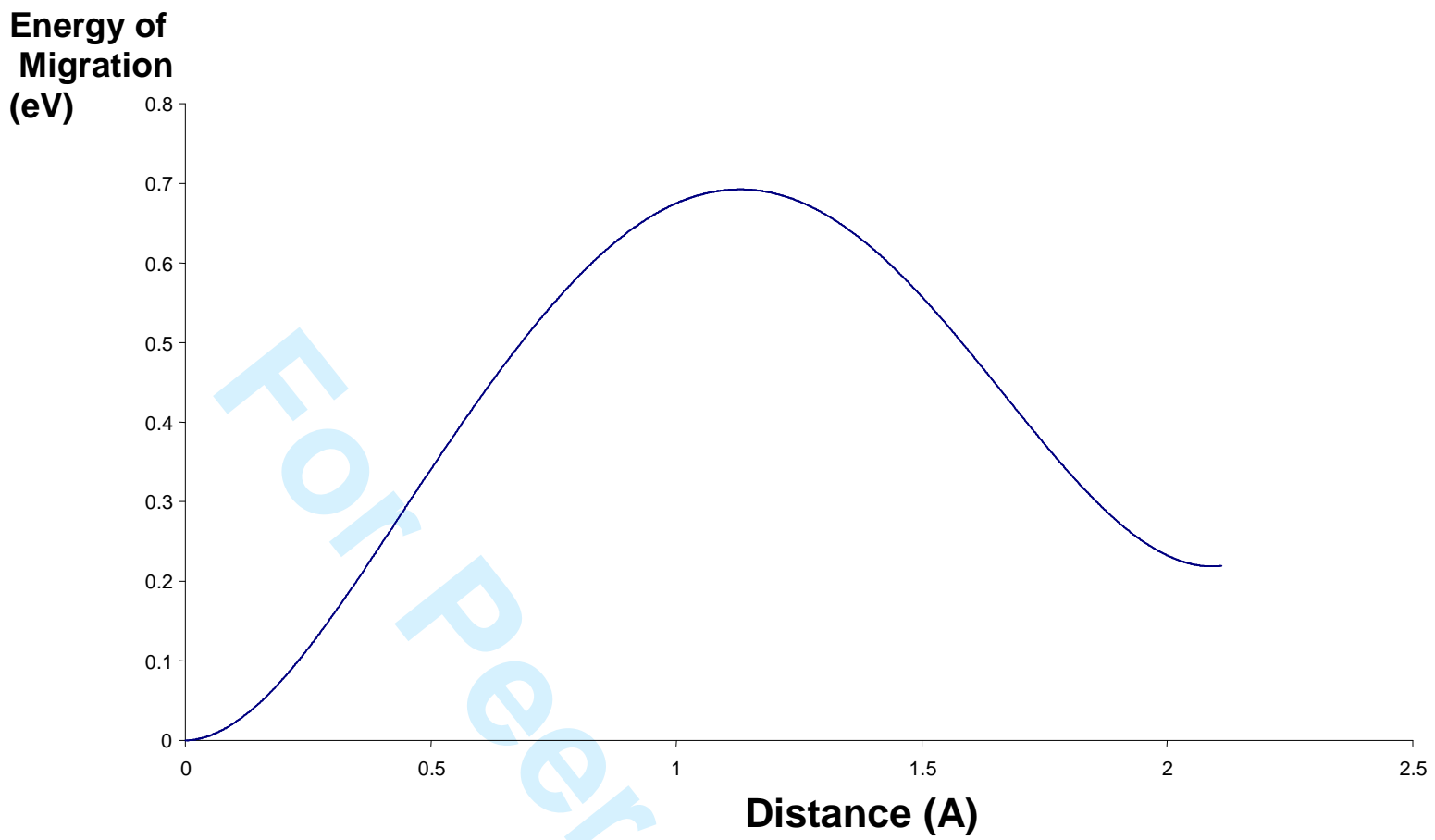

Figure 6: Energy Profile of Oxygen Migration from Bulk to Just Under the $\{111\}$ Surface 


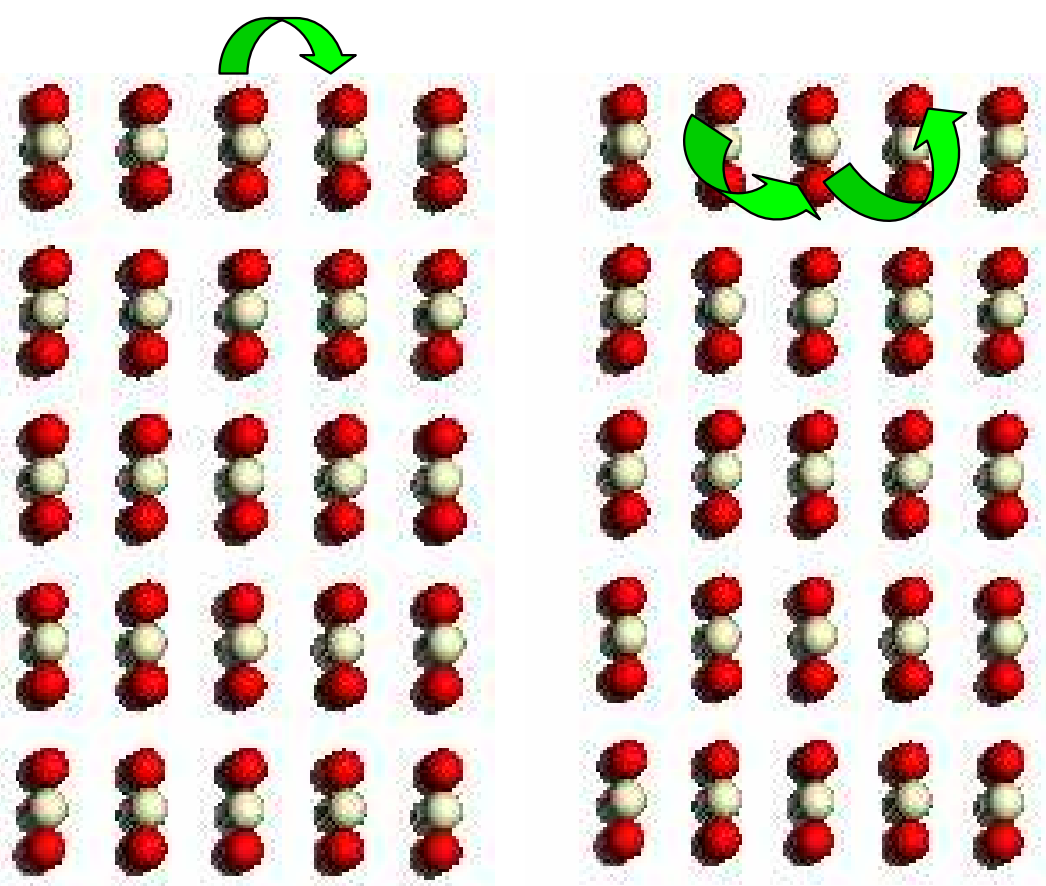

Figure 7 (a) Schematic of oxygen hopping across the surface. $\Delta \mathrm{G} \sim 2.5 \mathrm{eV}$

Figure 7 (b) Schematic of oxygen moving through the surface. $\Delta \mathrm{G} \sim 0.3 \mathrm{eV}$

@ $\mathbf{T}=\mathbf{3 0 0} \mathrm{K}$

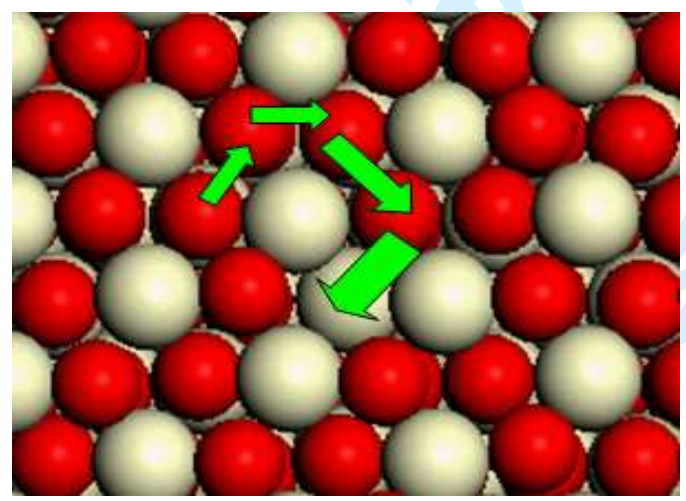

Figure 8: Pathway of Concerted Mechanism 

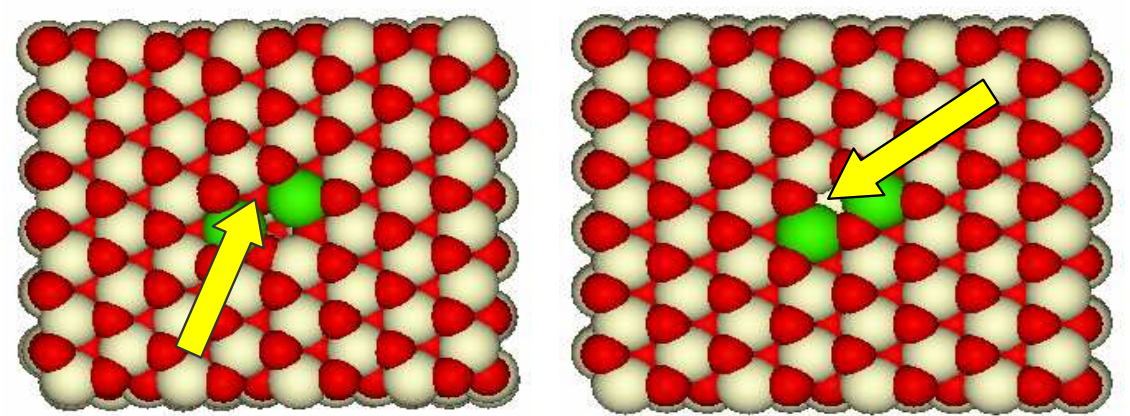

Figure 9a: Before migration and with Surface Vacancy in place. Yellow arrow points to Oxygen that is to be constrained to migrate.

Figure 9b: After Oxygen atom, originally under the surface between $\mathrm{Ce}^{3+}$ ions, has migrated to the surface leaving vacancy just

under the surface and between $\mathrm{Ce}^{3+}$ ions. Green $=\mathrm{Ce}^{3+}$, White $=\mathrm{Ce}^{4+}, \mathrm{Red}=\mathrm{O}^{2-}$ The resultant subsurface vacancy is highlighted by the blue arrow.

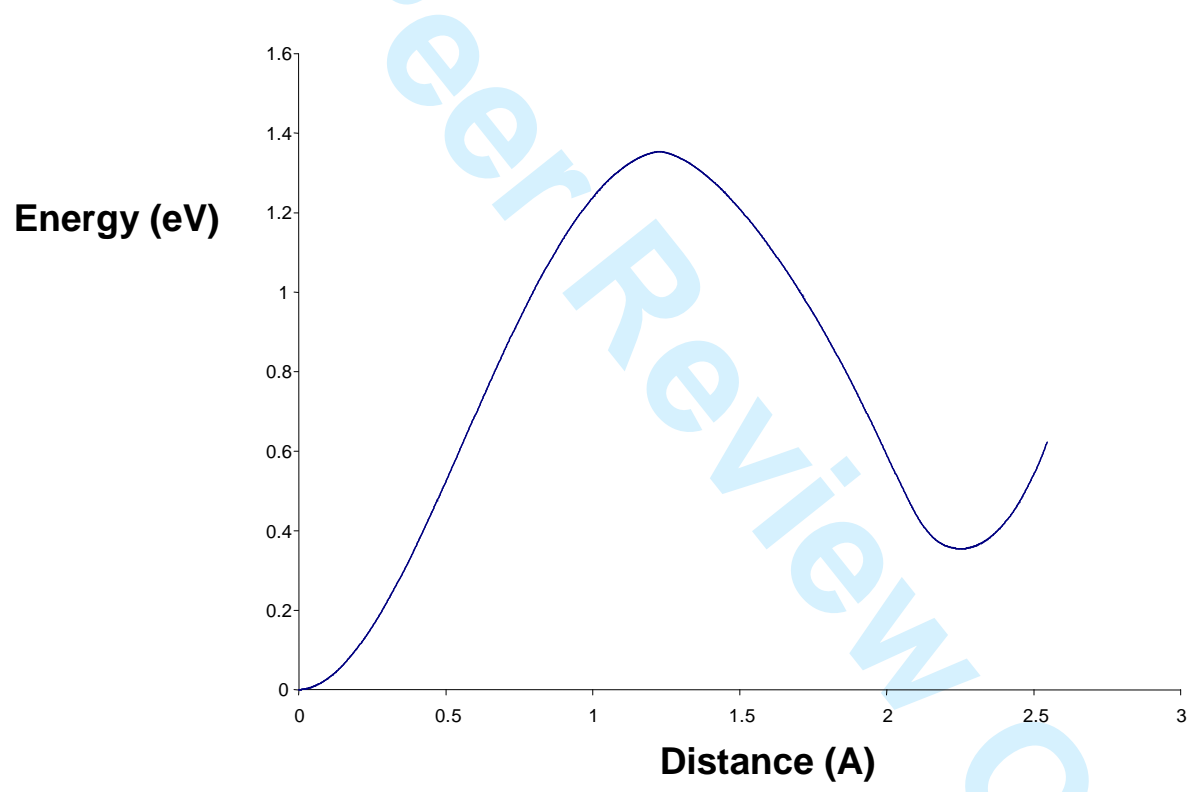

Figure 10: Energy Profile of Oxygen atom migration between $\mathrm{Ce}^{3+}$ ions onto the Surface 

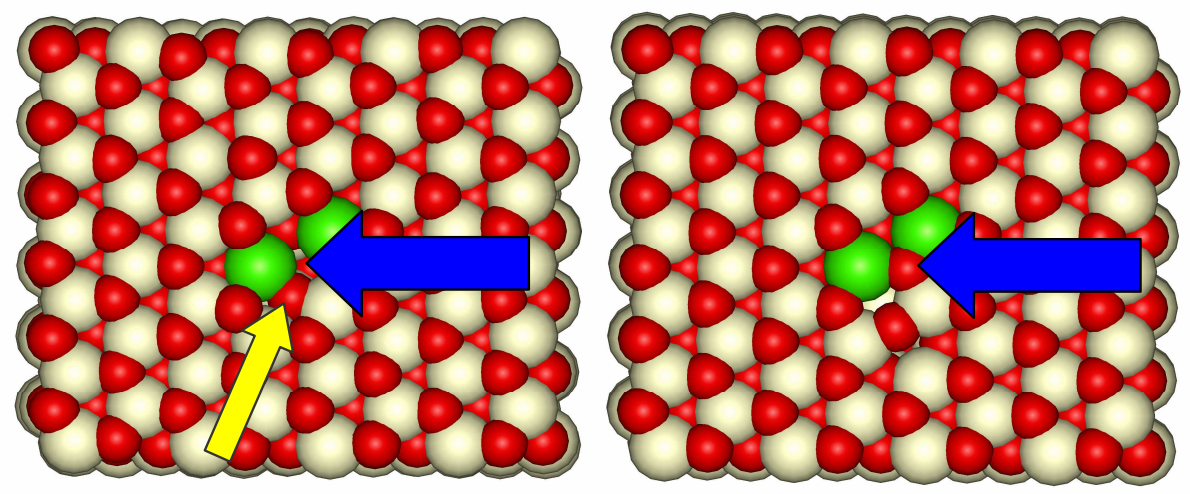

Figure 11a: Before migration and with Surface Vacancy in place (blue arrow). Yellow Arrow points to Oxygen atom to migrate from just beneath the surface.

Figure 11b: After. Oxygen atom, originally under the surface and below the vacancy, has migrated to the surface leaving vacancy just under the surface and below the left $\mathrm{Ce}^{3+}$ ion. Green $=\mathrm{Ce}^{3+}$, White $=\mathrm{Ce}^{4+}, \mathrm{Red}=\mathrm{O}^{2-}$

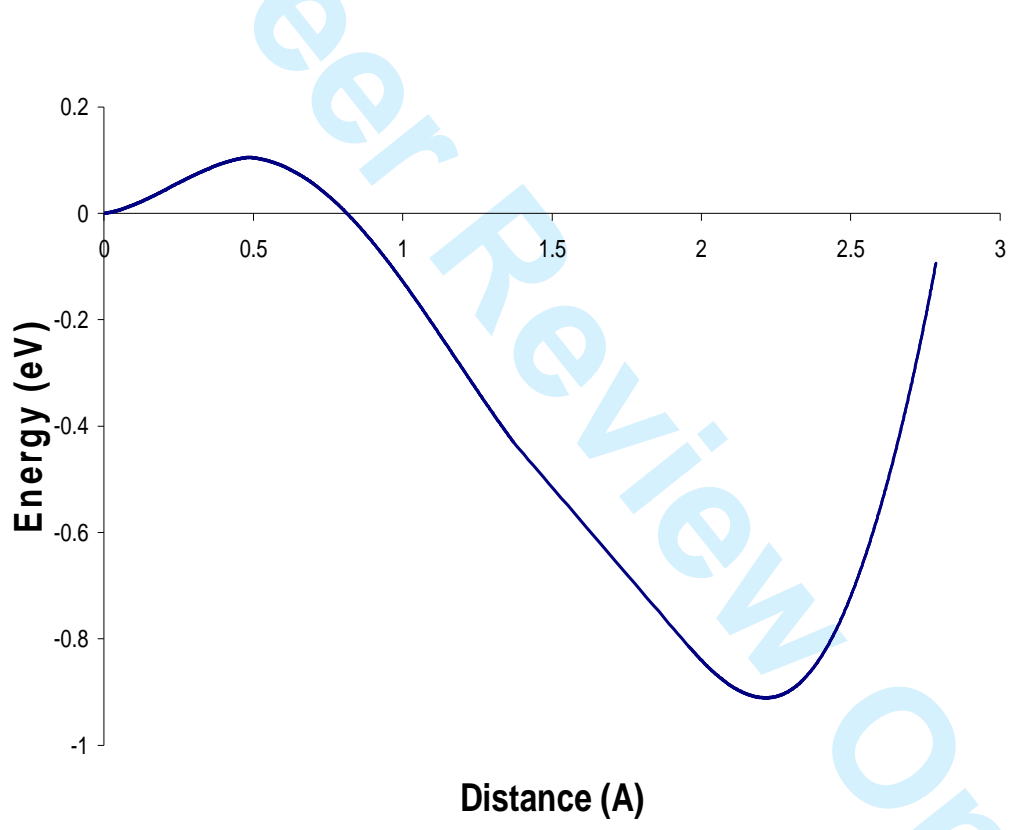

Figure 12: Energy Profile of Oxygen atom migration From under the Left Hand $\mathrm{Ce}^{3+}$ ion into the Surface Vacancy between Compensating $\mathrm{Ce}^{3+}$ ions 

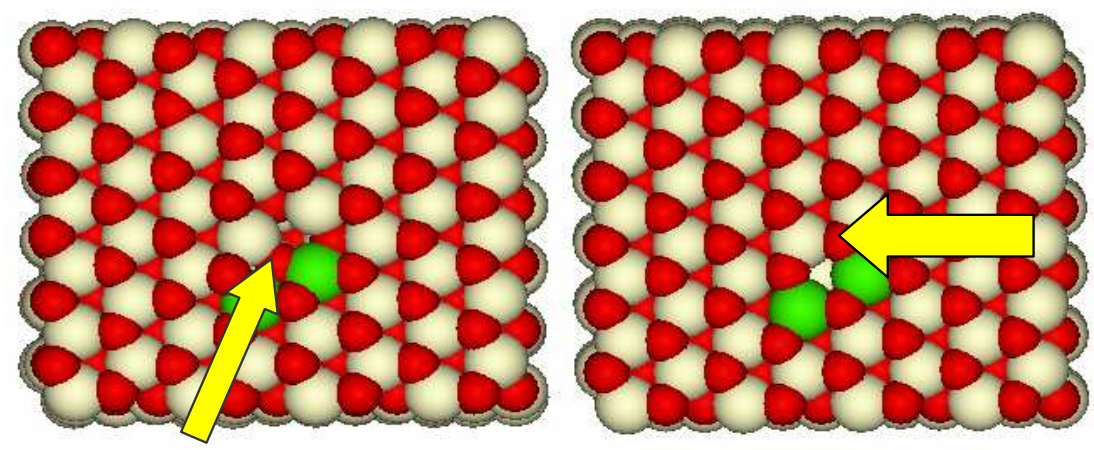

Figure 13a Before migration and with Surface Vacancy in place.

Figure 13b: After. Oxygen atom, originally under the surface between $\mathrm{Ce}^{3+}$ ions, has migrated to the surface leaving vacancy just

under the surface and between $\mathrm{Ce}^{3+}$ ions. Green $=\mathrm{Ce}^{3+}$, White $=\mathrm{Ce}^{4+}$, Red $=\mathrm{O}^{2-}$

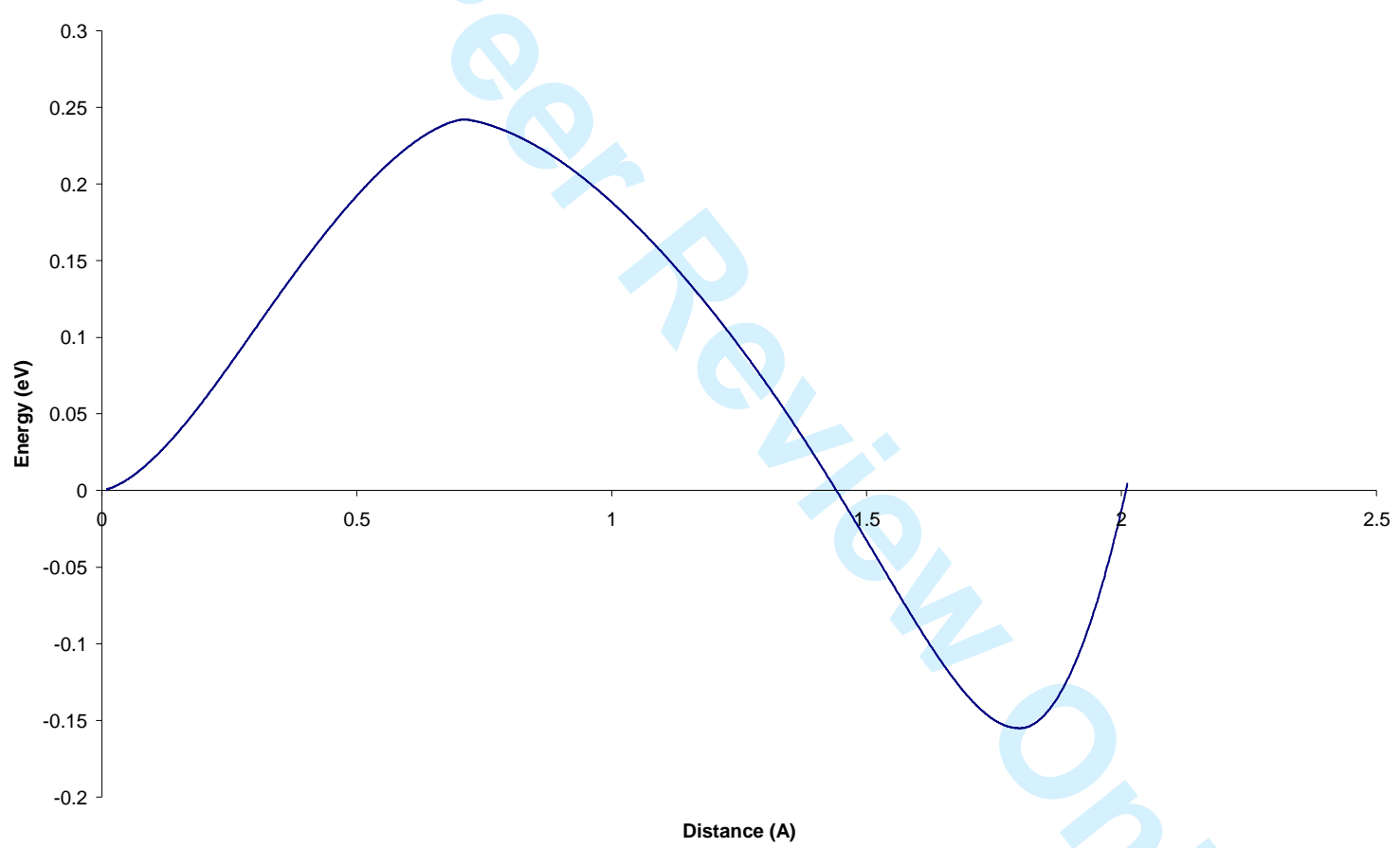

Figure 14: Energy Profile of Oxygen atom migration Away from $\mathrm{Ce}^{3+}$ ions onto the Surface 


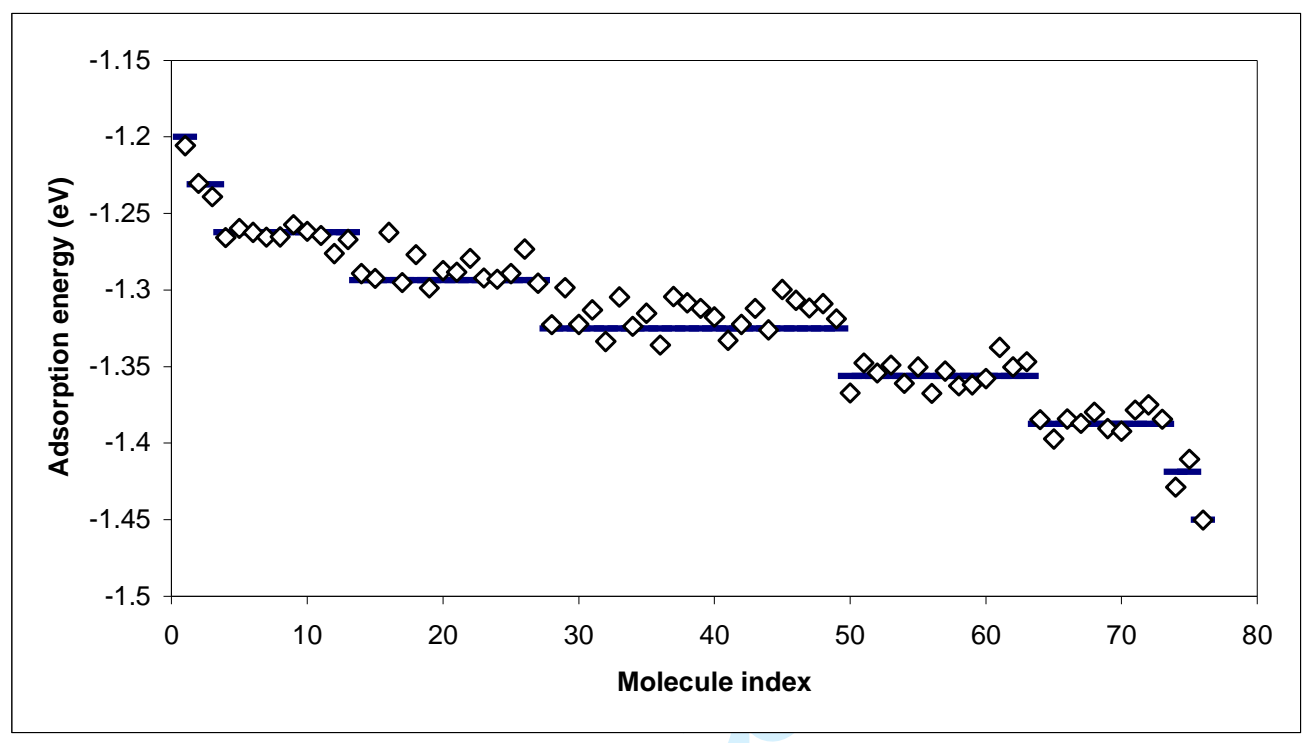

Figure 15: Adsorption energy of the 76 PCDD

http://mc.manuscriptcentral.com/tandf/jenmol 


\section{Page 91 of 158}

1

2

3

4

5

6

7

8

9

10

11

12

13

14

15

16

17

18

19

20

21

22

23

24

25

26

27

28

29

30

31

32

33

34

35

36

37

38

39

40

41

42

43

44

45

46

47

48

49

50

51

52

53

54

55

56

57

58

59

60

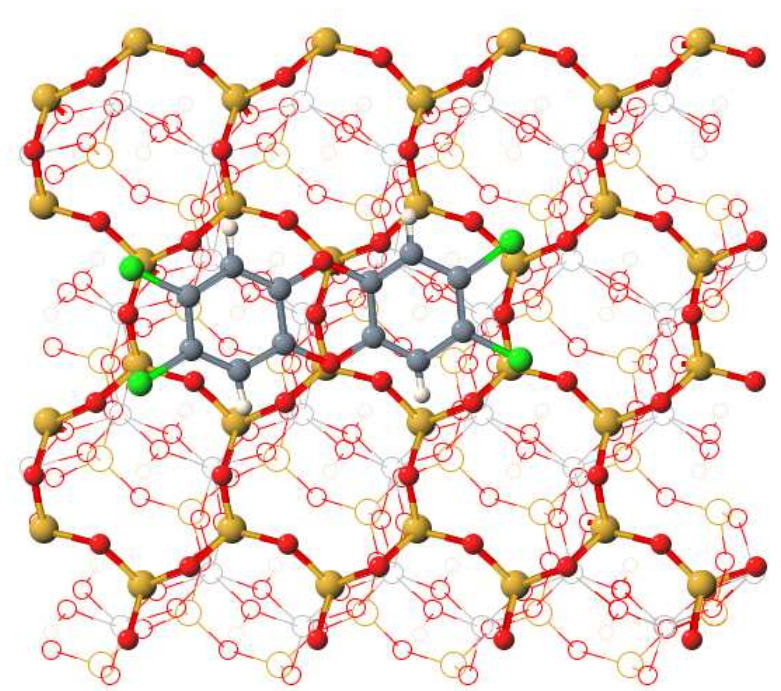

Figure 16: Adsorption position of a PCDD congener. The surface is parallel to the page. The shaded atoms represent the lower layers. Surface: Si Large light grey, O small dark grey. Molecule: H white, $\mathrm{Cl}$ light grey, $\mathrm{C}$ grey, $\mathrm{O}$ dark grey 
2

3

4

5

6

7

8

9

10

11

12

13

14

15

16

17

18

19

20

21

22

23

24

25

26

27

28

29

30

31

32

33

34

35

36

37

38

39

40

41

42

43

44

45

46

47

48

49

50

51

52

53

54

55

56

57

58

59

60

Potential energy against time of an MD simulation of $8 \mathrm{MgO}$ nanoparticles

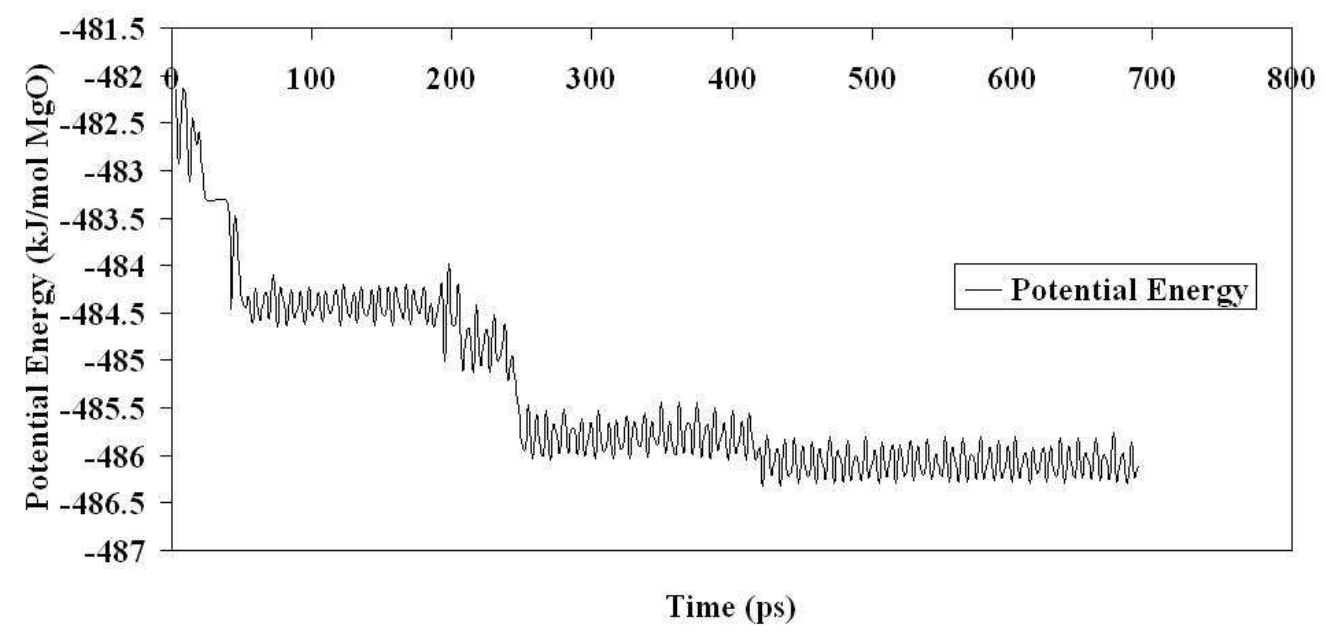

Figure 18: Potential energy per magnesium ion against time of an MD simulation of $8 \mathrm{MgO}$ nanoparticles
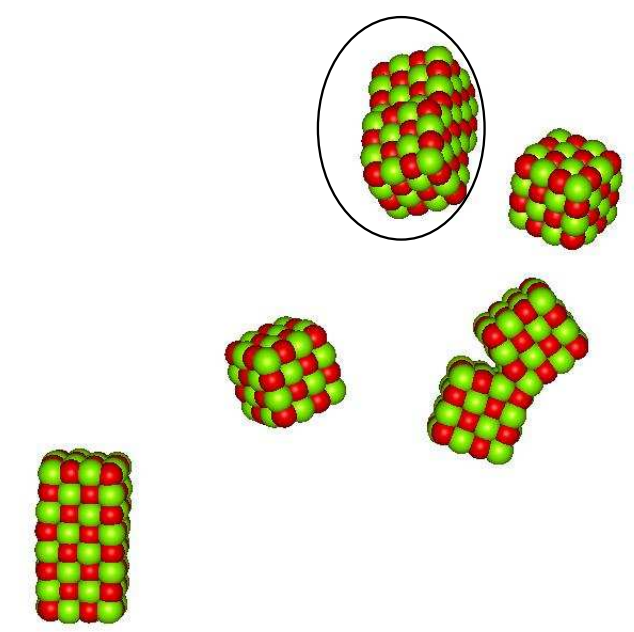

Figure 19: A snapshot of the MD simulation at 30ps for the MgO system 
Figure20: A snapshot of the MD simulation at 60ps for the MgO system
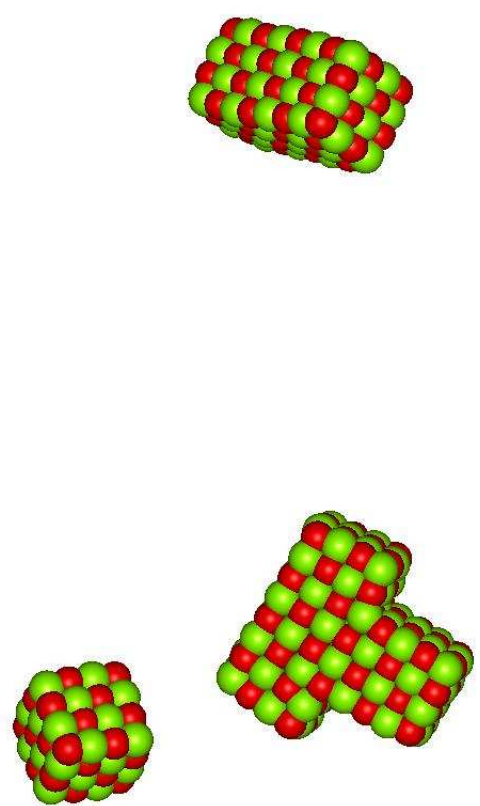

Figure 21: A snapshot of the MD simulation at 220ps for the MgO system 
Figure 22: A snapshot of the MD simulation at 275ps for the MgO system

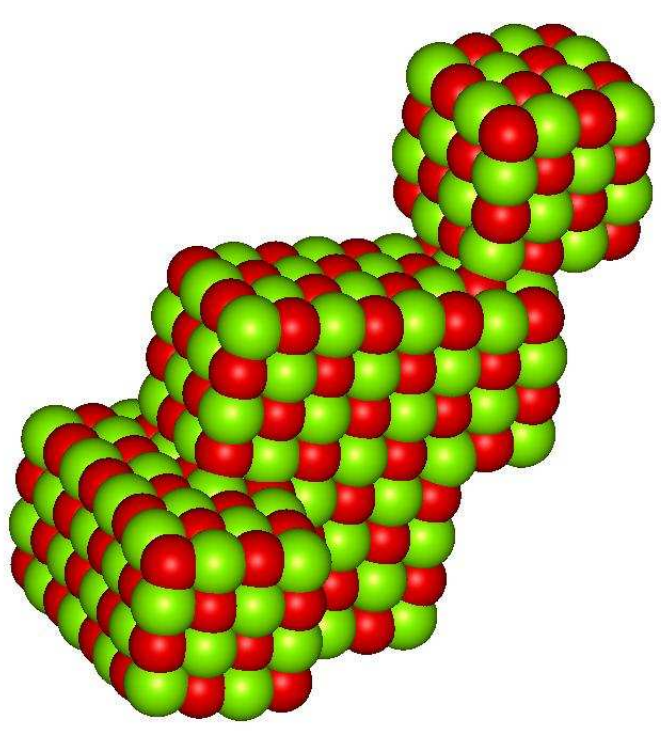

Figure 23: Final configuration of the aggregated MgO nanoparticles 
Figure 8a: Mg-O RDF of the Aggregated MgO Nanoparticle

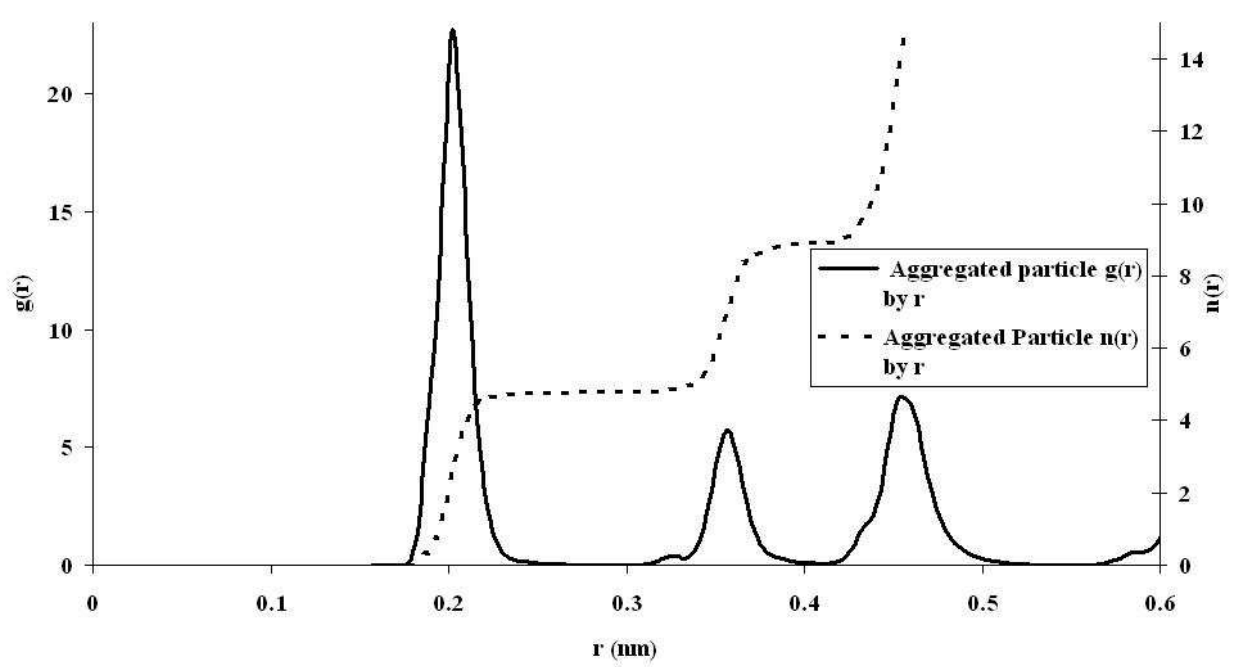

Figure 8b: Mg-O RDF of Bulk Magnesium Oxide

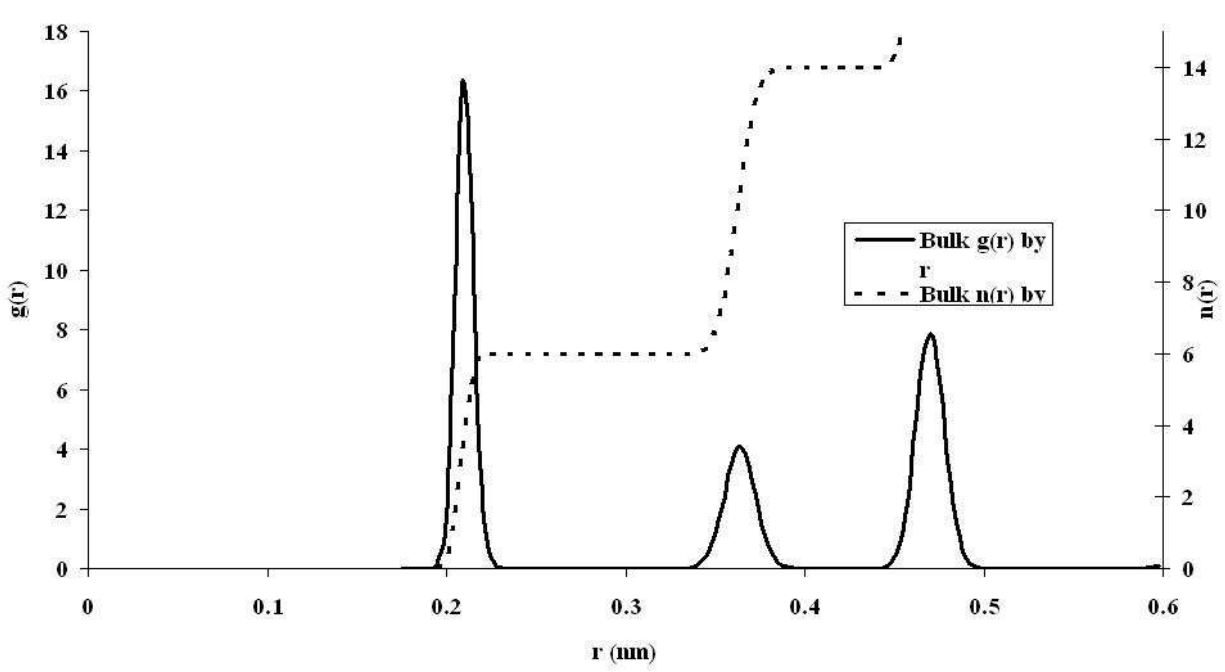

Figure 24: RDF of MgO (a) aggregated nanoparticle and (b) Bulk 


\section{Page 97 of 158}

1

2

3

4

5

6

7

8

9

10

11

12

13

14

15

16

17

18

19

20

21

22

23

24

25

26

27

28

29

30

31

32

33

34

35

36

37

38

39

40

41

42

43

44

45

46

47

48

49

50

51

52

53

54

55

56

57

58

59

60
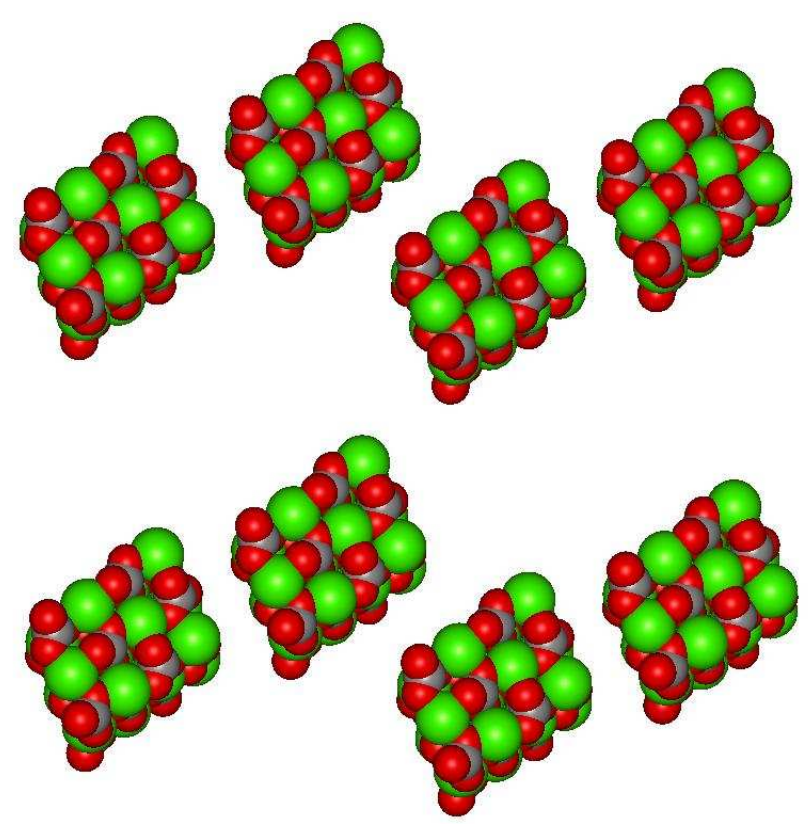

Figure 25: Initial configuration of calcite nanoparticles for MD simulation

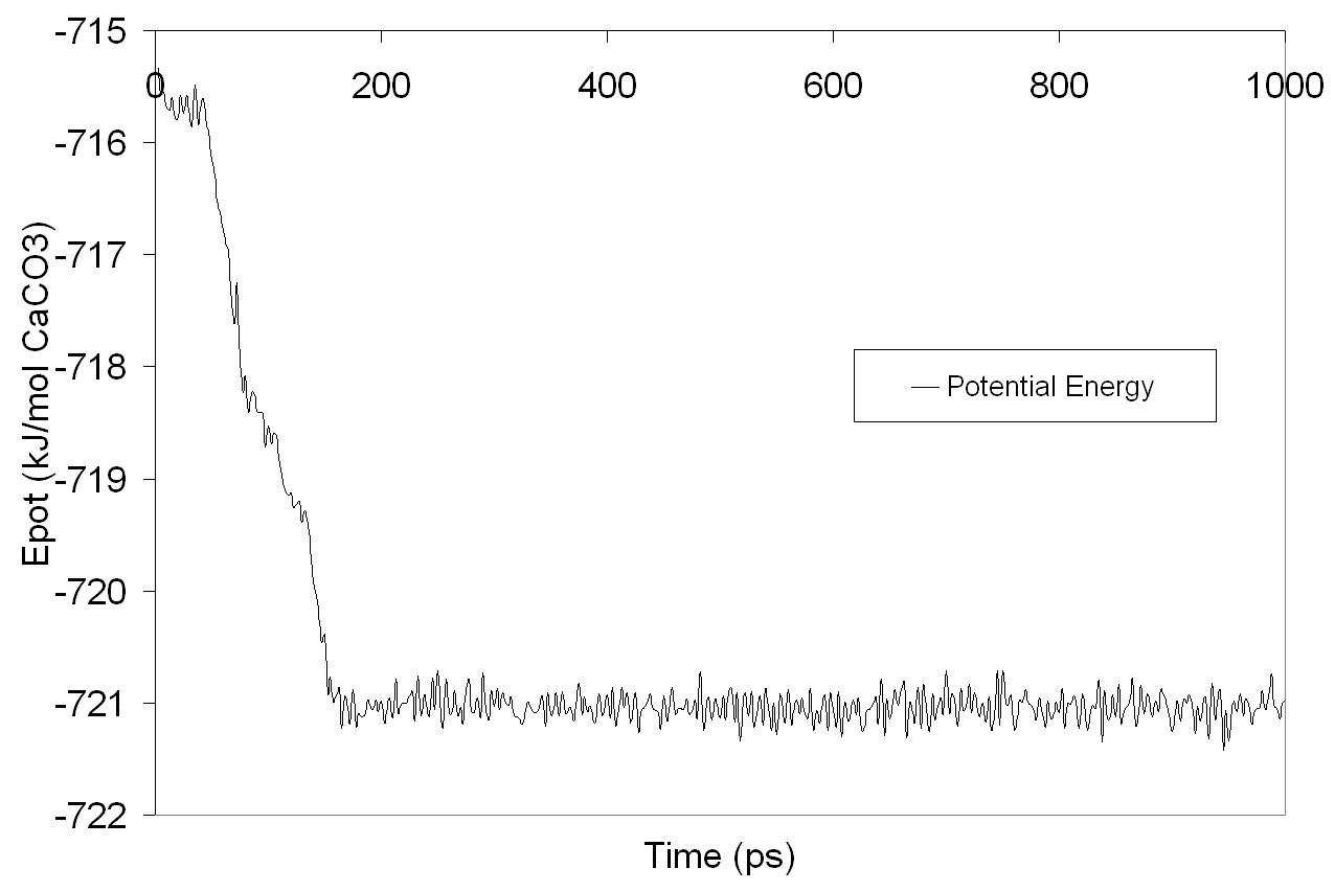

Figure 26: Potential energy per calcium ion against time of an MD simulation of 8 calcite nanoparticles 
Figure 27: A snapshot of the MD simulation at 50ps for the calcite $1.6 \mathrm{~nm}$ system 


\section{Page 99 of 158}

1
2
3
4
5
6
7
8
9
10
11
12
13
14
15
16
17
18
19
20
21
22
23
24
25
26
27
28
29
30
31
32
33
34
35
36
37
38
39
40
41
42
43
44
45
46
47
48
49
50
51
52
53
54
55
56
57
58
59
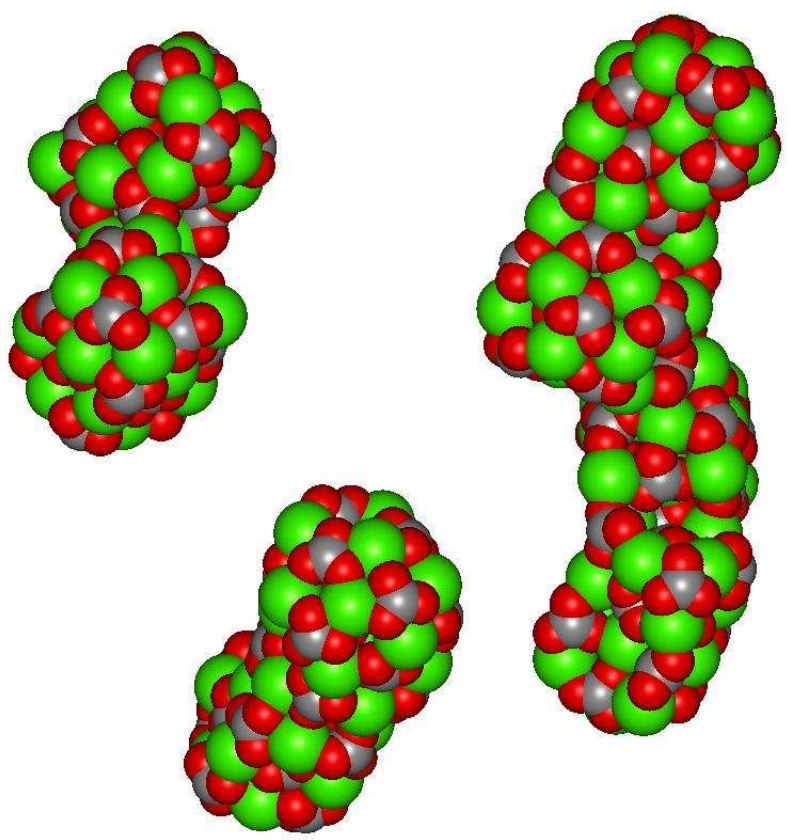

Figure 28: A snapshot of the MD simulation at 87ps for the calcite 1.6nm system

29

32

33

34

35

36

37

39

40

41

42

43

44

46

47

48

49

50

52 


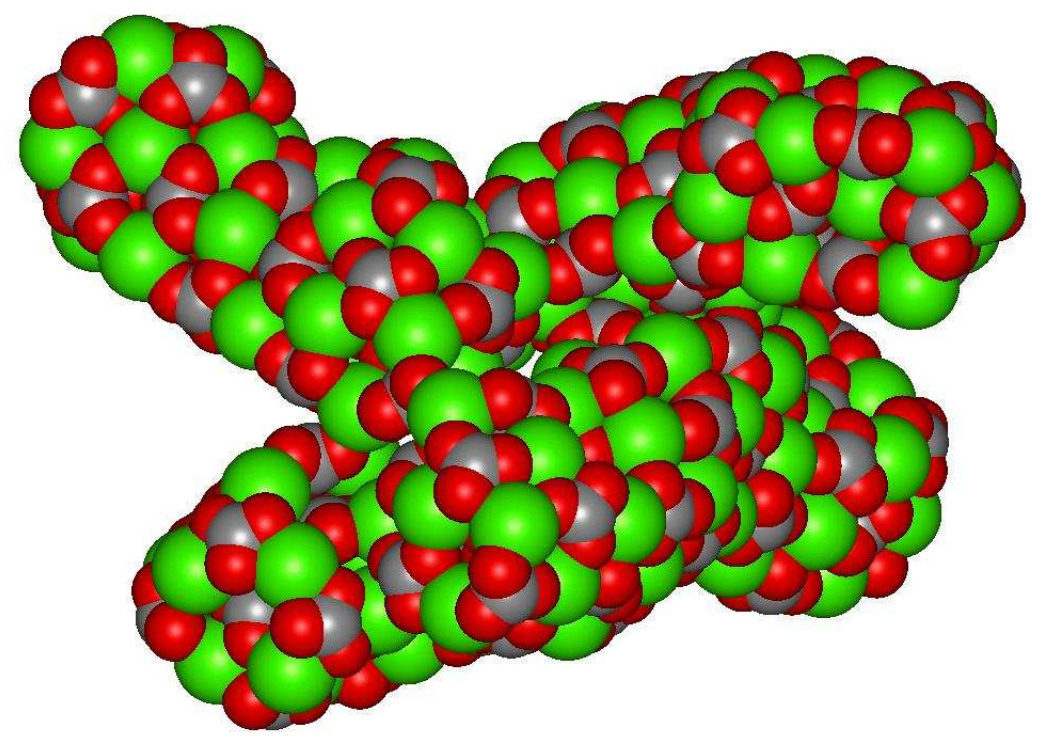

Figure 29: snapshot of the MD simulation at 140ps for the calcite 1.6nm system

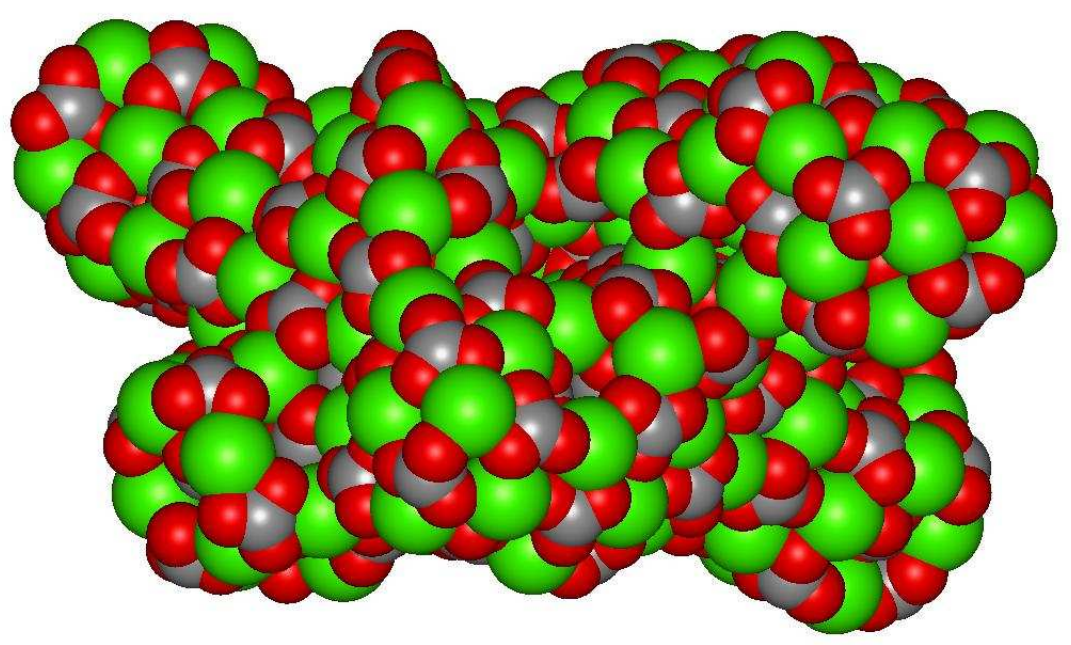

Figure 30: Final configuration of the aggregated calcite nanoparticles 
Figure 15a: Ca-O RDF of the Aggregated Calcite Nanoparticle

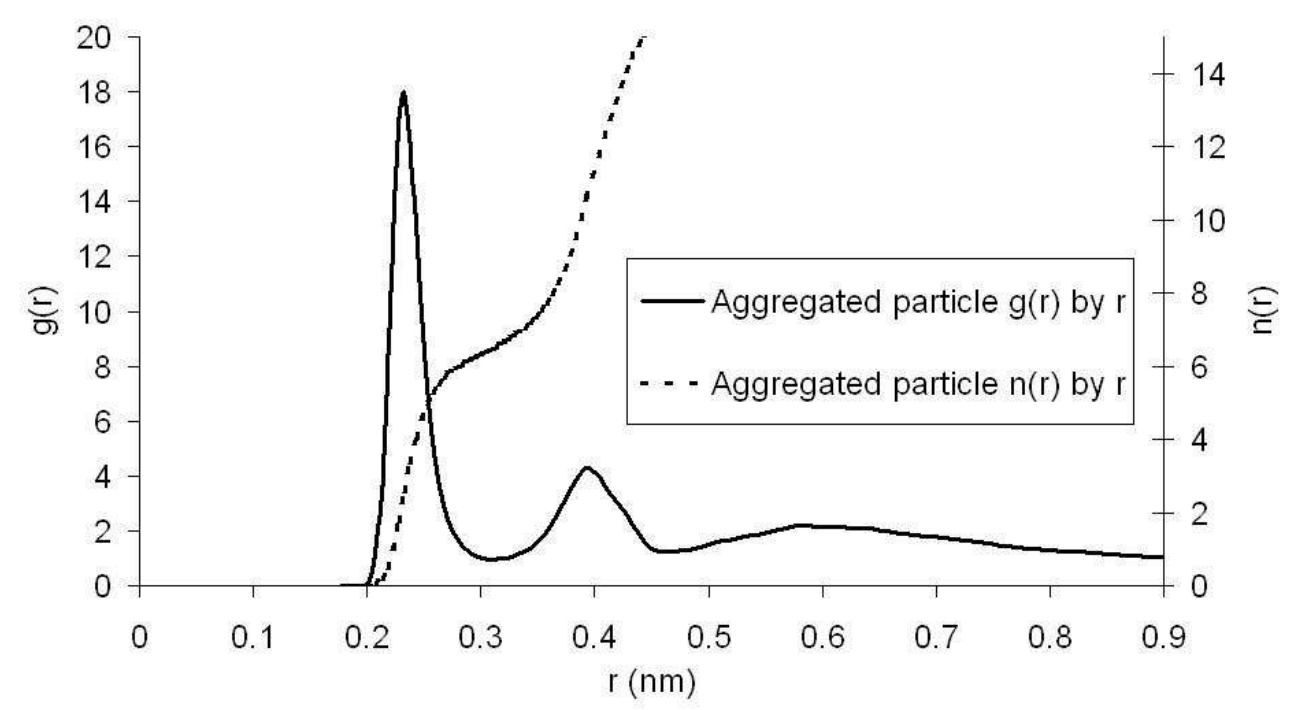

Figure 15b: Ca-O RDF in Bulk Calcite

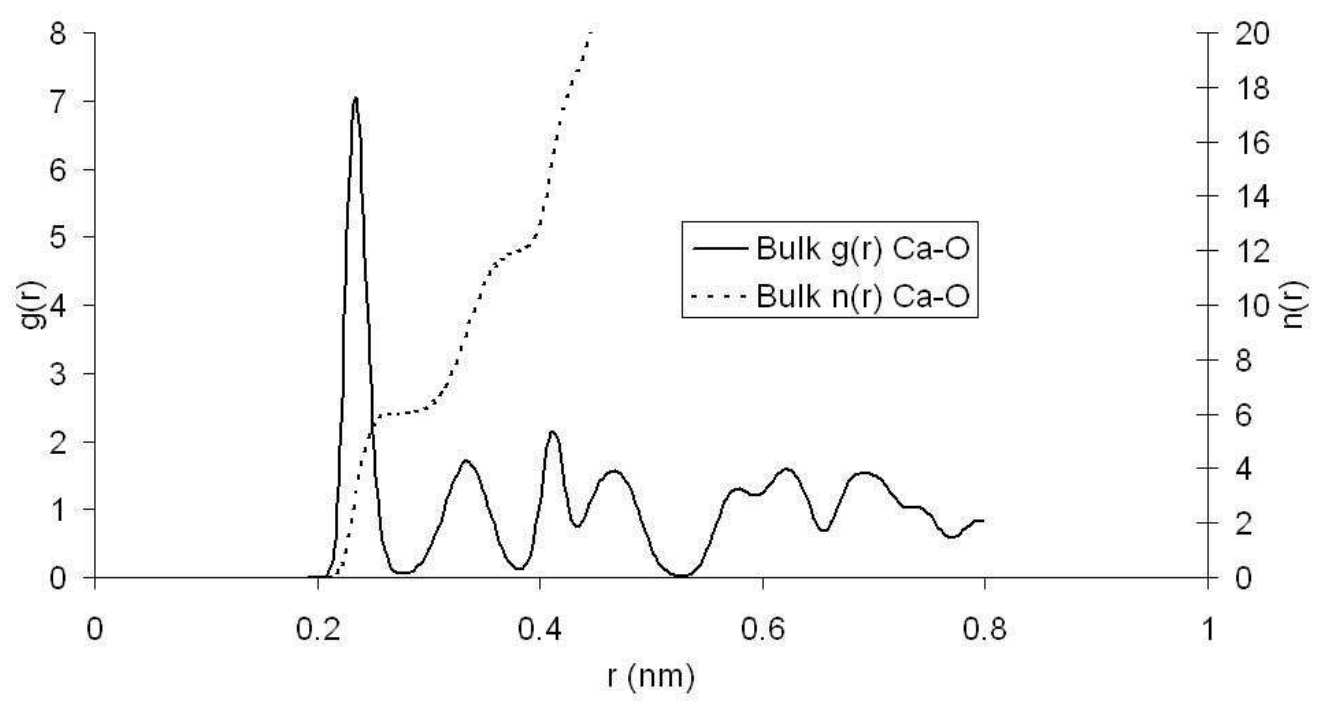

Figure 31: RDF of the (a) aggregated calcite nanoparticles and (b) bulk calcite 


\begin{tabular}{lc}
\hline \multicolumn{1}{c}{ Specie } & Charge \\
\hline $\mathrm{H}$ & .20 \\
$\mathrm{Cl}$ & -.03 \\
$\mathrm{O}$ & -.50 \\
$\mathrm{C}(\mathrm{H}$ bonded $)$ & -.20 \\
$\mathrm{C}(\mathrm{Cl}$ bonded $)$ & .03 \\
$\mathrm{C}(\mathrm{O}$ bonded $)$ & .25 \\
\hline \hline
\end{tabular}

Table 1: Charge model (in e)

13
14
15
16
17
18
19
20
21
22
23
24
25
26
27
28
29
30
31
32
33
34
35
36
37
38
39
40
41
42
43
44
45
46
47
48
49
50
51
52
53
54
55
56
57
58
59
60

http://mc.manuscriptcentral.com/tandf/jenmol 


\section{Page 28: [1] Deleted}

1. White, T.O., R.P. Bruin, J. Wakelin, C. Chapman, D. Osborn, P. Murray-Rust, E. Artacho, M.T. Dove, and M. Calleja. eScience methods for the combinatorial chemistry problem of adsorption of pollutant organic molecules on mineral surfaces. Proceedings of the UK e-Science All Hands Meeting 2005. (2005)

2. Deluga, G.A., J.R. Salge, L.D. Schmidt, and X.E. Verykios. Renewable hydrogen from ethanol by autothermal reforming. Science. 303. 5660. (2004)

3. Otsuka, K., T. Ushiyama, and I. Yamanaka. Partial Oxidation of Methane Using the Redox of Cerium Oxide. Chemistry Letters. 9. (1993)

4. Trovarelli, Catalysis by Ceria and Related Materials. 2002, London: Imperial College Press.

5. Park, S.D., J.M. Vohs, and R.J. Gorte. Direct oxidation of hydrocarbons in a solid-oxide fuel cell. Nature. 404. 6775. (2000)

6. Watson, G.W., E.T. Kelsey, N.H. deLeeuw, D.J. Harris, and S.C. Parker. Atomistic simulation of dislocations, surfaces and interfaces in MgO. Journal of the Chemical Society-Faraday Transactions. 92. 3. (1996)

7. Balducci, G., J. Kaspar, P. Fornasiero, M. Graziani, M.S. Islam, and J.D. Gale. Computer simulation studies of bulk reduction and oxygen migration in $\mathrm{CeO} 2-$ $\mathrm{ZrO} 2$ solid solutions. Journal of Physical Chemistry B. 101. 10. (1997)

8. Sayle, T.X.T., S.C. Parker, and C.R.A. Catlow. Surface Segregation of Metal-Ions in Cerium Dioxide. Journal of Physical Chemistry. 98. 51. (1994)

9. Balducci, G., M.S. Islam, J. Kaspar, P. Fornasiero, and M. Graziani. Bulk reduction and oxygen migration in the ceria-based oxides. Chemistry of Materials. 12. 3. (2000)

10. Lewis, G.V. and C.R.A. Catlow. Potential Models for Ionic Oxides. Journal of Physics C-Solid State Physics. 18. 6. (1985)

11. Dick, A.W. and B.G. Overhauser. Theory of dielectric constants of alkali halide crystals. Physical Reviews. 112. 1. (1958)

12. Dauberosguthorpe, P., V.A. Roberts, D.J. Osguthorpe, J. Wolff, M. Genest, and A.T. Hagler. Structure and Energetics of Ligand-Binding to Proteins Escherichia-Coli Dihydrofolate Reductase Trimethoprim, a Drug-Receptor System. Proteins-Structure Function and Genetics. 4. 1. (1988)

13. Frisch, M.J., G.W. Trucks, H.B. Schlegel, G.E. Scuseria, M.A. Robb, J.R. Cheeseman, J. Montgomery, J. A., T. Vreven, K.N. Kudin, J.C. Burant, J.M. Millam, S.S. Iyengar, J. Tomasi, V. Barone, B. Mennucci, M. Cossi, G. Scalmani, N. Rega, G.A. Petersson, H. Nakatsuji, M. Hada, M. Ehara, K. Toyota, R. Fukuda, J. Hasegawa, M. Ishida, T. Nakajima, Y. Honda, O. Kitao, H. Nakai, M. Klene, X. Li, J.E. Knox, H.P. Hratchian, J.B. Cross, V. Bakken, C. Adamo, J. Jaramillo, R. Gomperts, R.E. Stratmann, O. Yazyev, A.J. Austin, R. Cammi, C. Pomelli, J.W. Ochterski, P.Y. Ayala, K. Morokuma, G.A. Voth, P. Salvador, J.J. Dannenberg, V.G. Zakrzewski, S. Dapprich, A.D. Daniels, M.C. Strain, O. Farkas, D.K. Malick, A.D. Rabuck, K. Raghavachari, J.B. Foresman, J.V. Ortiz, Q. Cui, A.G. Baboul, S. Clifford, J. Cioslowski, B.B. Stefanov, G. Liu, A. Liashenko, P. Piskorz, I. Komaromi, R.L. Martin, D.J. Fox, T. Keith, M.A. AlLaham, C.Y. Peng, A. Nanayakkara, M. Challacombe, P.M.W. Gill, B. Johnson, W. Chen, M.W. Wong, C. Gonzalez, and J.A. Pople, Gaussian 03, Revision C.02. 2004, Gaussian, Inc.: Wallingford CT. 
14. Zhukova, A.I., S.V. Bondarenko, and Y.I. Tarasevich. Study of Selectivity of Natural Sorbents with Respect to Hydrocarbons. Ukrainskii Khimicheskii Zhurnal. 42. 7. (1976)

15. Keldsen, G.L., J.B. Nicholas, K.A. Carrado, and R.E. Winans. Molecular Modeling of the Enthalpies of Adsorption of Hydrocarbons on Smectite Clay. Journal of Physical Chemistry. 98. 1. (1994)

16. Smith, W. and T.R. Forester. DL_POLY_2.0: A general-purpose parallel molecular dynamics simulation package. Journal of Molecular Graphics. 14. 3. (1996)

17. Gale, J.D. and A.L. Rohl. The General Utility Lattice Program (GULP). Molecular Simulation. 29. 5. (2003)

18. Spano, E., S. Hamad, and C.R.A. Catlow. Computational evidence of bubble ZnS clusters. Journal of Physical Chemistry B. 107. 38. (2003)

19. Spano, E., S. Hamad, and C.R.A. Catlow. ZnS bubble clusters with onion-like structures. Chemical Communications. 7. (2004)

20. Zhang, H.Z., F. Huang, B. Gilbert, and J.F. Banfield. Molecular dynamics simulations, thermodynamic analysis, and experimental study of phase stability of zinc sulfide nanoparticles. Journal of Physical Chemistry B. 107. 47. (2003)

21. Zhang, H.Z., B. Gilbert, F. Huang, and J.F. Banfield. Water-driven structure transformation in nanoparticles at room temperature. Nature. 424. 6952. (2003)

22. Zhang, H.Z. and J.F. Banfield. Aggregation, coarsening, and phase transformation in $\mathrm{ZnS}$ nanoparticles studied by molecular dynamics simulations. Nano Letters. 4. 4. (2004)

23. Lewis, G.V. and C.R.A. Catlow. Potential model for ionic oxides. Journal of Physical Chemistry: Solid State Physics. 18. (1985)

24. de Leeuw, N.H. and S.C. Parker. Molecular-dynamics simulation of MgO surfaces in liquid water using a shell-model potential for water. Physical Review B. 58. 20. (1998)

25. Harris, D.J., J.H. Harding, and S.C. Parker. Simulations of surfaces and interfaces in MgO. Radiation Effects and Defects in Solids. 151. 1-4. (1999)

26. Sayle, D.C., J.A. Doig, S.A. Maicaneanu, and G.W. Watson. Atomistic structure of oxide nanoparticles supported on an oxide substrate. Physical Review B. 65. 24. (2002)

27. Pavese, A., M. Catti, S.C. Parker, and A. Wall. Modelling of the thermal dependence of structural and elastic properties of calcite, $\mathrm{CaCO}_{3}$. Physics and Chemistry of Minerals. 23. 2. (1996)

28. Kerisit, S. and S.C. Parker. Free energy of adsorption of water and calcium on the \{10(1)over-bar-4\} calcite surface. Chemical Communications. 1. (2004)

29. Kerisit, S., D.J. Cooke, D. Spagnoli, and S.C. Parker. Molecular dynamics simulations of the interactions between water and inorganic solids. Journal of Materials Chemistry. 15. 14. (2005)

30. Bragg, W.L. Crystal structure. Nature. 105. (1920)

31. Tsirel'son, V.G.A., A.S.;Abramov, Yu.A.;Belokoneva, E.L.;Kitaneh, R.;Feil, D. X-ray and electron diffraction study of Mg O. Acta Crystallographica B. 54. (1998) 
32. Maslen, E.N.S.t., V.A.;Strel'tsova, N.R. X-ray study of the electron density in calcite, CaCO3. Acta Crystallographica B. 49. (1993)

33. Wyckoff, R.W.G. The crystal structures of some carbonates of the calcite group. American Journal of Science, Serie 50. (1920)

34. Addadi, L., S. Raz, and S. Weiner. Taking advantage of disorder: Amorphous calcium carbonate and its roles in biomineralization. Advanced Materials. 15. 12. (2003)

35. Faatz, M., F. Grohn, and G. Wegner. Amorphous calcium carbonate: Synthesis and potential intermediate in biomineralization. Advanced Materials. 16. 12. (2004)

36. Weiner, S., Y. Levi-Kalisman, S. Raz, and L. Addadi. Biologically formed amorphous calcium carbonate. Connective Tissue Research. 44. (2003)

37. Xu, X.R., J.T. Han, and K. Cho. Formation of amorphous calcium carbonate thin films and their role in biomineralization. Chemistry of Materials. 16. 9. (2004)

38. Q. H. Zeng, A. B. Yu, G. Q. Lu, and R. K. Standish. Molecular Dynamics Simulation of Organic-InorganicNanocomposites: Layering Behavior and Interlayer Structure of Organoclays. Chemistry of Materials. Mater., 15 (25), $4732-4738,2003$.

$39 \mathrm{Yu}, \mathrm{CH}$, Molecular dynamics simulations of adsorption of organic compounds at the clay mineral/aqueous solution interface Structural chemistry [1040-0400] 14, 2 pg:175 -185 (2003).

$40 \mathrm{Yu}, \mathrm{CH}$, Newton, SQ, Norman, MA, et al. Molecular dynamics simulations of adsorption of organic compounds at the clay mineral/aqueous solution interface STRUCT CHEM 14 (2): 175-185 APR (2003)

$41 \quad$ Neal T. Skipper a, $\square$, Peter A. Lock a, James O. Titiloye b, Jan Swenson c,

Zakaria A. Mirza a, W. Spencer Howells d, Felix Fernandez-Alonso d. The structure and dynamics of 2-dimensional fluids in swelling clays. Chemical Geology $230182-$ 196 (2006)

42 Thompson, MVR, Greathouse, JA Computer simulations of smectite clay minerals. ABSTR PAP AM CHEM S 227: U446-U446 291-CHED Part 1 MAR 28 (2004)

43 Michael Nolan, Stephen C. Parker, Graeme W. Watson. The electronic structure of oxygen vacancy defects at the low index surfaces of ceria Surface Science 595 (2005) 223-232.

44 Michael Nolan, Sonja Grigoleit, Dean C. Sayle, Stephen C. Parker and Graeme W. Watson Density functional theory studies of the structure and electronic structure of pure and defective low index surfaces of ceria Surface Science, Volume 576, Issues 1-3, 10 February 2005, Pages 217-229

45 Michael Nolan, Stephen C. Parker, and Graeme W. Watson Reduction of $\mathrm{NO}_{2}$ on Ceria Surfaces J. Phys. Chem. B; 2006; 110(5) pp 2256 - 2262

$46 \mathrm{CeO}_{2}$ catalysed conversion of $\mathrm{CO}, \mathrm{NO}_{2}$ and $\mathrm{NO}$ from first principles energetics Michael Nolan, Stephen C. Parker and Graeme W. Watson Phys. Chem. Chem. Phys., 2006, 8, 216 - 218

47 Modelling inorganic solids and their interfaces: A combined approach of atomistic and electronic structure simulation techniques S.C. Parker, S. Kerisit, A. Marmier, S. Grigoleit,G.W. Watson Faraday Discuss. 124, 155-170 (2003).

48 Free Energy of Adsorption of Water and Metal Ions on the $\{1014\}$ Calcite Surface 
Sebastien Kerisit and Stephen C. Parker J. Am. Chem. Soc.; 2004; 126(32) pp 10152 10161

49 Novel exchange mechanisms in the surface diffusion of oxides D.J. Harris, M.Y. Lavrentiev, J.H. Harding, N.L. Allan and J.A. Purton, J. Phys. Cond. Mat. 16 (2004) L187-L192 


\title{
Application of Molecular Dynamics DL_POLY Codes to
}

\author{
Interfaces of Inorganic Materials
}

Paul Martin, Dino Spagnoli, Arnaud Marmier, Stephen C. Parker.

Department of Chemistry, University of Bath, Claverton Down, Bath, UK

BA2 7AY. E-mail: s.c.parker@bath.ac.uk: Fax: +44 1225 386231, Tel: +44

1225386505

Dr. Dean C. Sayle Defence Academy of the United Kingdom, Cranfield

University Shrivenham, Swindon United Kingdom SN6 8LA. E-mail:

d.c.sayle@cranfield.ac.uk Fax:+44 (0)1793 785772 , Tel:+44 (0)1793

785264

Dr. Graeme Watson School of Chemistry, University of Dublin, Trinity

College, Dublin 2. E-mail: watsong@ @tcd.ie Fax:+353 (0)1 6712826 Tel:

$+353(0) 16081357$

\begin{abstract}
Three recent applications of the DL_POLY molecular dynamics code are described, which demonstrate the flexibility and viability of the code for extending our understanding of the structure, stability and reactivity of ceramics and minerals at the atomic level. The first is an investigation into differences in oxygen atom mobility in bulk and at the most stable $\{111\}$ surface of ceria. The results show enhanced surface transport but that it is via subsurface oxygen. Secondly, we investigate how polychlorodibenzo-pdioxins (PCDDs) molecules might adsorb on clay surfaces. The resulting adsorption energies show a clear relationship with chlorine content of the molecule. Finally, we apply DL_POLY to comparing the aggregation of magnesium oxide and calcium carbonate nanoparticles. We find that very small calcium
\end{abstract}


carbonate nanoparticles are amorphous and their aggregation shows no preferred orientation in contrast to magnesium oxide, which remain highly crystalline and combine in a highly structural specific way.

\section{Introduction}

The aim of this paper is to describe our current work using DL_POLY to model the structures and energetics of inorganic solids, with particular emphasis on surfaces and nanostructures. We illustrate the scope of DL_POLY to modelling surfaces and interfaces by considering three recent examples. The first example describes our application of DL_POLY to explore the stability and transport of surface defects, oxygen vacancies on the most stable surface of ceria. Understanding the factors controlling the formation of surface defects is of importance for understanding surface properties. As it is the surface defect that is often the site of chemical activity. Surface defects can also include adsorbed molecules and in our second example we are following the DFT work by the Cambridge group [1] by applying DL_POLY to help our understanding of adsorption processes. Nanoparticles are of great scientific interest as they are effectively a bridge between bulk materials and atomic or molecular structures. The interesting and sometimes unexpected properties of nanoparticles are partly due to their large number of surface sites aspects. The percentage of atoms at the surface of a material becomes significant as the size of that material approaches the nanoscale. Thus in our final example we describe recent work on studying the structure and aggregation of nanoparticles of two minerals.

\section{Molecular Dynamics at the Stable Low Index $\{111\}$ Surface of Ceria}


Materials based on ceria $\left(\mathrm{CeO}_{2}\right)$ are used in the production and purification of hydrogen, the purification of exhaust gases in three-way automotive catalytic converters, and other catalytic applications [2-5]. These applications make use of the unusual properties of ceria, namely, the ability to shift between its two oxidation states, $\mathrm{Ce}$ (III) and $\mathrm{Ce}$ (IV), and the high mobility of bulk oxygen species - properties that allow ceria to behave as an oxygen buffer. It is important therefore that we understand more fully how oxygen atoms move in the ceria surface,. In this study, we focused on studying transport at or near the $\{111\}$ surface of ceria, chosen because it is the most stable surface [8] and hence most prevalent.

The METADISE code (minimum energy techniques applied to dislocation, interface, and surface energies) [6] was used to construct the surface slabs for DL_POLY.

A slab of the $\{111\}$ surface was constructed to a thickness of $20.6 \AA$, which is sufficiently thick to allow realistic simulation of bulk ceria at the centre of the slab. The slab contains 996 atoms. $332 \mathrm{Ce}, 664$ O. We chose to use 3D periodicity for computational ease and hence introduced a $50 \AA$ vacuum gap. The Coulombic sums were calculated using Ewald summation precision of $10^{-5}$, which provides a straightforward way of specifying the number of reciprocal lattice vectors and indicates the precision to which the reciprocal component of the Ewald sum is converged.

The potential model used in this work was derived by Balducci et al (1997) [7] and is a modification of a potential derived by Sayle T.X.T. (1994) [8]. We included the shell model of Dick and Overhauser [9] to simulate ionic polarizability of the oxygen ions. In this model, the oxygen ion is represented as a core plus a shell coupled by a harmonic 
spring. The total charge is separated between the shell and the core. In the molecular dynamics simulations the shells were given a mass of $0.5 \mathrm{au}$.

In order to investigate oxygen atom mobility at the stable $\{111\}$ ceria surface a potential of mean force (PMF) method modification to DL_POLY [10] has been used to simulate the pathway and variation in free energy of a single oxygen atom migrating in bulk ceria and also at surface.

The constrained atom is moved towards a vacancy at a constant velocity. The velocity chosen was $100 \mathrm{~m} / \mathrm{s}$, which is fast enough to obtain good statistics in sufficient cpu time but slow enough to allow the neighbouring ions to relax while the atom is migrating. Slower velocities did not make a significant difference to the energy profile.

$$
\Delta A(z)=A(z)-A\left(z_{0}\right)=\int_{z_{0}}^{z} f_{z} d z
$$

$f_{\mathrm{z}}$ : average force in direction of vacancy.

The constrained atom is given a target vector, which is the co-ordinates of the lattice vacancy position. However, the constrained atom is free to move perpendicular to the target vector. The average force of the constrained atom along the target vector is recorded at the end of each time-step. Integration of the force with respect to distance yields the free energy, shown in equation (1), $\Delta A(z)$ is the free energy change of migration, $A(z)$ is the free energy at position $z$ and $A\left(z_{0}\right)$ is the free energy at the initial position. 
Thus the free energy of atom migration can be calculated at various temperatures. Additionally, MD simulation can be run just above zero Kelvin to enable the identification of the pathway and energy change neglecting temperature. This allows for simple (or an initial) analysis of the migration process, without involving complications of nearby atoms excessively hindering the constrained atom during its trajectory. In doing this, entropy effects are ignored, and therefore the energy of migration rather than the free energy of migration, is in fact calculated.

Migration of a single oxygen atom was investigated at the centre of the slab, which represents bulk ceria; just beneath the surface, with an oxygen migrating between bulk and a vacancy position just under the surface; and at the surface, where oxygen migration is simulated between the layer of oxygen atoms just beneath the surface and the surface layer, and also the migration from a surface position to a near surface vacancy.

\section{Oxygen Migration}

The first PMF simulations were carried out on the diffusion of oxygen in the bulk of the slab. We initially assumed that a vacancy was formed without a localised charge compensation, which we envisage as modelling the transport of the unbound oxygen vacancy. The slab was initially run at $300 \mathrm{~K}$ using the NVT ensemble, and the migration path plotted. The results show that the pathway between oxygen sites is linear (see Figure 1) and that the energy of migration is $0.66 \mathrm{eV}$ with the transition state half way between the oxygen sites, see Figure 2. The activation energy agrees very well with the work of Balducci et al.(1997) [11] who calculated $0.65 \mathrm{eV}$ using a series of static energy calculations. 
We can check that the location of the transition state is exactly halfway between the sites by plotting the average force of the constrained atom in the direction of travel in Figure 3. The force passes through zero at $1.05 \AA$ along a total distance of $2.07 \AA$.

We next modelled the trajectory for an oxygen atom migrating from the oxygen layer just below the surface to a vacant site on the surface.

Figure 4 shows the pathway. The first point to notice is the trajectory is now curved.

The energy plot in Figures 5 shows also a marked asymmetry. The transition state in this case is very close to the surface $\sim 0.5 \AA$. Furthermore, Figure 5 shows that the activation energy for the oxygen migration from the surface to the level just beneath is considerably smaller ie. $0.06 \mathrm{eV}$. Interestingly, a greater activation energy, $0.3 \mathrm{eV}$, is required to activate the return of a subsurface oxygen directly to the $\{111\}$ surface from this layer beneath, which is still less than $0.66 \mathrm{eV}$, the bulk value. It also clearly demonstrates that the vacancy is more stable just below the surface.

The next stage was to examine the transport from the surface layers to the bulk. Figure 6 suggests the barrier is $0.67 \mathrm{eV}$, with the sub-surface site $0.2 \mathrm{eV}$ less stable.

Analysis of the trajectory shows that the migration path is not linear as in the bulk simulation, and that the position of the transition state is predicted to be slightly closer to the bulk layer than the sub-surface layer.

We also considered the migration of an oxygen atom across the top of the surface and surprisingly found that the activation energy was $2.5 \mathrm{eV}$. Thus Figure $7 \mathrm{a}$ and $7 \mathrm{~b}$ illustrates how a migrating oxygen atom would move down a layer just under the surface, and then would return to the surface layer leaving a vacancy in the sub-surface layer, 
which is the most stable site for an isolated vacancy, rather than transport across the surface, not unlike the recently proposed mechanism for surface transport by Allan and Harding [12]

We have seen more complex behaviour in some of the simulations, but still involving oxygen movement between the top two layers. Figure 8 shows the initial configuration of a concerted mechanism involving an oxygen atom in the surface layer constrained to move down into a vacancy position just under the surface. The initial constraint is shown by the largest green arrow in order. The constrained migration results in the formation of a vacancy in a less stable position on the surface because vacancies are more stable at a position just under the surface. The result is that, as the constrained oxygen leaves its lattice site to form a vacancy we find that the next oxygen atom fills the empty site and because the energy barriers are so low the next few oxygen atoms move in unison. The net result is that a sub-surface vacancy is formed but can be potentially some distance from the original vacancy.

The concerted motion does however highlight one problem, namely that the movement of the ions in the concerted mechanism causes a resistance to the movement of the constrained atom, thus the resulting free-energy change is the sum of the activation free energies of migration and the free energy for overcoming the drag-force. Thus care must be taken in interpreting the activation free energy.

Another important issue is the effect of charge compensation particularly when the charge compensating defects are bound to the oxygen vacancies. We consider them in the following section. 
We treated the charge compensating defects as localised electrons on neighbouring cerium ions. Thus we first considered (Figure 9a) the oxygen atom migrating directly into a vacant site, which is adjacent to two $\mathrm{Ce}^{3+}$ ions. The trajectory of migration takes the oxygen between the two adjacent $\mathrm{Ce}^{3+}$ ions. The effect of the charge compensating $\mathrm{Ce}^{3+}$ ions on the migration is significant. The new activation energy is $1.35 \mathrm{eV}$ (Figure 10), compared to $0.06 \mathrm{eV}$ for similar migration without the involvement of compensating ions.

Another interesting result of this calculation is that the final configuration, with the vacancy just below the surface is calculated to be $0.4 \mathrm{eV}$ less stable. But note that the final oxygen vacancy position is not in the highly bound state (i.e.) just under the surface and adjacent to the charge compensating $\mathrm{Ce}^{3+}$ ions.

The second example (Figure 11a) considered is where an oxygen atom just beneath the surface is forced to migrate to a surface site that is adjacent to both, the vacancy and a compensating $\mathrm{Ce}^{3+}$ ion, (i.e.) the oxygen vacancy moves from the top layer to the layer underneath. In this case, the activation energy of migration (Figure 12) is similar to that for the un-charged compensated migration, (ie) $0.10 \mathrm{eV}$ cf. $0.06 \mathrm{eV}$.

The activation energy for the reverse process is approximately $1.0 \mathrm{eV}$, thus in common with the un-charged compensated migration, the vacancy is more stable below the surface but is very much more stabilised i.e., by about $0.9 \mathrm{eV}$.

Hence the energetics for the migration of an oxygen atom into a bound charge compensated vacancy cluster, when that oxygen atom approaches the vacancy from the 
side of the compensation, differ significantly from the case of migration between the compensating ions.

The final example considered (Figure 13a) involves the migration of an oxygen atom, which is originally under the surface between two adjacent $\mathrm{Ce}^{3+}$ ions, to a vacancy position set just to the side of this charge compensating pair of ions. The direction of migration is away from the charge compensation up into the vacancy which is considered here only to be partially bound because the vacancy is not symmetrically configured. Thus the final configuration leaves the vacancy in the highly bound state.

The plot in Figure 14 shows that the activation energies are still small, at $0.25 \mathrm{eV}$ for the forward and $0.4 \mathrm{eV}$ for the reverse.

However, activation migration energy is 4 times greater than the energy for the uncharged compensated case. It is 2.5 times greater than the migration into a bound charge compensated vacancy with a trajectory from the side, but is much smaller than the $1.35 \mathrm{eV}$ required for activation of migration up and between charge compensating ions into the fully bound vacancy.

The results show that energetically the vacancy prefers to be bound to the charge compensating defects in the subsurface oxygen site. Furthermore, the location of these charge compensating defects modify the activation energies and hence the mobility of oxygen near the surface. In the case of the movement along the site of the $\mathrm{Ce}^{3+}$ ions, the effect is significant and will have a large anisotropic effect on the atom transport.

The results therefore suggest that the migration of a defect cluster differs from isolated defects. Therefore, it is important to investigate the stability of different clusters. 
These simulation results suggest an important significance in the direction of migration around or between partially or fully bound charge compensated defects, in that it appears that oxygen atoms prefer to migrate up from the side of a bound compensated surface vacancy.

Lastly, it is important to note that a number of ab initio studies, for example Watson et al.[13-16], have been performed on ceria. Comparison between the ab initio and potential based approaches used here, see for example [17], have shown that the potentials are reliable.

\section{Organic molecules adsorbed on clay surface: DL_POLY as a high-throughput engine}

Organic pollutants and related molecules can pose a serious environmental threat to the global ecosystem. Amongst the most notorious are halogenated compounds substances used as pesticides or as by-products from other human activities. There are concerns because of their general toxicity and the fact they can enter the food chain, as well as affecting the quality of water, soil and atmosphere. In the absence of well thought remediation plans, risk management strategies can give information needed to allow 
stakeholders to decide options concerning disposal of these potentially harmful chemicals.

There have been numerous investigations involving the modelling of clay surfaces and adsorption of organic species [18-21]. However, our interest lies in the understanding of how these molecules might bind to mineral surfaces and in establishing a high-throughput methodology that can efficiently investigate the many possible combinations of molecules with substrates.

As a starting point, we choose the polychloro-dibenzo-pdioxins (PCDDs) family. The 76 members of this family (namely, congeners) have a different number of chlorine atoms replacing hydrogen atoms at different positions.

We then choose a clay substrate, of relevence to soils.. The di-octahedral 2:1 sheet silicate pyrophillite, $\mathrm{Al}_{4} \mathrm{Si}_{8} \mathrm{O}_{20}(\mathrm{OH})_{4}$. is a simple example of the family of clay minerals that form a significant fraction of many soil and rock types. Its [001] face was first considered.

Preliminary DFT calculations showed that the organic molecules do not chemisorb on the pyrophillite surface. Of course, DFT is not adapted to the accurate study of physisorption phenomena due to its poor treatment of dispersion. Therefore our potential based method was deemed ideal for such a study.

We now describe the choices of models, tools and resources, and then follow with a brief discussion of the automatisation procedures and comments on the scientific results.

\section{Model}

A classical model requires an electrostatic description of atoms as well as a parameterisation of the intra-molecular and the inter-molecular interactions. 
Well established models for Zeolite and clay minerals make modeling the substrate a comparatively straightforward task. We chose the parameterisation of Lewis and Catlow [22] in which the electrostatics is described by the shell model of Dick and Overhauser [9]. The total charge is separated between the shell and the core. In the molecular dynamics simulations the shells were given a mass of $0.5 \mathrm{au}$.

For the molecules, we chose the CVFF model [23] for the bonding interactions. The attribution of the electrostatic components was more complex. We used ab initio calculations (Gaussian code [24]) with the Dunning/Huzinaga valence double-zeta basis set on selected PCDDs'. From these we extracted the charge distribution to extrapolate point charges for all the PCDD. This simplified charge model is described in table 1.

\begin{tabular}{lc}
\hline \hline \multicolumn{1}{c}{ Specie } & Charge $(\mathrm{e})$ \\
\hline $\mathrm{H}$ & .20 \\
$\mathrm{Cl}$ & -.03 \\
$\mathrm{O}$ & -.50 \\
$\mathrm{C}(\mathrm{H}$ bonded $)$ & -.20 \\
$\mathrm{C}(\mathrm{Cl}$ bonded $)$ & .03 \\
$\mathrm{C}(\mathrm{O}$ bonded $)$ & .25 \\
\hline \hline
\end{tabular}

Table 1: Charge model (in e)

We generated the interaction parameters between the substrate and the adsorbed molecules by using the standard combination rules, which is a valid approach to obtain dispersion-repulsion interaction coefficients in the absence of strong bonding.

Our confidence in this model is confirmed by the excellent agreement of test calculations on the adsorption of simpler molecules (linear and cyclic aliphatic hydrocarbons) with 
experimental measurements $[18,25]$ : in the case of cyclohexane, $-57 \mathrm{~kJ} \cdot \mathrm{mol}^{-1}$ compared to $-54 \mathrm{~kJ} \cdot \mathrm{mol}^{-1}$ at $423 \mathrm{~K}$.

\section{DL_POLY as an optimiser}

DL_POLY is the UK's national flagship package for molecular dynamics (MD) simulations [26]. While it is especially good for simulating very large systems, its modular structure makes it very adaptable. It supports some form of energy optimisation (albeit not as efficiently as specialist codes such as GULP [27] or METADISE [6]). It is also $\mathrm{cml}$ enabled which allows for certain direct inter-operability with some DFT codes.

The choice of a MD code for a problem essentially based on optimisation might appear to be odd at first. However, our main objective is to tackle adsorption in realistic conditions, i.e. at various temperatures and with the involvement of water. Molecular Dynamics is the tool of choice for these more advanced problems. So, to maintain upward compatibility, we decided to use the same code for the current study as we would for the more advanced one, even though there is added complexity using a MD code to perform optimisation

Additionally, the choice was justified because the force field involved was designed with the aspects of the larger study in mind and is therefore relatively complex and not adaptable to GULP or METADISE codes without heavy recoding .

\section{UCL Condor Pool}

This project is combinatorial in nature. That is, not only are there seventy-six non equivalent PCDD molecules, but the determination of reliable adsorption sites requires multiple starting configurations. Furthermore, the search space is large and the potential 
energy surface can be complex and thus contains many local minima. However, the systems are not overly large and each energy calculation is not too computationally demanding. A brute force approach to optimisation is therefore deemed appropriate and indeed, affordable.

Instead of using a supercomputer, we took advantage of the UCL Condor pool. This type of resource is ideal for this study, as it contains a very large amount of processors (at the time of writing 960 CPUs, operating under Windows OS.

Another feature of the Condor setup is the DAGMAN workflow manager which allows complex workflows. In our case, it essentially made the analysis task automatic.

\section{Methodology}

First of all, three sets of independent calculations are required: molecule only (M), substrate only $(\mathrm{S})$, molecule adsorbed on substrate $(\mathrm{M}+\mathrm{S})$. The adsorption energy is

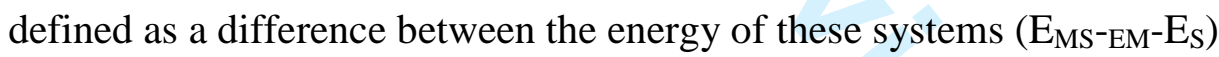

The two custom files node_list and atom_list are at the centre of the data structure and operate on the two modified DL_POLY input files FIELD and CONFIG. node_list contains seventy six lines, one for each PCCD, starting with a user provided molecule code, and then twenty two intermediate atom codes, in the order in which the atoms appear in the CONFIG file (these codes can be $\mathrm{H}, \mathrm{Cl}, \mathrm{O}, \mathrm{C}, \mathrm{CH}, \mathrm{CCl}$ or $\mathrm{CO}$ ). atom_list creates a link between these intermediary codes and the names used in the CONFIG $(H$, $\mathrm{Cl}, \mathrm{O}, \mathrm{C}$ ), as well as with the charges appearing in the FIELD file (as the carbon atoms can be allocated different charges). It also contains information concerning the bonding parameters, which need to be allocated in the FIELD file. 
A list of dedicated scripts reads the node_list and operates on the 76 corresponding directories.

The simpler scripts are just expansions of unix commands (list_cp, list_mv, list_grep). list_setdir generates the data structure (directories NODE_n-moleculecode, where $\mathrm{n}$ is an arbitrary molecule index, corresponding to the order in node_list). list_config replaces <list> flags in a modified CONFIG by the correct atom name and put the correct CONFIG in its directory. list_field replaces <list> and < harm> flags in the field by their value from the atom_list (atom name and charge, two specie bonds parameters). list_fire submit the jobs to the condor scheduler (via run.dlp files). list_rota, is an expanded version of list_config which additionally applies a rotation and/or translation to the flagged atoms of CONFIG (in our case of course those of the PCDD molecule).

These scripts are sufficient to optimise the structures of the isolated molecules.

As already mentioned, the case of the adsorbed molecules is slightly more complex as several starting configuration are needed. Each of these is carried sequentially, for each molecule. The script config_rota, a same directory, command line version of list_rota is used in conjunction with DAGMAN. list_fire is also updated to list_fire_dag. Practically, three sites of high symmetry were explored (above $\mathrm{Si}$, above O, above "hexagon" centre, see figure 16) with 8 rotational configuration (by increment of pi/2), with 24 calculations per molecule.

\section{Results and discussion}

In Figure 15 we plot the adsorption energy of the 76 PCDDs. The molecule index is only meaningful in so far as it increases with the number of chlorine atoms. The horizontal lines are a guide for the eye and correspond to molecules with the same number of 
chlorine. It can be seen that the adsorption energy is strongly correlated to the number of chlorine. Each additional chlorine attached to the carbon rings leads on average to an adsorption energy increase of $0.03 \mathrm{eV}$.

Furthermore, and as might have been expected, all of the molecules lie flat on the surface, see Figure 16.

We also observe that corrugation energy increases with the number of chlorine. The potential energy surface is very flat for the PCDD with a low $\mathrm{Cl}$ count (indeed the translational corrugation energy is $0 \pm 0.01 \mathrm{eV}$ for $\mathrm{C}_{12} \mathrm{O}_{2} \mathrm{H}_{8}$ ). At higher $\mathrm{Cl}$ count, the corrugation increases, up to $0.07 \mathrm{eV}$ for $\mathrm{C}_{12} \mathrm{O}_{2} \mathrm{Cl}_{8}$. Therefore, the concept of the adsorption site is rather meaningless for molecules with numbers of $\mathrm{Cl}$ lower than 4 . For molecules with greater than $3 \mathrm{Cl}$ atoms, the PCDD almost systematically favours a position such as illustrated in Figure 16, with the centre of mass of the molecule situated above a surface oxygen, and where the benzene rings sit inside the quasi-hexagon of the surface top layer.

As well as the modelling of molecules that are in contact with surfaces, we are also concerned with the interaction of nanoparticles.

\section{Molecular dynamics simulations of the structural changes that occur during aggregation of mineral oxide and carbonate nanoparticles}

DL_POLY has been used by other groups to study the structure and stability on minerals on the nano-scale. Spano et al found computational evidence of $\mathrm{ZnS}$ forming bubble type 
clusters [28] $(\mathrm{ZnS})_{\mathrm{n}}$ clusters, with $\mathrm{n}$ ranging from 10 to 47 , were energy minimised using a simulated annealing technique. Spano et al found that spheroidal (bubble) clusters with only three coordinated atoms are more stable than the bulk structure. If the number of $\mathrm{ZnS}$ units is increased to 60 then the appearance of onion structures occur [29]. This is a $(\mathrm{ZnS})_{12}$ inside a bubble of $(\mathrm{ZnS})_{48}$. Banfield and co workers have also considered $\mathrm{ZnS}$ nanoparticles, typically $\mathrm{n}=360-400$, making the diameter of the $\mathrm{ZnS}$ crystal around $3 \mathrm{~nm}$, which is comparable to experimental synthesised particles [30]. The Banfield group started using molecular dynamics to study the phase stability of $\mathrm{ZnS}$ nanoparticles in vacuum and in the presence of water. They found that the wurtzite $(\mathrm{ZnS})$ nanoparticles smaller than $7 \mathrm{~nm}$ are more stable than sphalerite nanoparticles in vacuum at $300 \mathrm{~K}$. However adsorption of water onto the $\mathrm{ZnS}$ nanoparticle surface stabilizes the sphalerite structure. Zhang et al [31] predicted anhydrous $\mathrm{ZnS}$ nanoparticles to have a highly disordered structure. Again these nanoparticles had a diameter of around $3 \mathrm{~nm}$. The adsorption of water, however, onto the nanoparticle surface results in the formation of a relatively crystalline core. Zhang and Banfield showed that this phase transformation of sphalerite to wurtzite could be caused by nanoparticle aggregation and coarsening [32]. These $3 \mathrm{~nm} \mathrm{ZnS}$ nanoparticles were randomly orientated with respect to each other and allowed to aggregate to form a larger nanoparticle. Following aggregation the coarsened particle adopts a near-amorphous structure that transforms rapidly to wurtzite. This work by Banfield and co-workers has provided a link between atomistic simulation and the forces that cause their aggregation, i.e. colloidal chemistry. We have used DL_POLY to extend the range of materials to simulate the aggregation process of two different materials, $\mathrm{MgO}$ and calcite $\left(\mathrm{CaCO}_{3}\right)$ nanoparticles. First we will describe the potentials 
used to describe the interatomic interactions and then describe how we set up our systems for aggregation.

The potential model used for simulating the magnesium-oxygen interaction is that of Lewis and Catlow [33] and has been used before to simulate $\mathrm{MgO}$ surfaces [34, 35] and $\mathrm{MgO}$ nanocrystals [36]. The potential model used for simulating the calcium-carbonate interactions in calcite is that of Pavese et al [37] and has been used in the past to simulate calcite surfaces [38] and also calcite nanoparticles [39]. All simulations in this section employ the shell model of Dick and Overhauser [9]. Where the shells are in the oxygen species only and have a mass of 0.2 au in $\mathrm{CaCO}_{3}$ and 0.5 in $\mathrm{MgO}$, this represents the electronic charge cloud connected by a harmonic spring to a core containing most of the anion's mass.

We have begun studying the structure and aggregation using molecular dynamics simulations by modelling an eight nanoparticle system. Each of the nanoparticles in the simulation is the same distance apart. The simulation was performed in vacuum with no periodic boundary conditions. The molecular dynamic simulation was performed in a canonical ensemble at $300 \mathrm{~K}$ and ambient pressure using a time step of $0.1 \mathrm{fs}$. Each simulation was run for $1 \mathrm{~ns}$ with a 2 ps equilibration period.

\section{Aggregation of Magnesium Oxide nanoparticles}

Each $\mathrm{MgO}$ nanoparticle had a $0.6 \mathrm{~nm}$ in diameter and the simulation started with them being $2.3 \mathrm{~nm}$ apart. The starting configuration is shown in figure 17 . We have 8 cubes of $\mathrm{MgO}$ as the most stable surface grows along the $\{100\}$ plane [6]. We can plot the potential energy of our simulation as it evolves over time (figure 18). The starting 
configuration has a potential energy of $-482.1 \mathrm{~kJ} / \mathrm{mol}$ of $\mathrm{Mg}$ ions and then drops rapidly in-between $30-55 \mathrm{ps}$ to $-484.4 \mathrm{~kJ} / \mathrm{mol}$. This decrease in potential energy corresponds to the initial aggregation of $\mathrm{MgO}$ nanoparticles. Figure 19 shows a snapshot of the MD simulation at 30ps. The particles retain their bulk-like structure and well ordered surfaces. At 30ps single $\mathrm{MgO}$ nanoparticles are starting to aggregate together in pairs. The aggregation process seems very ordered and is dominated by orientation along the $\{100\}$ plane. This snapshot is also interesting because it also shows that the merging nanoparticles will join at the corners first. The pair of nanoparticles that are circled in figure 19 are a pair that drifts further and further away from the rest of the nanoparticles and don't aggregate with the other six nanoparticles. Since they are so far away in some of the snapshots it is not practical to show them in some of the pictures. At 60ps figure 20 shows a stabilisation of potential energy at around $-484.4 \mathrm{~kJ} / \mathrm{mol}$. Figure 20 shows a snapshot of the MD simulation at 60ps and shows the aggregation of a single $\mathrm{MgO}$ nanoparticle to a pair. The snapshot again illustrates the general result that aggregation has initially occurred along the corners of the nanoparticles. The nanoparticles find their preferred face centre cubic site at 220ps (figure 21) when the potential decreases to around $-484.6 \mathrm{~kJ} / \mathrm{mol}$. The increased ordering of aggregation along the $\{100\}$ plan continues when the potential energy decreases to $-485.8 \mathrm{~kJ} / \mathrm{mol}$ when a pair of nanoparticles aggregate to the triplet at $275 \mathrm{ps}$ (figure 22). The final configuration occurs at 460ps (figure 23). Here the sixth nanoparticle joins the cluster of five. The two nanoparticles circled in Figure 19 were in fact too far away to become attracted to the rest of them. This aggregation process has also shown that when crystal growth occurs in $\mathrm{MgO}$ there are the chances of kinks and steps being formed. 
We can compare any structural changes that may have occurred during aggregation by comparing the radial distribution functions (RDF) of aggregated nanoparticles with bulk $\mathrm{MgO}$. Figure 24 shows the RDF of the (a) aggregated $\mathrm{MgO}$ nanoparticle and (b) bulk $\mathrm{MgO}$. The RDF's of the aggregated $\mathrm{MgO}$ nanoparticle and bulk $\mathrm{MgO}$ are very similar with the first three peaks showing $\mathrm{Mg}-\mathrm{O}$ bond distances at $0.21,0.35$ and $0.45 \mathrm{~nm}$ in both graphs. These interatomic distances agree with x-ray diffraction experiments $[40,41]$. The RDF of the aggregated nanoparticle shows that during aggregation the $\mathrm{MgO}$ nanoparticles continue to maintain their crystalline form. Furthermore, the bonding between the $\mathrm{MgO}$ is so highly directed, resulting in a continuous structure. Thus although the overall shape is non-symmetric there are no obvious grain boundaries being formed. Next we show the aggregation process of eight calcite nanoparticles.

\section{Aggregation of calcite nanoparticles}

Eight calcite nanoparticles that are $1.6 \mathrm{~nm}$ in diameter are placed $1.7 \mathrm{~nm}$ apart. The starting configuration is shown in figure 25 . The simulation was run for 1 ns. Figure 28 shows the potential energy of the system as a function of MD time. The simulation starts with a potential energy of $-715.3 \mathrm{~kJ} / \mathrm{mol}$ of calcium ions. As the simulation evolves the first drop in potential energy occurs at around 50ps. This corresponds to figure 27 which shows that before aggregation occurs the individual nanoparticles will relax on their own to a potential energy of $-715.6 \mathrm{~kJ} / \mathrm{mol}$, which corresponds to a more disordered structure. Once the calcite particles are relaxed aggregation occurs in pairs. At around 67ps the eight nanoparticles have formed four nanoparticles and the energy lowers to $716.9 \mathrm{~kJ} / \mathrm{mol}$. Figure 28 shows a snapshot of aggregation at $87 \mathrm{ps}$. The potential energy of 
this system continues to lower, $-718.2 \mathrm{~kJ} / \mathrm{mol}$, as the four calcite nanoparticles start to form two larger nanocrystals. The two larger calcite nanoparticles now become attracted to each other and again as they merge the potential energy drops to $-719.9 \mathrm{~kJ} / \mathrm{mol}$ at around 140ps (figure 29. The final configuration occurs at 180ps and the potential energy stabilises at $-721.0 \mathrm{~kJ} / \mathrm{mol}$. Figure 30 shows the aggregated nanoparticle, which has lost its symmetry. The final nanoparticle has a longest diameter of $4.17 \mathrm{~nm}$ and a width of $1.816 \mathrm{~nm}$. Figure 31 a shows the RDF of the final aggregated calcite nanoparticle. When compared with the RDF of bulk calcite (Figure 31b) it has much less order and is more amorphous then the crystalline bulk. The peaks in the RDF bulk calcite shows the same Ca-O bond distances, $0.23,0.33,0.41 \mathrm{~nm}$, as found experimentally $[42,43]$. The average coordination number for a calcium ion in the aggregated nanoparticle is calculated to be around 6.5, which is slightly higher then the coordination number for bulk calcite which is 6. This suggests that the amorphous nanoparticle is more closely related to calcite, rather than aragonite which has a calcium ion coordination number of 8 .

The difference between an aggregated calcite and an aggregated $\mathrm{MgO}$ is that $\mathrm{MgO}$ keeps its bulk-like structure and still has crystalline characteristics. The RDF shows sharp peaks whereas the RDF of the aggregated calcite is more amorphous with only two peaks. The atoms of the calcite nanoparticle seems to be more disordered and has no structural preference for linking different particles whereas the aggregated $\mathrm{MgO}$ has a very uniform structure.

There has been a great deal of debate in the literature suggesting that biomineralisation occurs from amorphous calcium carbonate [44-47]. We have shown that very small calcium carbonate nanoparticles are more stable when amorphous and also when they 
aggregate together their morphology changes drastically. Therefore this aggregation process could promote the exotic morphologies seen in biomineralistion. In future studies we set out to quantify this aggregation process.

\section{Conclusions}

DL_POLY has great scope for our molecular dynamics simulation experiments. We have been able to calculate both the free energy of nanoparticle aggregation and the activation free energy of individual atom migration. DL_POLY has also been shown to be versatile when used as an energy optimiser and for investigating adsorption of molecules on surfaces.

Application of DL_POLY has allowed us to investigate oxygen atom migration at the stable $\{111\}$ surface of ceria. The oxygen migration is shown to be much more energetically favoured at the $\{111\}$ surface than the bulk when via subsurface oxygen vacancies, and the activation free energy of migration was found to be the same in all directions. When considering the effect of neighbouring charge compensating $\mathrm{Ce}^{3+}$ ions the oxygen was found to be less mobile and no longer isotopic. 
We have determined the adsorption energy and sites of the 76 PCDD congeners on a pyrophilite surface, using DL_POLY. The molecules are weakly bonded and the corrugation is low. The variation in energy with adsorption sites is small. The main factor controlling the adsorption energy is the number of chlorine atoms.

The simplicity of the PCDDs adsorption on this surface means that the process can be modelled very simply and parameterised for use in large scale soil remediation models.

We have investigated the differences between calcite and $\mathrm{MgO}$ nanoparticle aggregation. The difference between an aggregated calcite and an aggregated $\mathrm{MgO}$ is that $\mathrm{MgO}$ keeps its bulk-like structure and aggregates in specific orientations. In contrast, aggregated calcite is more amorphous and does not have any noticeable preferred directionality . The structure of the calcite nanoparticle seems to be more mobile whereas the aggregated $\mathrm{MgO}$ has a very uniform structure.

In summary, DL_POLY allows us to carry-out sophisticated molecular dynamics simulations, intricate computational calculations and gives us scope for further investigatory work. Importantly, these applications of DL_POLY are providing a useful complement to experiments. 


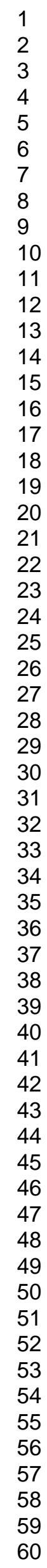

http://mc.manuscriptcentral.com/tandf/jenmol 
References:

1. White, T.O., R.P. Bruin, J. Wakelin, C. Chapman, D. Osborn, P. Murray-Rust, E. Artacho, M.T. Dove, and M. Calleja. eScience methods for the combinatorial chemistry problem of adsorption of pollutant organic molecules on mineral surfaces. Proceedings of the UK e-Science All Hands Meeting 2005. (2005)

2. Deluga, G.A., J.R. Salge, L.D. Schmidt, and X.E. Verykios. Renewable hydrogen from ethanol by autothermal reforming. Science. 303. 5660. (2004)

3. Otsuka, K., T. Ushiyama, and I. Yamanaka. Partial Oxidation of Methane Using the Redox of Cerium Oxide. Chemistry Letters. 9. (1993)

4. Trovarelli, Catalysis by Ceria and Related Materials. 2002, London: Imperial College Press.

5. Park, S.D., J.M. Vohs, and R.J. Gorte. Direct oxidation of hydrocarbons in a solid-oxide fuel cell. Nature. 404. 6775. (2000)

6. Watson, G.W., E.T. Kelsey, N.H. deLeeuw, D.J. Harris, and S.C. Parker. Atomistic simulation of dislocations, surfaces and interfaces in MgO. Journal of the Chemical Society-Faraday Transactions. 92. 3. (1996)

7. Balducci, G., J. Kaspar, P. Fornasiero, M. Graziani, M.S. Islam, and J.D. Gale. Computer simulation studies of bulk reduction and oxygen migration in $\mathrm{CeO} 2-$ ZrO2 solid solutions. Journal of Physical Chemistry B. 101. 10. (1997)

8. Sayle, T.X.T., S.C. Parker, and C.R.A. Catlow. Surface Segregation of Metal-Ions in Cerium Dioxide. Journal of Physical Chemistry. 98. 51. (1994)

9. Dick, A.W. and B.G. Overhauser. Theory of dielectric constants of alkali halide crystals. Physical Reviews. 112. 1. (1958)

10. Kerisit, S. and S.C. Parker. Free energy of adsorption of water and metal ions on the $\{1014\}$ calcite surface. Journal of the American Chemical Society. 126. 32. (2004)

11. Balducci, G., M.S. Islam, J. Kaspar, P. Fornasiero, and M. Graziani. Bulk reduction and oxygen migration in the ceria-based oxides. Chemistry of Materials. 12. 3. (2000)

12. Harris, D.J., M.Y. Lavrentiev, J.H. Harding, N.L. Allan, and J.A. Purton. Novel exchange mechanisms in the surface diffusion of oxides. Journal of PhysicsCondensed Matter. 16. 13. (2004)

13. Nolan, M., S. Grigoleit, D.C. Sayle, S.C. Parker, and G.W. Watson. Density functional theory studies of the structure and electronic structure of pure and defective low index surfaces of ceria. Surface Science. 576. 1-3. (2005)

14. Nolan, M., S.C. Parker, and G.W. Watson. The electronic structure of oxygen vacancy defects at the low index surfaces of ceria. Surface Science. 595. 1-3. (2005)

15. Nolan, M., S.C. Parker, and G.W. Watson. Reduction of NO2 on ceria surfaces. Journal of Physical Chemistry B. 110. 5. (2006)

16. Nolan, M., S.C. Parker, and G.W. Watson. CeO2 catalysed conversion of CO, NO2 and NO from first principles energetics. Physical Chemistry Chemical Physics. 8. 2. (2006) 
17. Parker, S.C., S. Kerisit, A. Marmier, S. Grigoleit, and G.W. Watson. Modelling inorganic solids and their interfaces: A combined approach of atomistic and electronic structure simulation techniques. Faraday Discussions. 124. (2003)

18. Keldsen, G.L., J.B. Nicholas, K.A. Carrado, and R.E. Winans. Molecular Modeling of the Enthalpies of Adsorption of Hydrocarbons on Smectite Clay. Journal of Physical Chemistry. 98. 1. (1994)

19. Skipper, N.T., P.A. Lock, J.O. Titiloye, J. Swenson, Z.A. Mirza, W.S. Howells, and F. Fernandez-Alonso. The structure and dynamics of 2-dimensional fluids in swelling clays. Chemical Geology. 230. 3-4. (2006)

20. Yu, C.H., S.Q. Newton, M.A. Norman, L. Schafer, and D.M. Miller. Molecular dynamics simulations of adsorption of organic compounds at the clay mineral/aqueous solution interface. Structural Chemistry. 14. 2. (2003)

21. Zeng, Q.H., A.B. Yu, G.Q. Lu, and R.K. Standish. Molecular dynamics simulation of organic-inorganic nanocomposites: Layering behavior and interlayer structure of organoclays. Chemistry of Materials. 15. 25. (2003)

22. Lewis, G.V. and C.R.A. Catlow. Potential Models for Ionic Oxides. Journal of Physics C-Solid State Physics. 18. 6. (1985)

23. Dauberosguthorpe, P., V.A. Roberts, D.J. Osguthorpe, J. Wolff, M. Genest, and A.T. Hagler. Structure and Energetics of Ligand-Binding to Proteins Escherichia-Coli Dihydrofolate Reductase Trimethoprim, a Drug-Receptor System. Proteins-Structure Function and Genetics. 4. 1. (1988)

24. Frisch, M.J., G.W. Trucks, H.B. Schlegel, G.E. Scuseria, M.A. Robb, J.R. Cheeseman, J. Montgomery, J. A., T. Vreven, K.N. Kudin, J.C. Burant, J.M. Millam, S.S. Iyengar, J. Tomasi, V. Barone, B. Mennucci, M. Cossi, G. Scalmani, N. Rega, G.A. Petersson, H. Nakatsuji, M. Hada, M. Ehara, K. Toyota, R. Fukuda, J. Hasegawa, M. Ishida, T. Nakajima, Y. Honda, O. Kitao, H. Nakai, M. Klene, X. Li, J.E. Knox, H.P. Hratchian, J.B. Cross, V. Bakken, C. Adamo, J. Jaramillo, R. Gomperts, R.E. Stratmann, O. Yazyev, A.J. Austin, R. Cammi, C. Pomelli, J.W. Ochterski, P.Y. Ayala, K. Morokuma, G.A. Voth, P. Salvador, J.J. Dannenberg, V.G. Zakrzewski, S. Dapprich, A.D. Daniels, M.C. Strain, O. Farkas, D.K. Malick, A.D. Rabuck, K. Raghavachari, J.B. Foresman, J.V. Ortiz, Q. Cui, A.G. Baboul, S. Clifford, J. Cioslowski, B.B. Stefanov, G. Liu, A. Liashenko, P. Piskorz, I. Komaromi, R.L. Martin, D.J. Fox, T. Keith, M.A. AlLaham, C.Y. Peng, A. Nanayakkara, M. Challacombe, P.M.W. Gill, B. Johnson, W. Chen, M.W. Wong, C. Gonzalez, and J.A. Pople, Gaussian 03, Revision C.02. 2004, Gaussian, Inc.: Wallingford CT.

25. Zhukova, A.I., S.V. Bondarenko, and Y.I. Tarasevich. Study of Selectivity of Natural Sorbents with Respect to Hydrocarbons. Ukrainskii Khimicheskii Zhurnal. 42. 7. (1976)

26. Smith, W. and T.R. Forester. DL_POLY_2.0: A general-purpose parallel molecular dynamics simulation package. Journal of Molecular Graphics. 14. 3. (1996)

27. Gale, J.D. and A.L. Rohl. The General Utility Lattice Program (GULP). Molecular Simulation. 29. 5. (2003)

28. Spano, E., S. Hamad, and C.R.A. Catlow. Computational evidence of bubble ZnS clusters. Journal of Physical Chemistry B. 107. 38. (2003) 
29. Spano, E., S. Hamad, and C.R.A. Catlow. ZnS bubble clusters with onion-like structures. Chemical Communications. 7. (2004)

30. Zhang, H.Z., F. Huang, B. Gilbert, and J.F. Banfield. Molecular dynamics simulations, thermodynamic analysis, and experimental study of phase stability of zinc sulfide nanoparticles. Journal of Physical Chemistry B. 107. 47. (2003)

31. Zhang, H.Z., B. Gilbert, F. Huang, and J.F. Banfield. Water-driven structure transformation in nanoparticles at room temperature. Nature. 424. 6952. (2003)

32. Zhang, H.Z. and J.F. Banfield. Aggregation, coarsening, and phase transformation in $\mathrm{ZnS}$ nanoparticles studied by molecular dynamics simulations. Nano Letters. 4. 4. (2004)

33. Lewis, G.V. and C.R.A. Catlow. Potential model for ionic oxides. Journal of Physical Chemistry: Solid State Physics. 18. (1985)

34. de Leeuw, N.H. and S.C. Parker. Molecular-dynamics simulation of MgO surfaces in liquid water using a shell-model potential for water. Physical Review B. 58. 20. (1998)

35. Harris, D.J., J.H. Harding, and S.C. Parker. Simulations of surfaces and interfaces in MgO. Radiation Effects and Defects in Solids. 151. 1-4. (1999)

36. Sayle, D.C., J.A. Doig, S.A. Maicaneanu, and G.W. Watson. Atomistic structure of oxide nanoparticles supported on an oxide substrate. Physical Review B. 65. 24. (2002)

37. Pavese, A., M. Catti, S.C. Parker, and A. Wall. Modelling of the thermal dependence of structural and elastic properties of calcite, $\mathrm{CaCO}$. Physics and Chemistry of Minerals. 23. 2. (1996)

38. Kerisit, S. and S.C. Parker. Free energy of adsorption of water and calcium on the \{10(1)over-bar-4\} calcite surface. Chemical Communications. 1. (2004)

39. Kerisit, S., D.J. Cooke, D. Spagnoli, and S.C. Parker. Molecular dynamics simulations of the interactions between water and inorganic solids. Journal of Materials Chemistry. 15. 14. (2005)

40. Bragg, W.L. Crystal structure. Nature. 105. (1920)

41. Tsirel'son, V.G.A., A.S.;Abramov, Yu.A.;Belokoneva, E.L.;Kitaneh, R.;Feil, D. X-ray and electron diffraction study of Mg O. Acta Crystallographica B. 54. (1998)

42. Maslen, E.N.S.t., V.A.;Strel'tsova, N.R. X-ray study of the electron density in calcite, CaCO3. Acta Crystallographica B. 49. (1993)

43. Wyckoff, R.W.G. The crystal structures of some carbonates of the calcite group. American Journal of Science, Serie 50. (1920)

44. Addadi, L., S. Raz, and S. Weiner. Taking advantage of disorder: Amorphous calcium carbonate and its roles in biomineralization. Advanced Materials. 15. 12. (2003)

45. Faatz, M., F. Grohn, and G. Wegner. Amorphous calcium carbonate: Synthesis and potential intermediate in biomineralization. Advanced Materials. 16. 12. (2004)

46. Weiner, S., Y. Levi-Kalisman, S. Raz, and L. Addadi. Biologically formed amorphous calcium carbonate. Connective Tissue Research. 44. (2003)

47. Xu, X.R., J.T. Han, and K. Cho. Formation of amorphous calcium carbonate thin films and their role in biomineralization. Chemistry of Materials. 16. 9. (2004) 


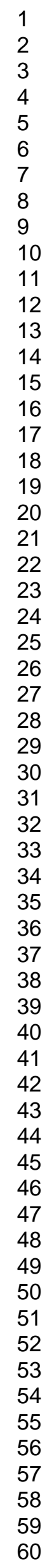

http://mc.manuscriptcentral.com/tandf/jenmol 


\section{Table of Figures}

Figure 1: Oxygen Atom Migrating in The Middle of $\{111\}$ Surface Slab

Figure 2: Energy Profile of Bulk Oxygen Migration

Figure 3: Plot Showing Constrained Force Experienced by the Migrating Atom over Distance Travelled in Bulk Ceria.

Figure 4: Oxygen Atom Migrating in the $\{111\}$ Surface.

Figure 5: Energy Profile of Oxygen Migration in the $\{111\}$ Surface.

Figure 6: Energy Profile of Oxygen Migration from Bulk to Just Under the $\{111\}$ Surface.

Figure 7 (a) Schematic of oxygen hopping across the surface.

Figure 7 (b) Schematic of oxygen moving through the surface.

Figure 8: Pathway of Concerted Mechanism

Figure 9a: Before migration and with Surface Vacancy in place.

Figure 9b: After Oxygen atom, originally under the surface between $\mathrm{Ce}^{3+}$ ions, has migrated to the surface leaving vacancy just under the surface and between $\mathrm{Ce}^{3+}$ ions.

Figure 10: Energy Profile of Oxygen atom migration between $\mathrm{Ce}^{3+}$ ions onto the Surface.

Figure. 11a: Before migration and with Surface Vacancy in place.

Figure. 11b: After. Oxygen atom, originally under the surface and below the vacancy, has migrated to the surface leaving vacancy just under the surface and below the left $\mathrm{Ce}^{3+}$ ion.

Figure 12: Energy Profile of Oxygen atom migration From under the Left Hand $\mathrm{Ce}^{3+}$ ion into the Surface Vacancy between Compensating $\mathrm{Ce}^{3+}$ ions.

Figure 13a: Before migration and with Surface Vacancy in place.

Figure 13b: After. Oxygen atom, originally under the surface between $\mathrm{Ce}^{3+}$ ions, has migrated to the surface leaving vacancy just under the surface and between $\mathrm{Ce}^{3+}$ ions.

Figure 14: Energy Profile of Oxygen atom migration From under the Left Hand $\mathrm{Ce}^{3+}$ ion into the Surface Vacancy between Compensating $\mathrm{Ce}^{3+}$ ions.

Figure 15: Adsorption energy of the 76 PCDD 
Figure 16: Adsorption position of a PCDD congener. The surface is parallel to the page. The shaded atoms represent the lower layers. Surface: Si Large light grey, O small dark grey. Molecule: $\mathrm{H}$ white, $\mathrm{Cl}$ light grey, $\mathrm{C}$ grey, $\mathrm{O}$ dark grey.

Figure 17: Initial configuration of eight $\mathrm{MgO}$ nanoparticles before aggregation.

Figure 18: Potential energy per magnesium ion against time of an MD simulation of 8 $\mathrm{MgO}$ nanoparticles

Figure 19: A snapshot of the MD simulation at 30ps for the $\mathrm{MgO}$ system

Figure 20: A snapshot of the MD simulation at 60ps for the $\mathrm{MgO}$ system

Figure 21: A snapshot of the MD simulation at 220ps for the $\mathrm{MgO}$ system

Figure 22: A snapshot of MD simulation at 275ps for the $\mathrm{MgO}$ system

Figure 23: Final configuration of the aggregated $\mathrm{MgO}$ nanoparticles

Figure 24: RDF of the (a) aggregated $\mathrm{MgO}$ nanoparticles and (b) bulk $\mathrm{MgO}$

Figure 25: Initial configuration of calcite nanoparticles for MD simulation

Figure 26: Potential energy per calcium ion against time of an MD simulation of 8 calcite nanoparticles

Figure 27: A snapshot of the MD simulation at 50ps for the calcite $1.6 \mathrm{~nm}$ system

Figure 28: A snapshot of the MD simulation at $87 \mathrm{ps}$ for the calcite $1.6 \mathrm{~nm}$ system

Figure 29: A snapshot of the MD simulation at 140ps for the calcite 1.6nm system

Figure 30: Final configuration of the aggregated calcite nanoparticles

Figure 31: RDF of the (a) aggregated calcite nanoparticles and (b) bulk calcite 


\section{Page 137 of 158}

1

2

3
4

4

5

6
7

8

9

10

11

12
13

14

15

16

17

18

19

20

21

22

23

24

25

26

27

28

29

30

34

35

36

37

38

39

40

41

42

43

44

45

46

47

48

49

50

51

52

53

54

55

56

57

58

59

60

http://mc.manuscriptcentral.com/tandf/jenmol 


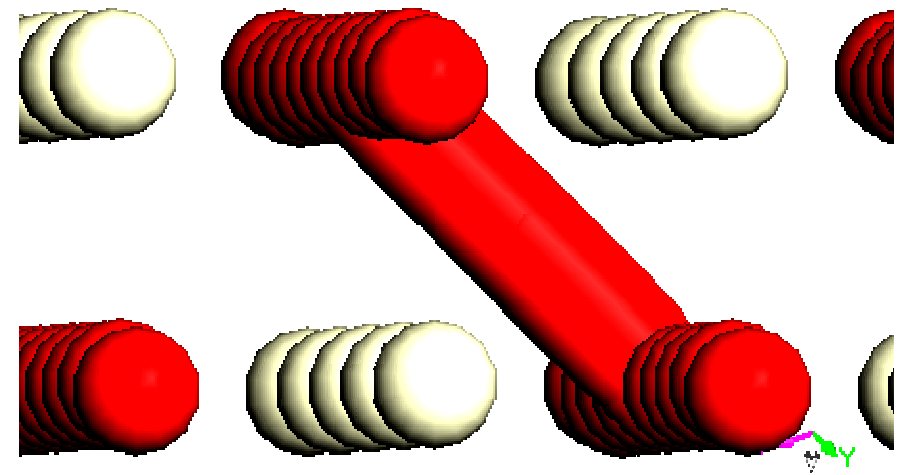

Figure 1: Oxygen Atom Migrating in The Middle of $\{111\}$ Surface Slab

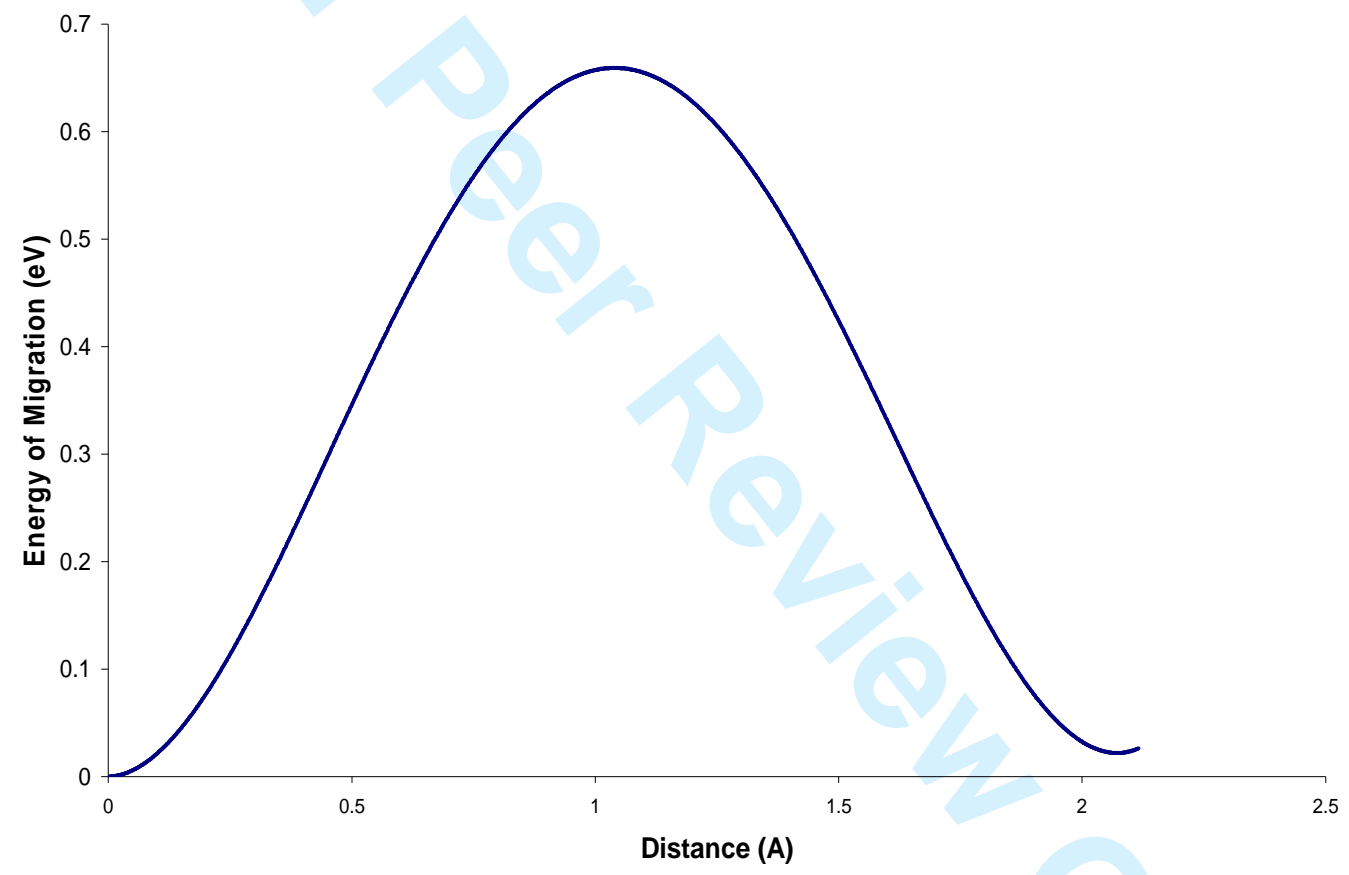

Figure 2: Energy Profile of Bulk Oxygen Migration 


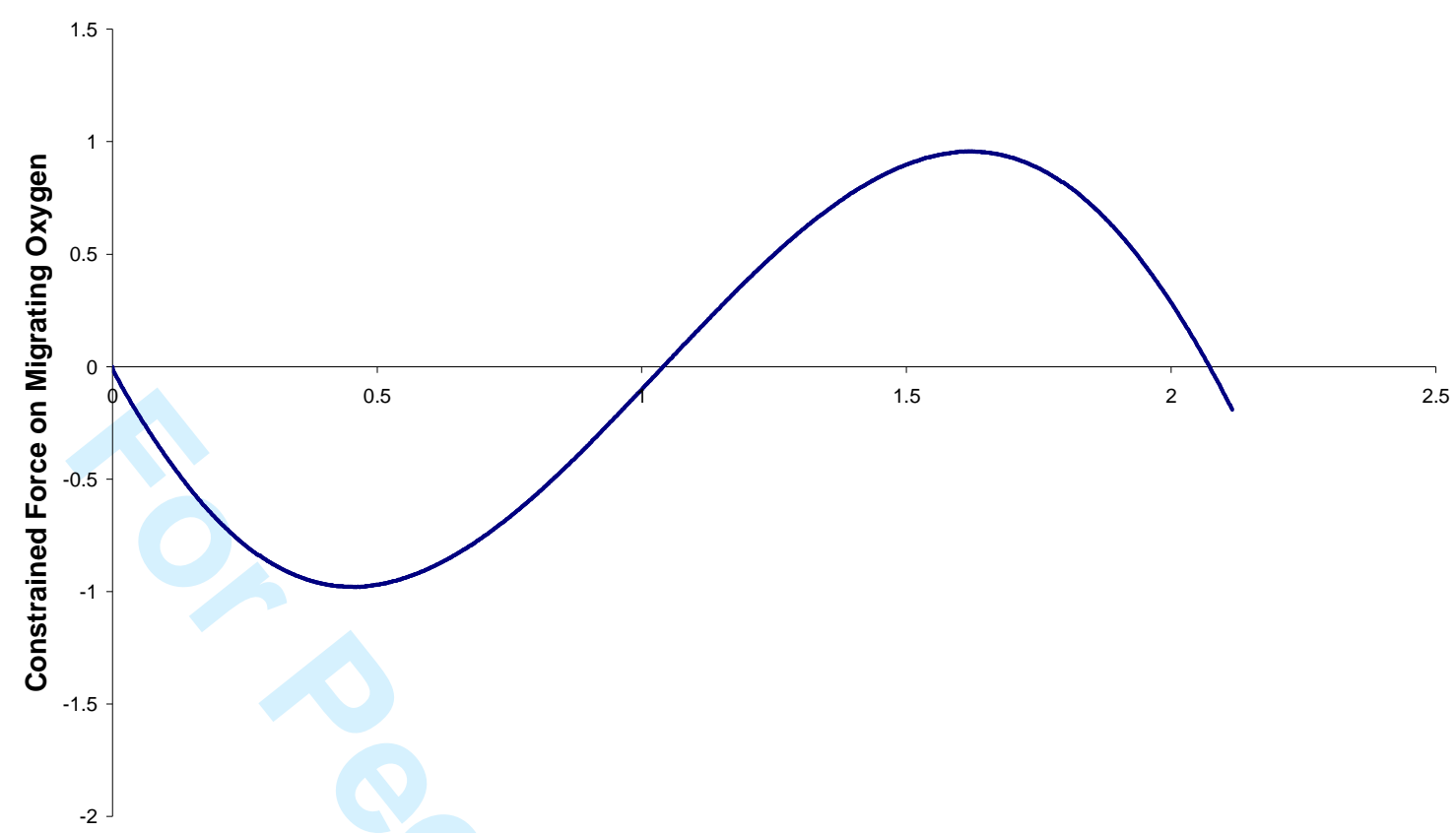

Distance (A)

Figure 3: Plot Showing Constrained Force Experienced by the Migrating Atom (in $\mathrm{eV} / \mathrm{A}$ ) over Distance Travelled in Bulk Ceria

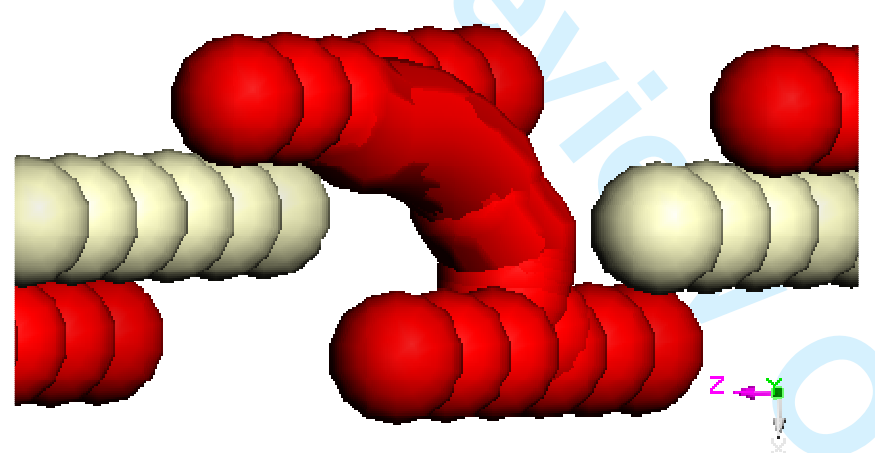

Figure 4: Oxygen Atom Migrating in the $\{111\}$ Surface 


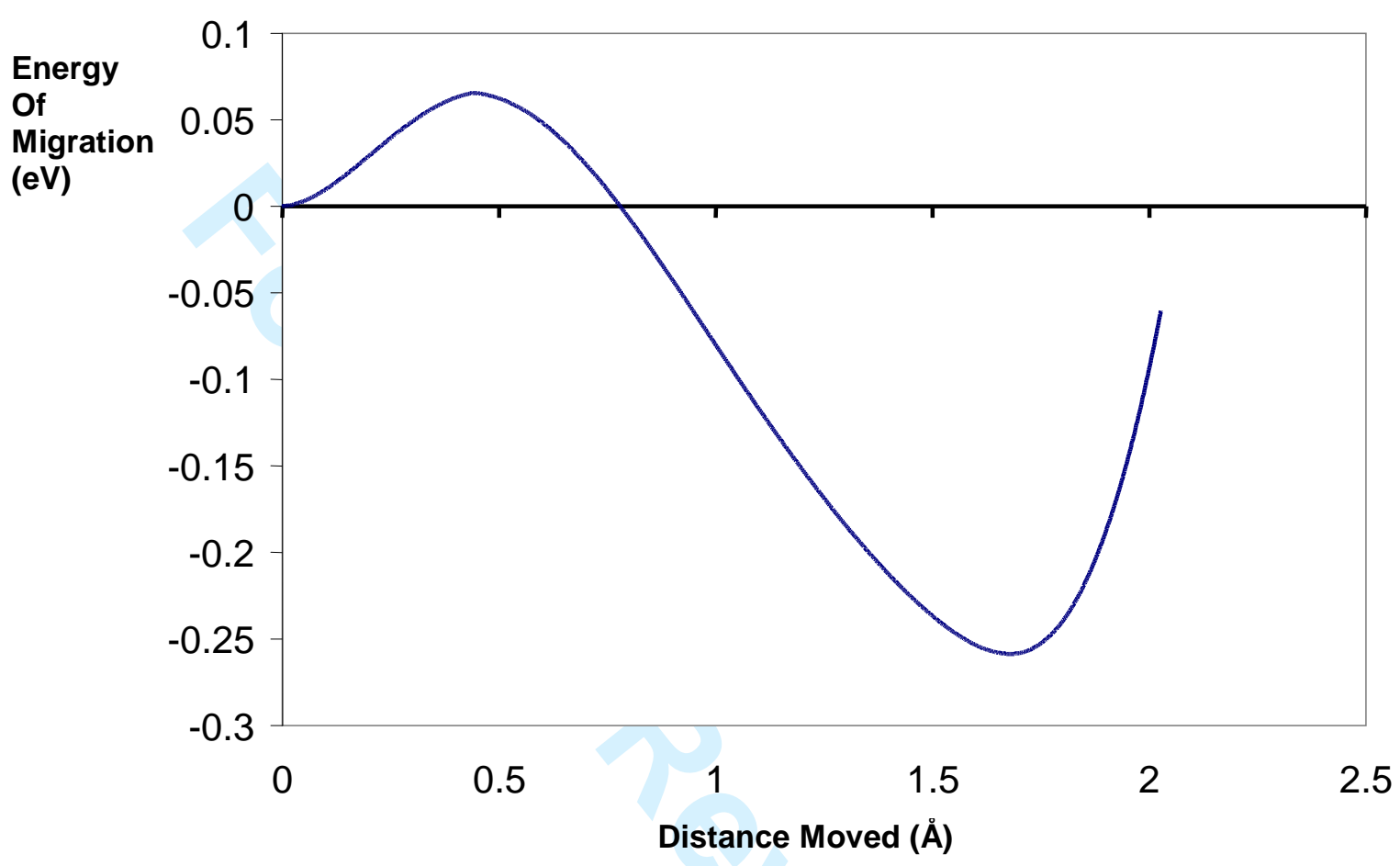

Figure 5: Energy Profile of Oxygen Migration in the $\{111\}$ Surface. 
1

2

3

4

5

6

7

8

10

11

12

14

15

16

17

18

19

20

21

22

23

24

25

26

27

28

29

30

31

32

33

34

35

36

37

38

39

40

41

42

43

44

45

46

47

48

49

50

51

52

53

54

55

56

57

58

59

60

\section{Energy of \\ Migration}

(eV)

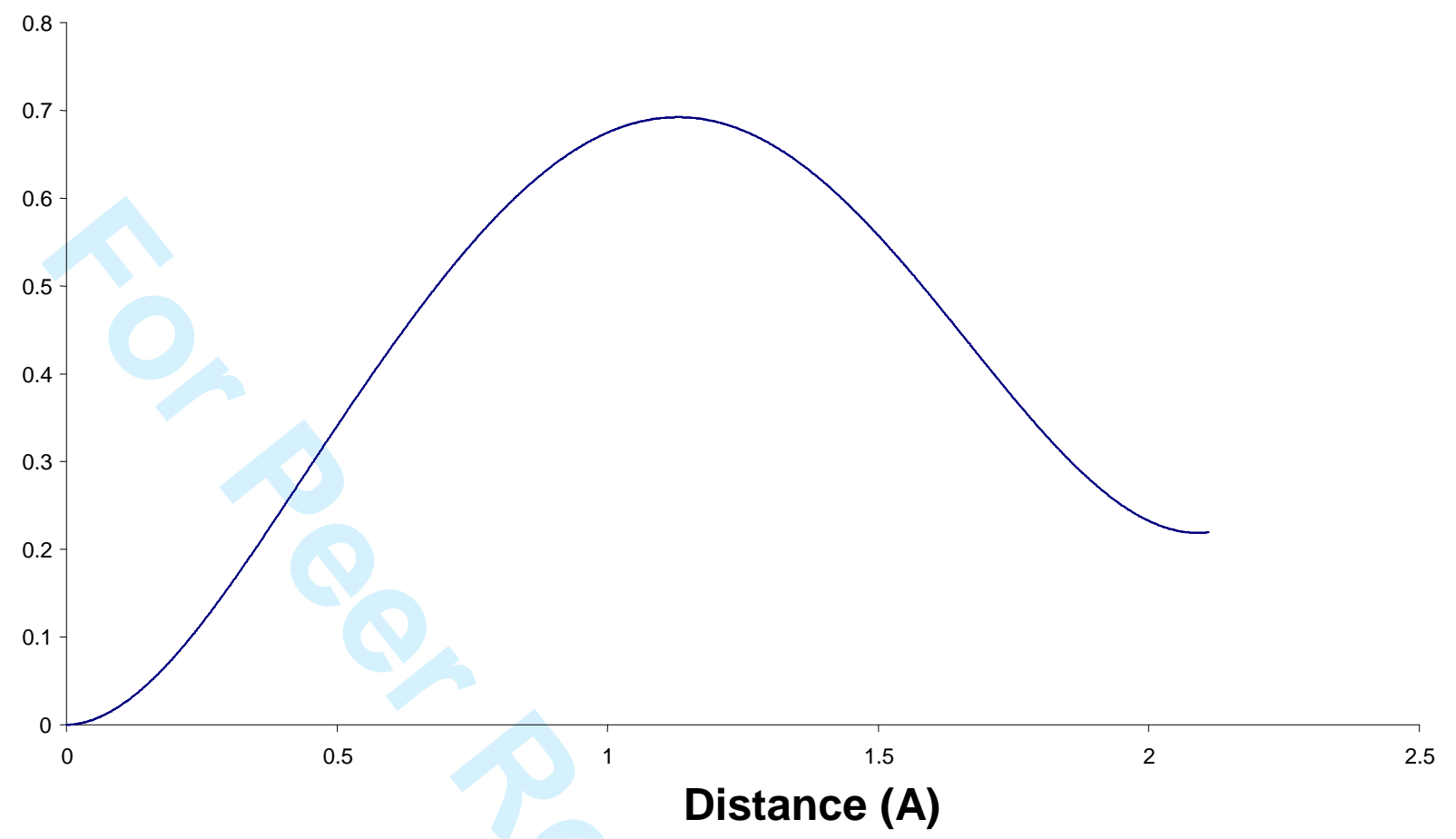

Figure 6: Energy Profile of Oxygen Migration from Bulk to Just Under the $\{111\}$ Surface

http://mc.manuscriptcentral.com/tandf/jenmol 


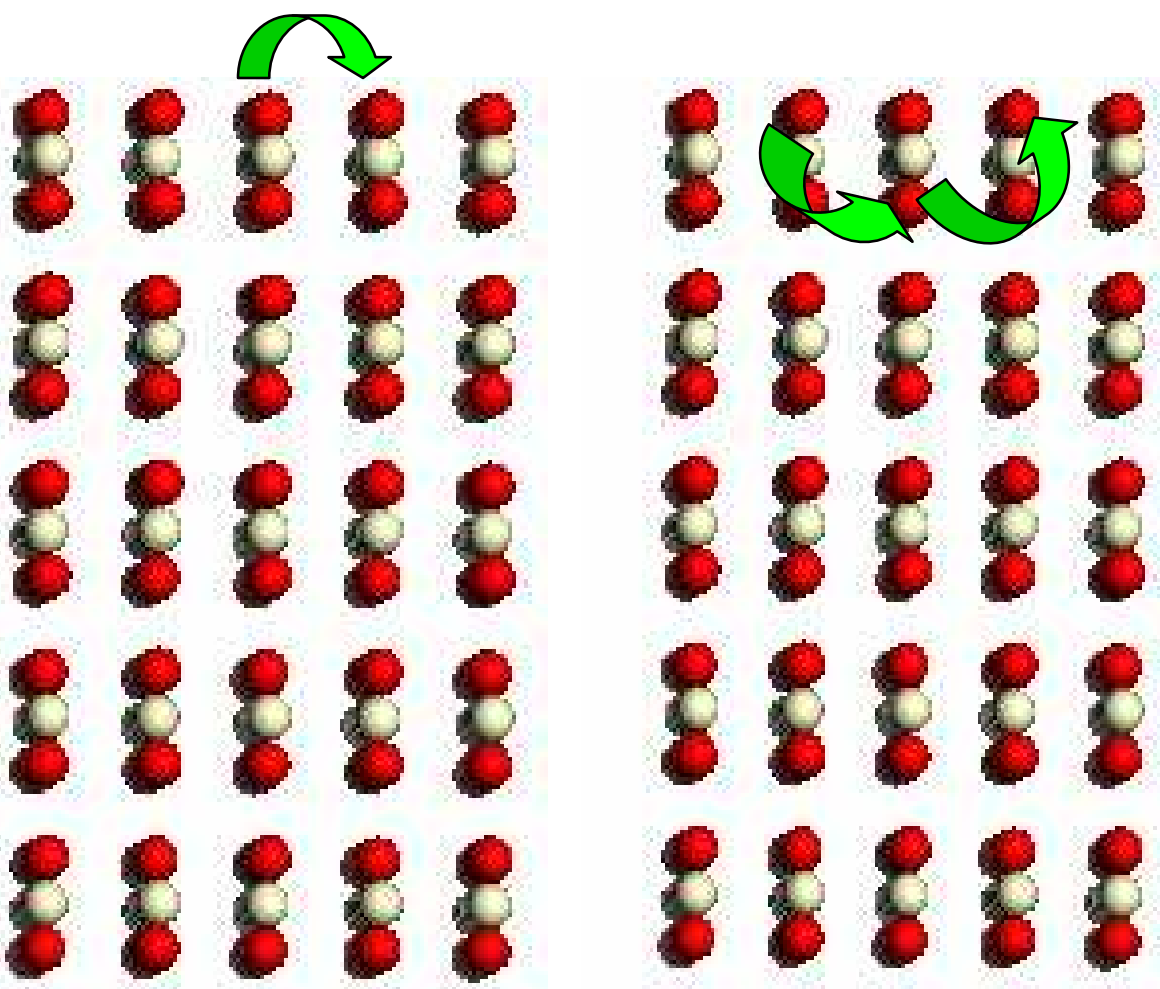

Figure 7 (a) Schematic of oxygen hopping across the surface. $\Delta \mathrm{G} \sim 2.5 \mathrm{eV}$

Figure 7 (b) Schematic of oxygen moving through the surface. $\Delta \mathrm{G} \sim 0.3 \mathrm{eV}$

@ $\mathbf{T}=\mathbf{3 0 0} \mathbf{K}$

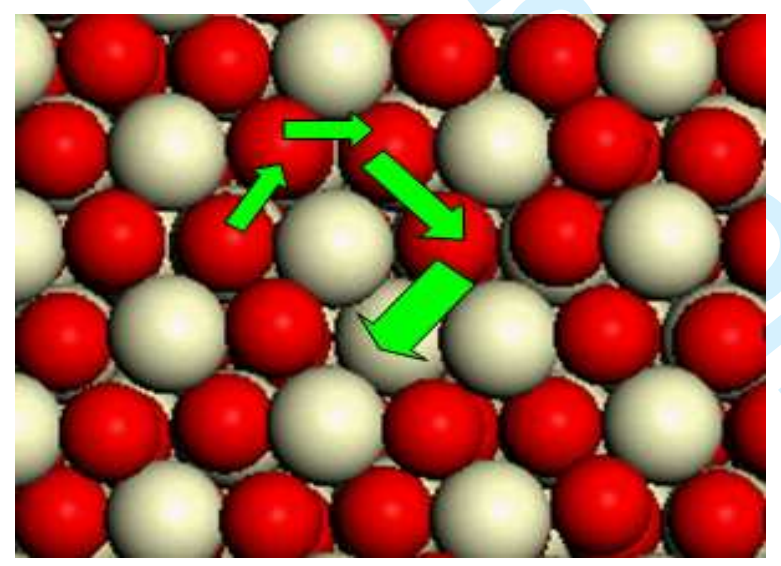

Figure 8: Pathway of Concerted Mechanism 


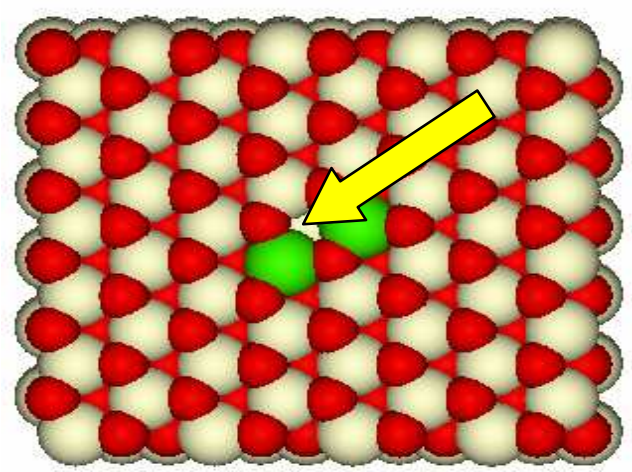

Figure 9a: Before migration and with Surface Vacancy in place. Yellow arrow points to Oxygen that is to be constrained to migrate.

Figure 9b: After Oxygen atom, originally under the surface between $\mathrm{Ce}^{3+}$ ions, has migrated to the surface leaving vacancy just

under the surface and between $\mathrm{Ce}^{3+}$ ions. Green $=\mathrm{Ce}^{3+}$,White $=\mathrm{Ce}^{4+}$, $\mathrm{Red}=\mathrm{O}^{2-}$ The resultant subsurface vacancy is highlighted by the blue arrow.

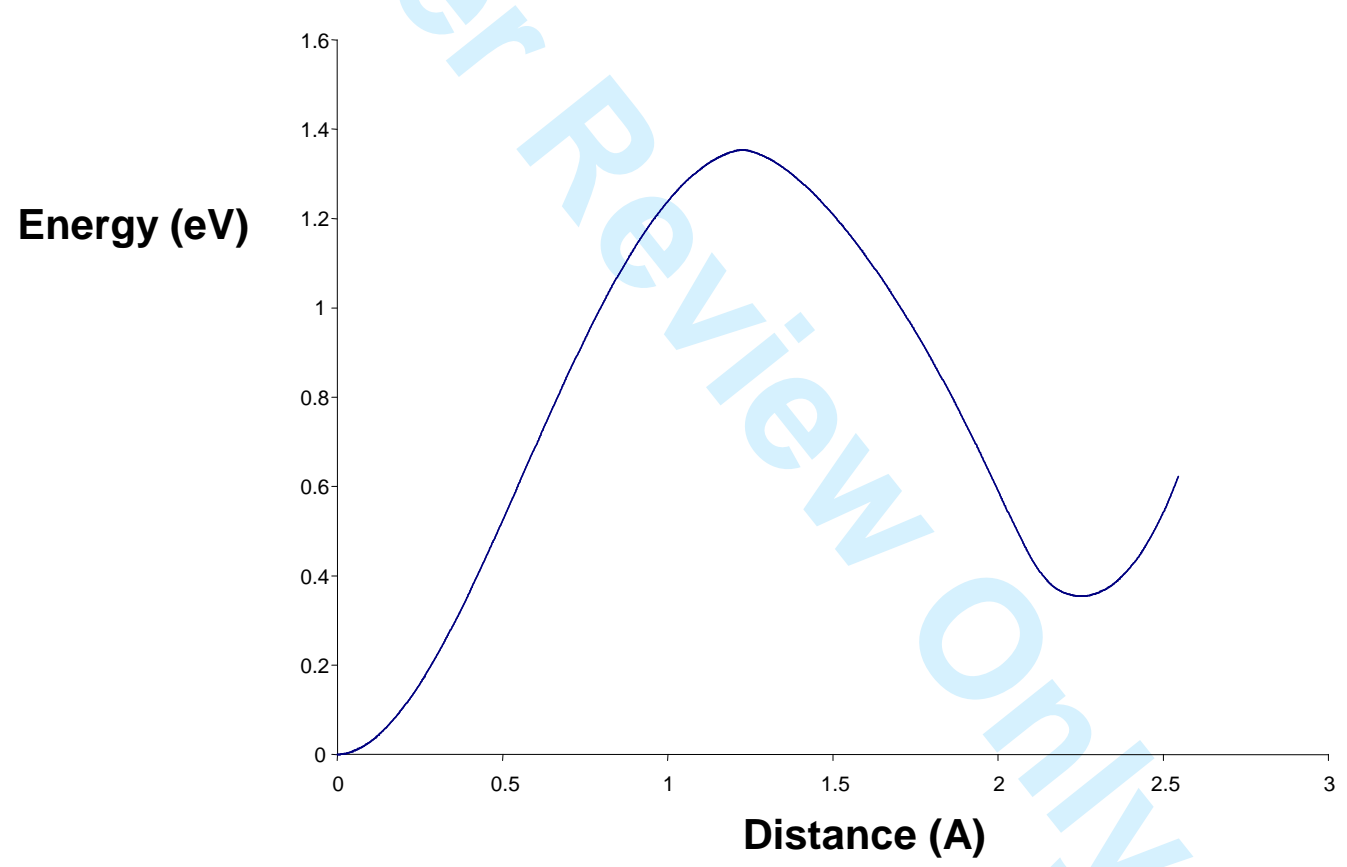

Figure 10: Energy Profile of Oxygen atom migration between $\mathrm{Ce}^{3+}$ ions onto the Surface 

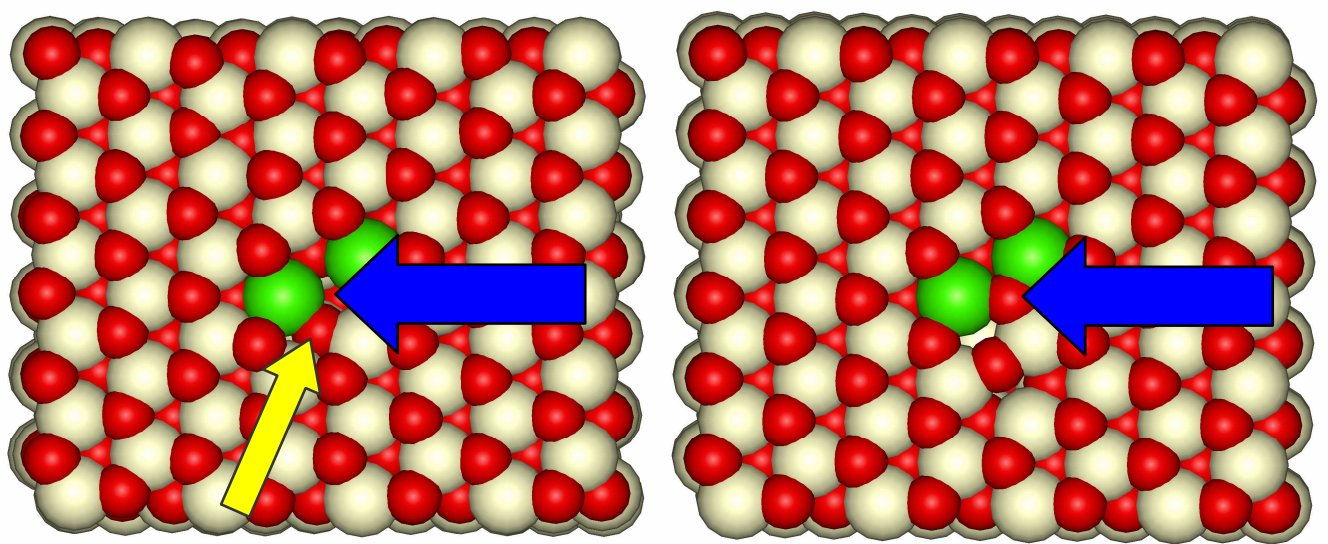

Figure 11a: Before migration and with Surface Vacancy in place (blue arrow). Yellow Arrow points to Oxygen atom to migrate from just beneath the surface.

Figure 11b: After. Oxygen atom, originally under the surface and below the vacancy, has migrated to the surface leaving vacancy just under the surface and below the left $\mathrm{Ce}^{3+}$ ion. Green $=\mathrm{Ce}^{3+}$, White $=\mathrm{Ce}^{4+}, \mathrm{Red}=\mathrm{O}^{2-}$

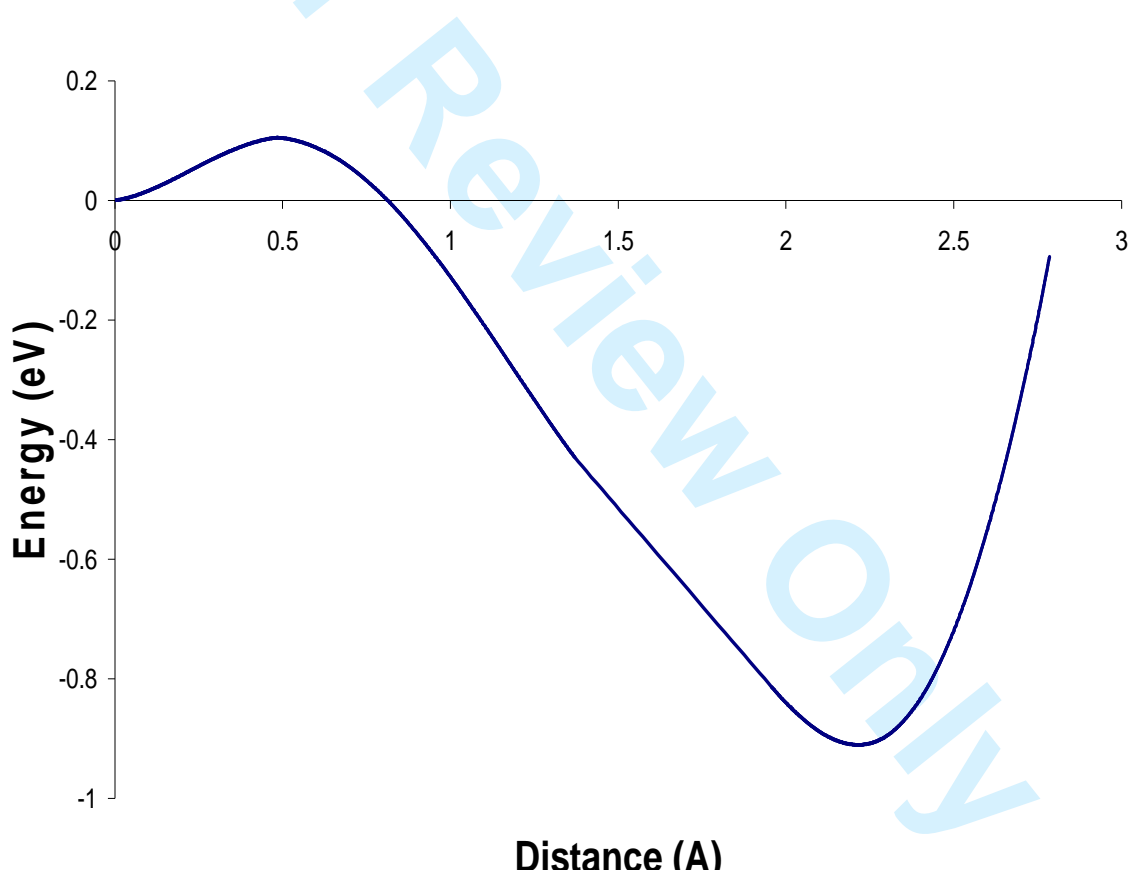

Figure 12: Energy Profile of Oxygen atom migration From under the Left $\mathrm{Hand}^{\mathrm{Ce}^{3+}}$ ion into the Surface Vacancy between Compensating $\mathrm{Ce}^{3+}$ ions 


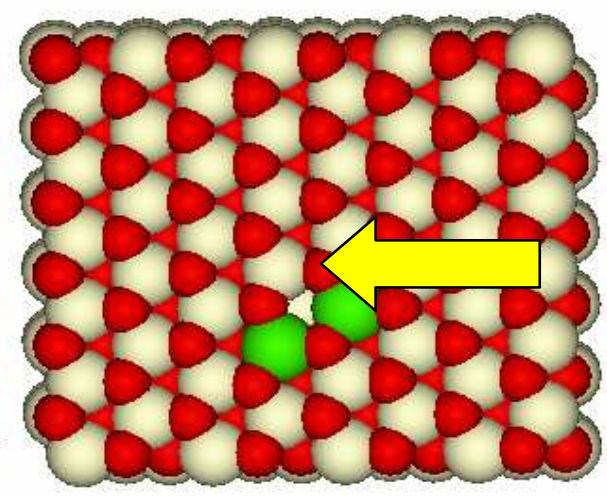

Figure 13a Before migration and with Surface Vacancy in place.

Figure 13b: After. Oxygen atom, originally under the surface between $\mathrm{Ce}^{3+}$ ions, has migrated to the surface leaving vacancy just

under the surface and between $\mathrm{Ce}^{3+}$ ions. Green $=\mathrm{Ce}^{3+}$, White $=\mathrm{Ce}^{4+}, \mathrm{Red}=\mathrm{O}^{2-}$

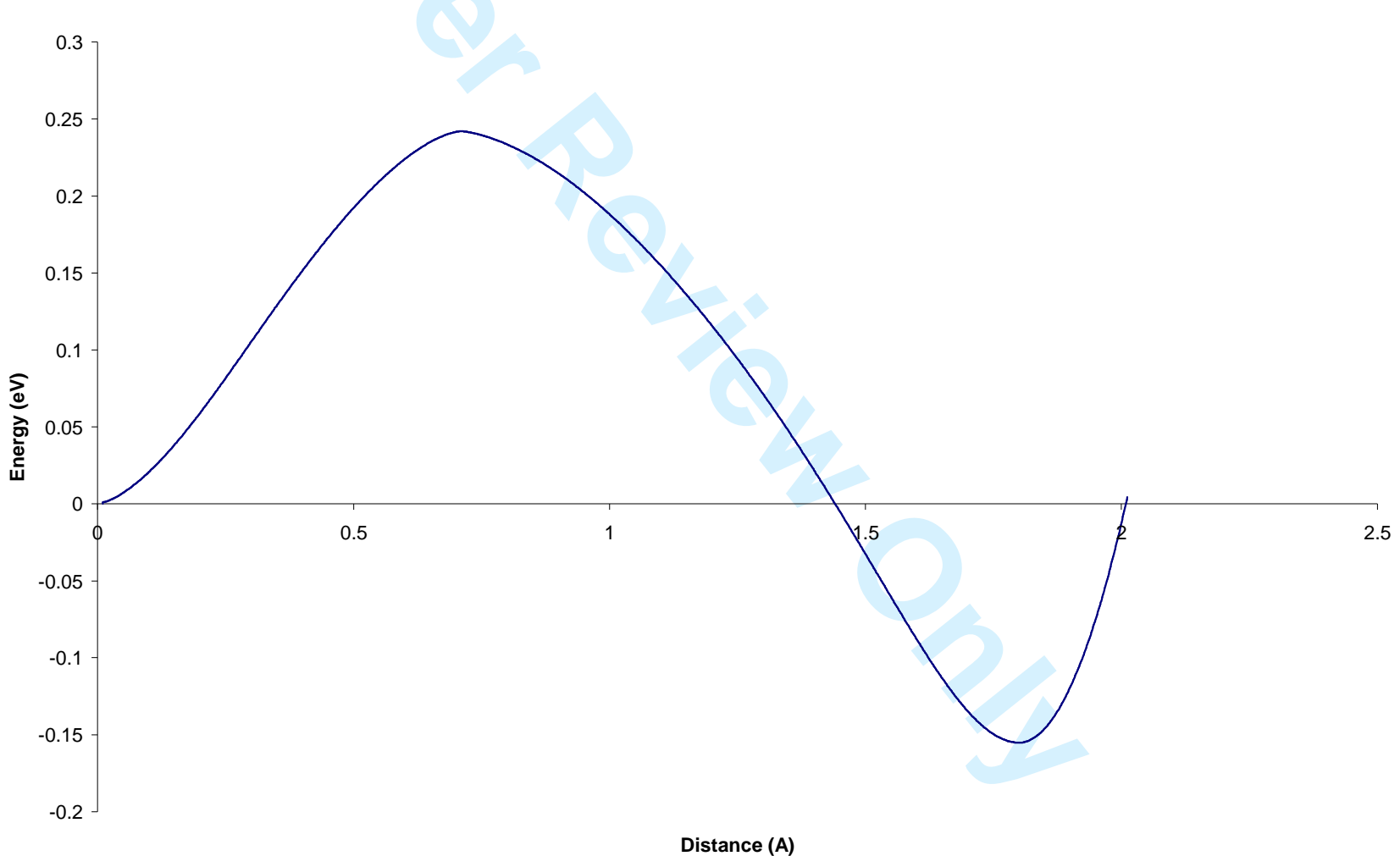

Figure 14: Energy Profile of Oxygen atom migration Away from $\mathrm{Ce}^{3+}$ ions onto the Surface 


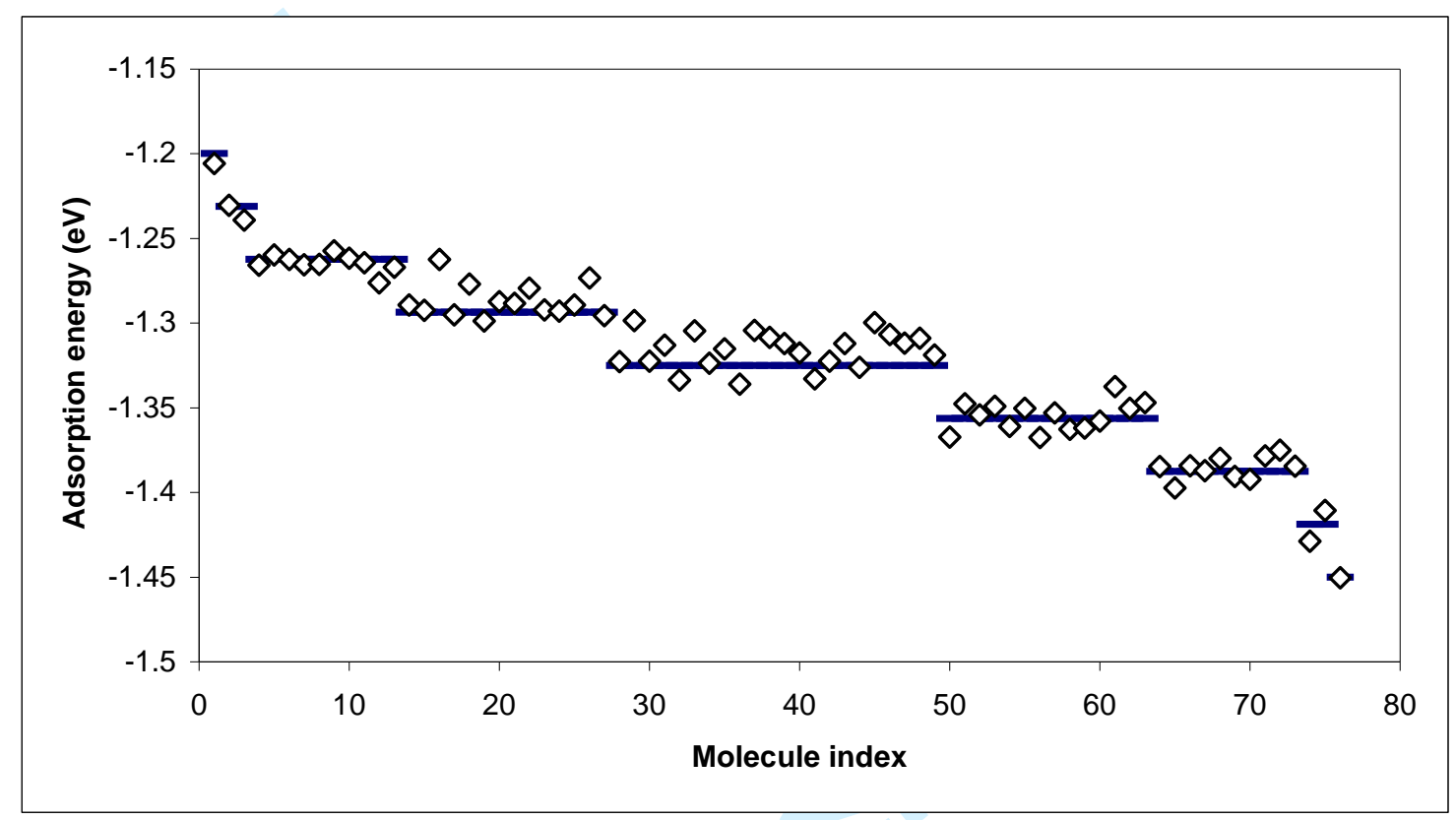

Figure 15: Adsorption energy of the 76 PCDD

http://mc.manuscriptcentral.com/tandf/jenmol 
Figure 16: Adsorption position of a PCDD congener. The surface is parallel to the page. The shaded atoms represent the lower layers. Surface: Si Large light grey, O small dark grey. Molecule: H white, Cl light grey, $\mathrm{C}$ grey, $\mathrm{O}$ dark grey 


1
2
3
4
5
6
7
8
9
10
11
12
13
14
15
16
17
18
19
20
21
22
23
24
25
26
27
28
29
30
31
32
33
34
35
36
37
38
39
40
41
42
43
44
45
46
47
48
49
50
51
52
53
54
55
56
57
58
59
60

Figure 17: Initial configuration of eight $\mathrm{MgO}$ nanoparticles before aggregation 


\section{Potential energy against time of an MD simulation of $8 \mathrm{MgO}$ nanoparticles}

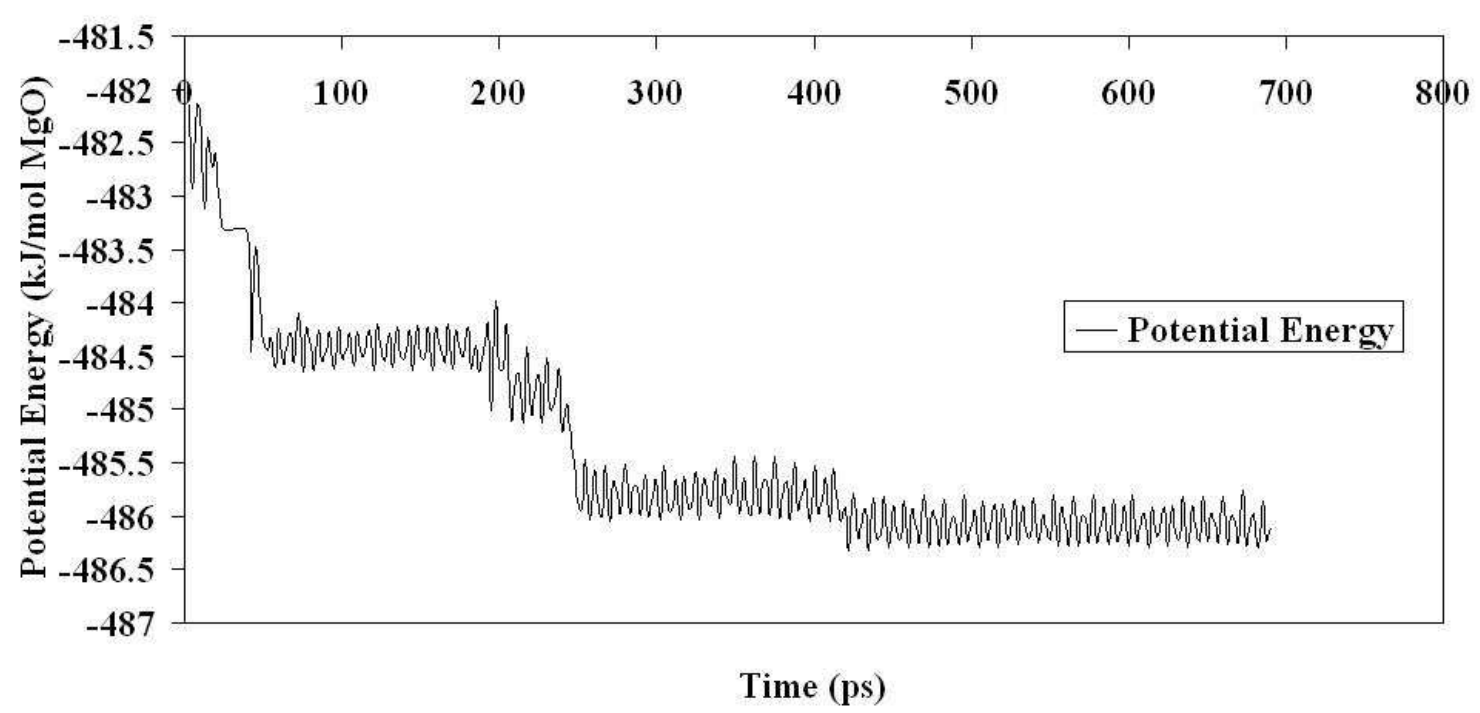

Figure 18: Potential energy per magnesium ion against time of an MD simulation of $8 \mathrm{MgO}$ nanoparticles
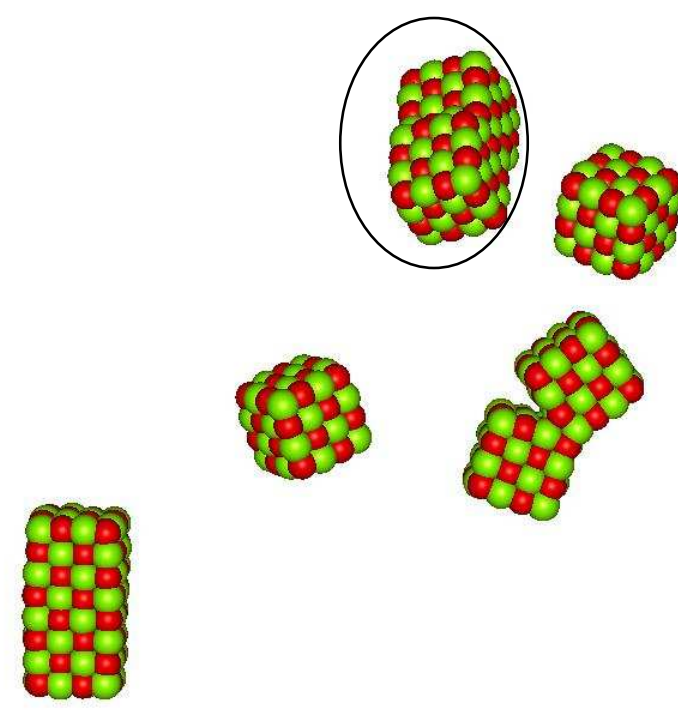

Figure 19: A snapshot of the MD simulation at 30ps for the MgO system 
Figure20: A snapshot of the MD simulation at 60ps for the MgO system
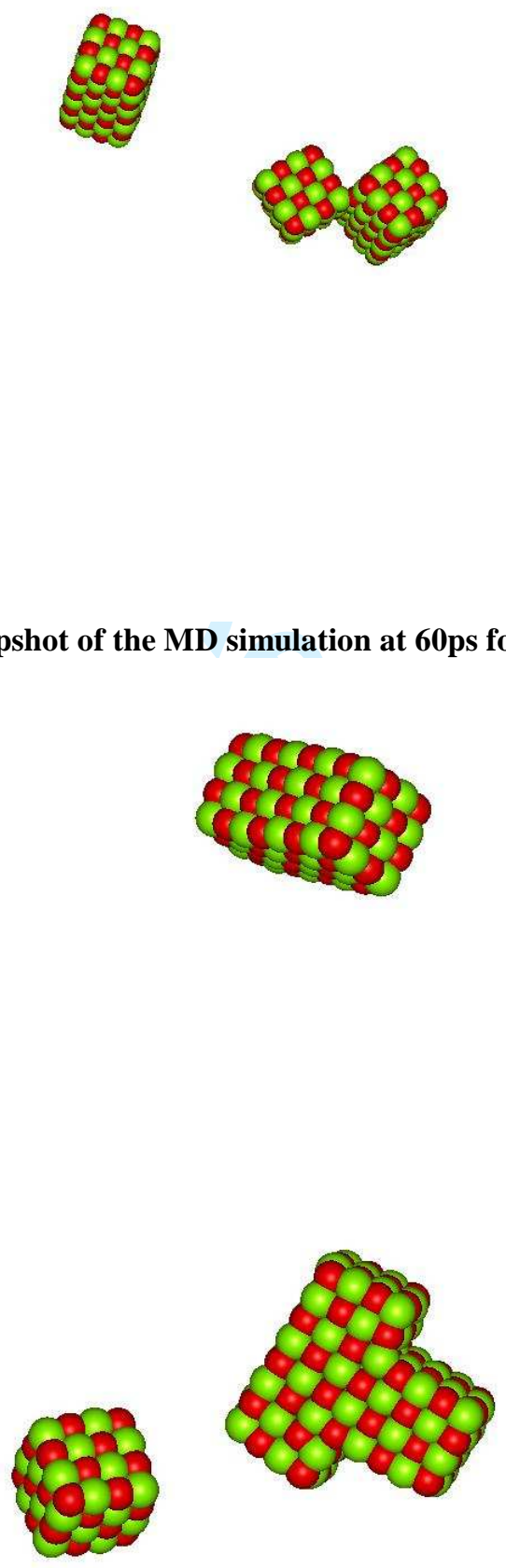

Figure 21: A snapshot of the MD simulation at 220ps for the MgO system 
Figure 22: A snapshot of the MD simulation at 275ps for the MgO system

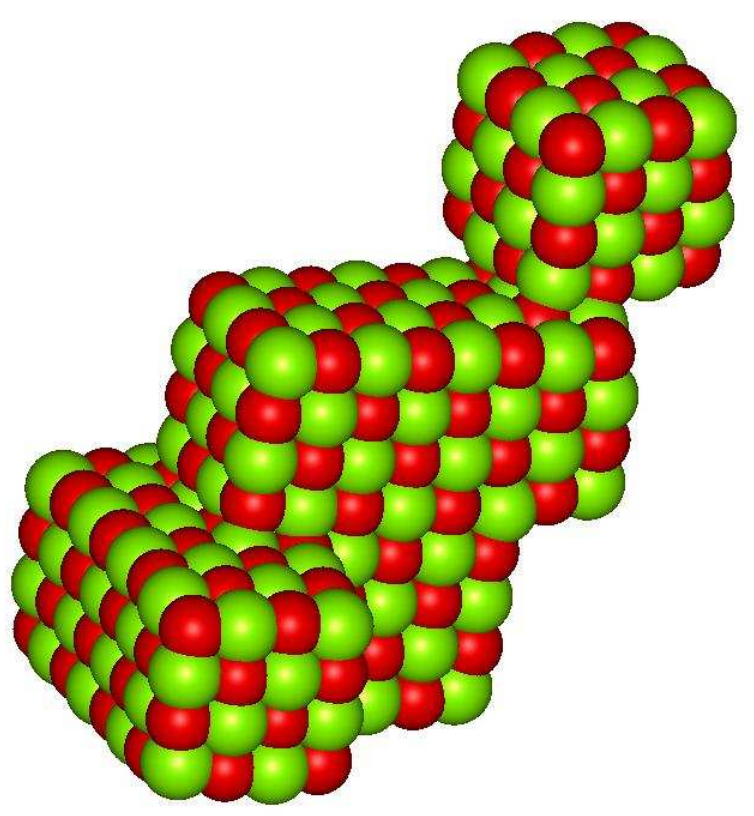

Figure 23: Final configuration of the aggregated MgO nanoparticles 


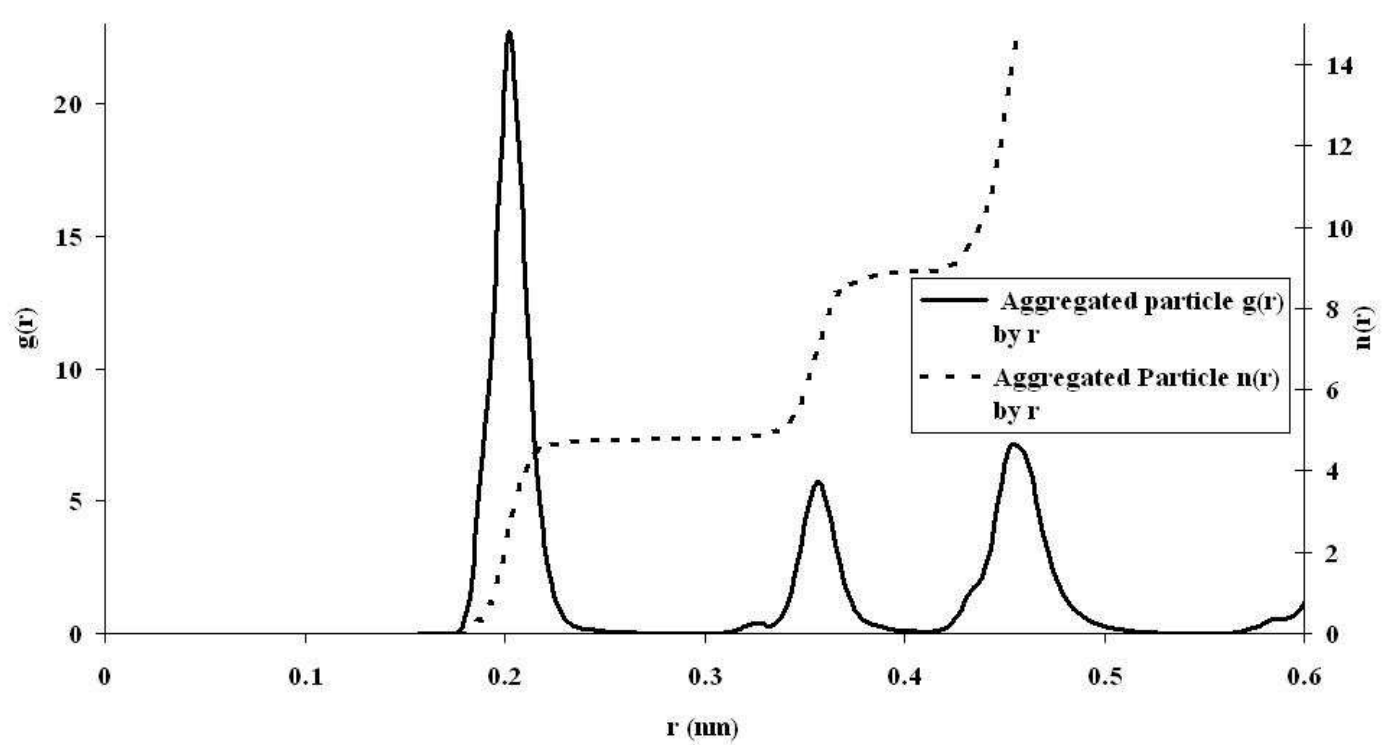

Figure 8b: Mg-O RDF of Bulk Magnesiun Oxide

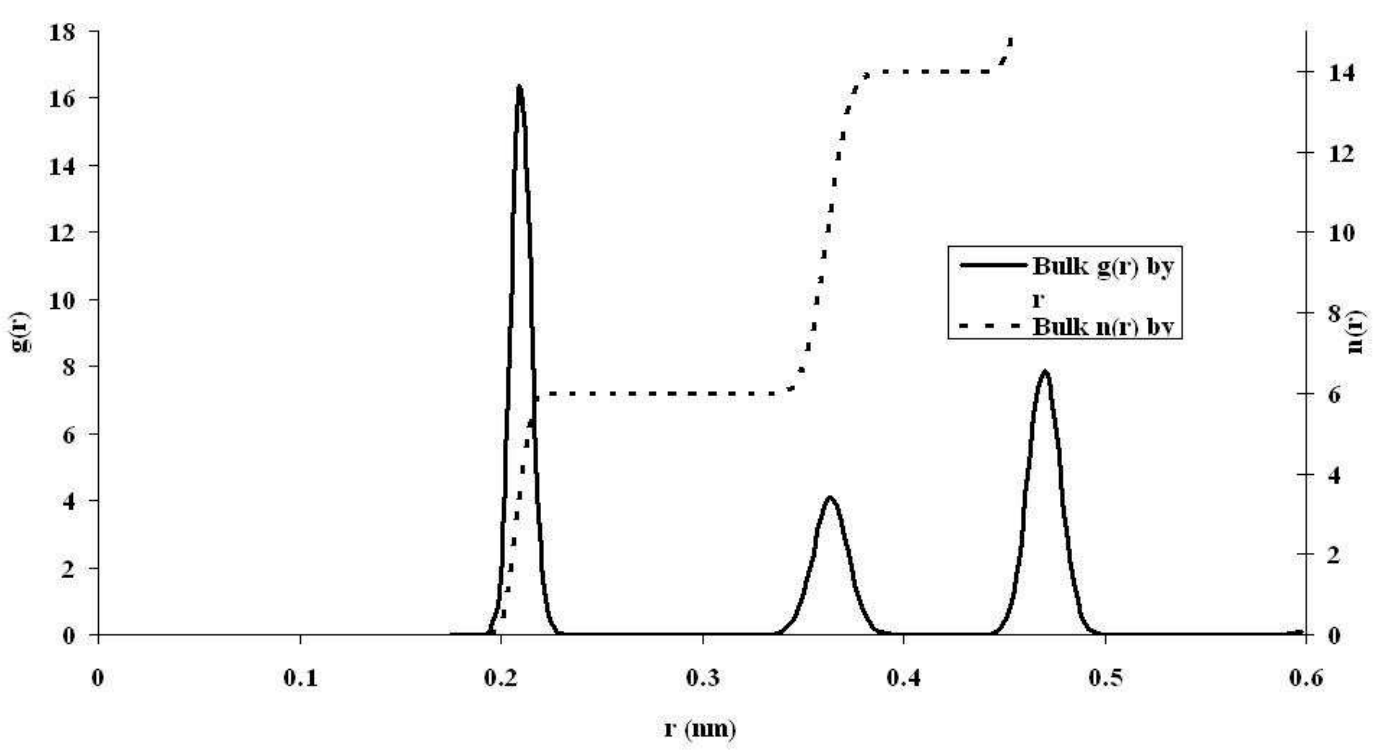

Figure 24: RDF of MgO (a) aggregated nanoparticle and (b) Bulk 
Figure 25: Initial configuration of calcite nanoparticles for MD simulation

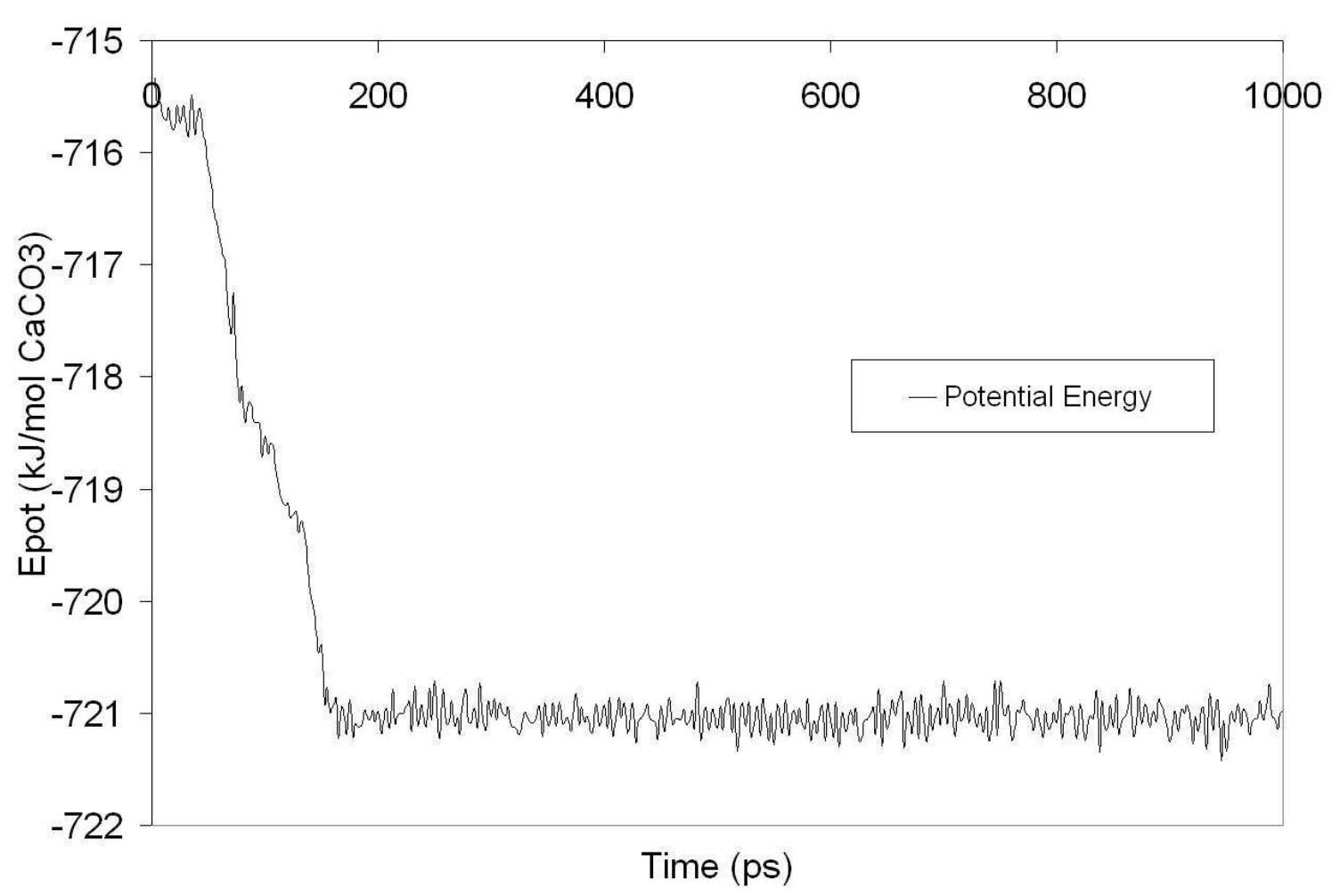

Figure 26: Potential energy per calcium ion against time of an MD simulation of 8 calcite nanoparticles 

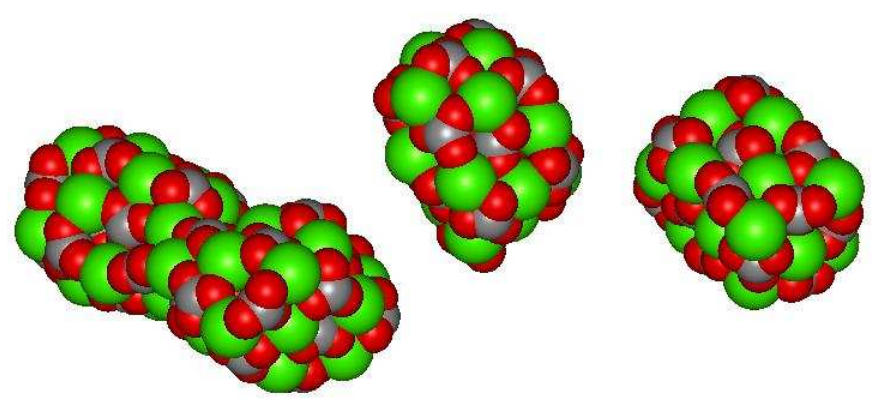

Figure 27: A snapshot of the MD simulation at 50ps for the calcite 1.6nm system 

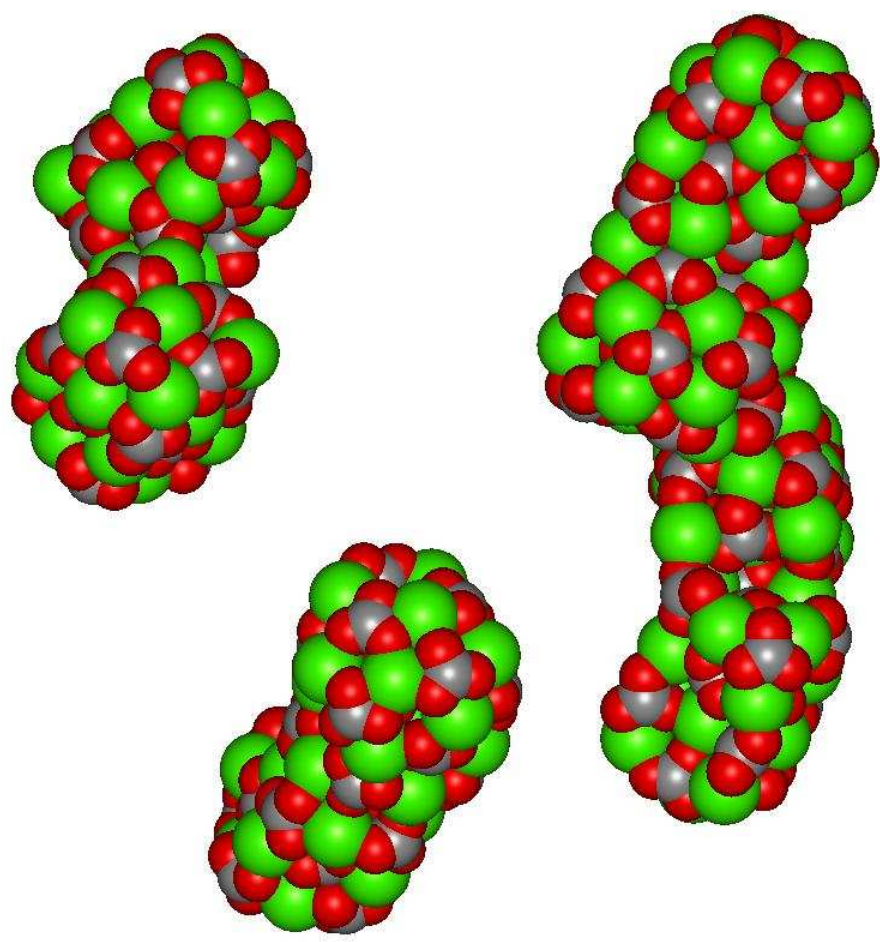

Figure 28: A snapshot of the MD simulation at $87 \mathrm{ps}$ for the calcite 1.6nm system 


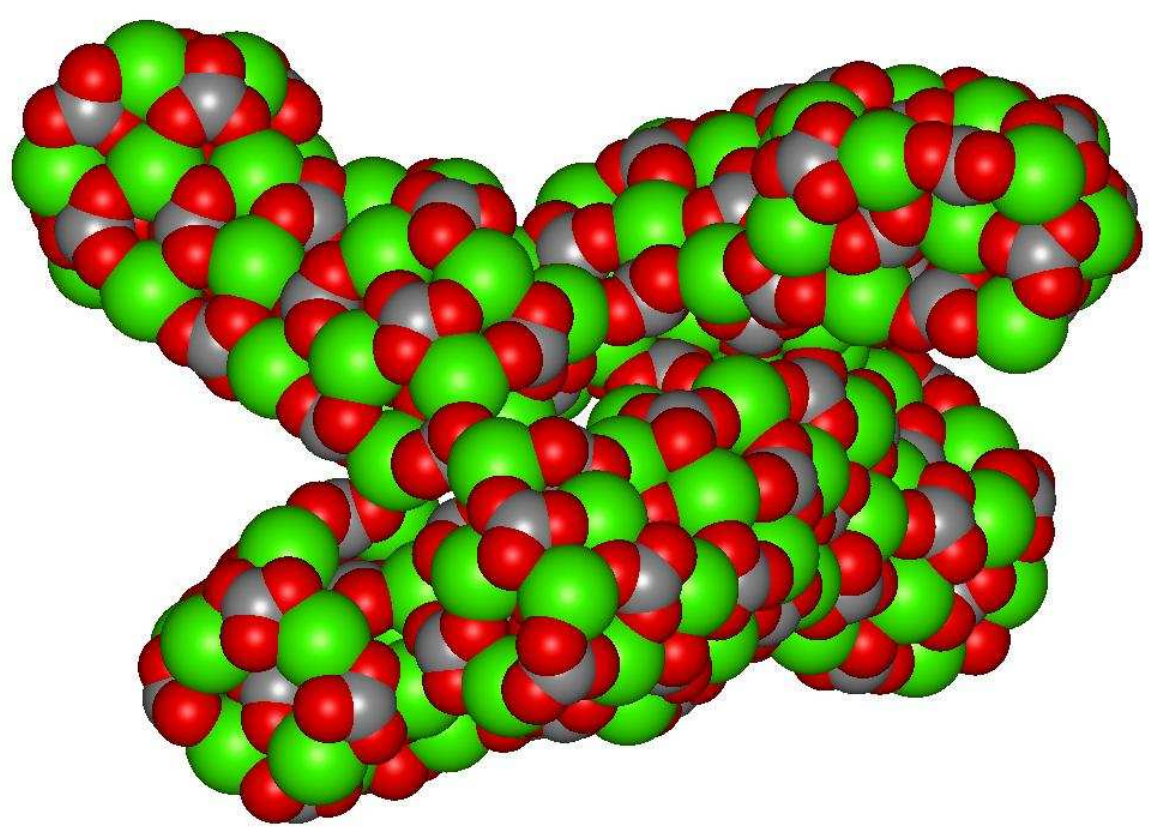

Figure 29: snapshot of the MD simulation at 140ps for the calcite 1.6nm system

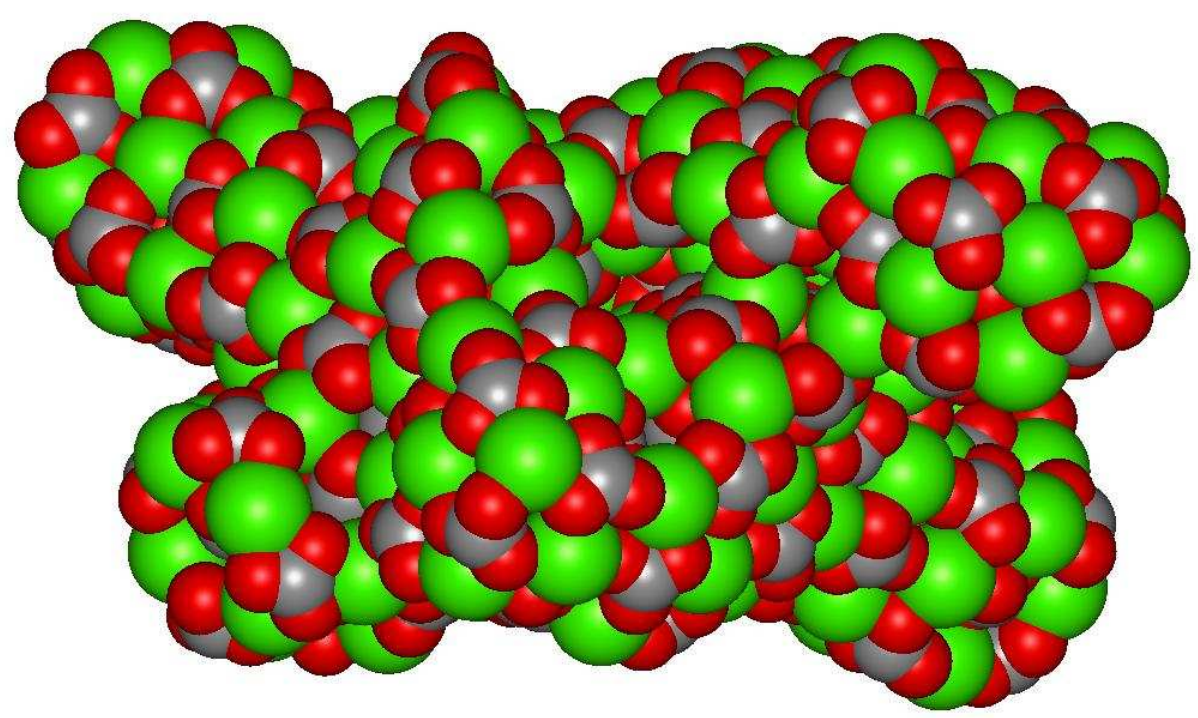

Figure 30: Final configuration of the aggregated calcite nanoparticles 


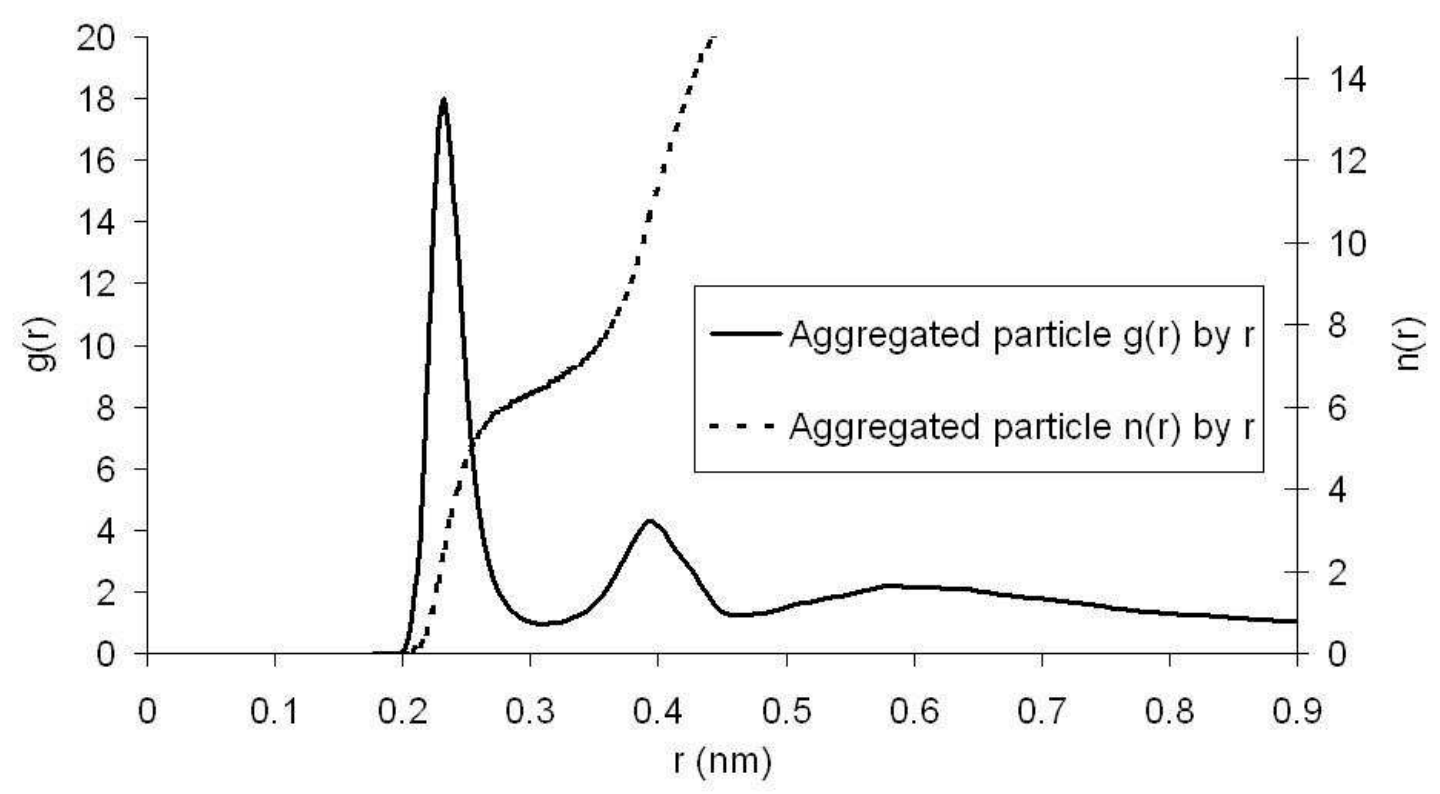

Figure 15b: Ca-O RDF in Bulk Calcite

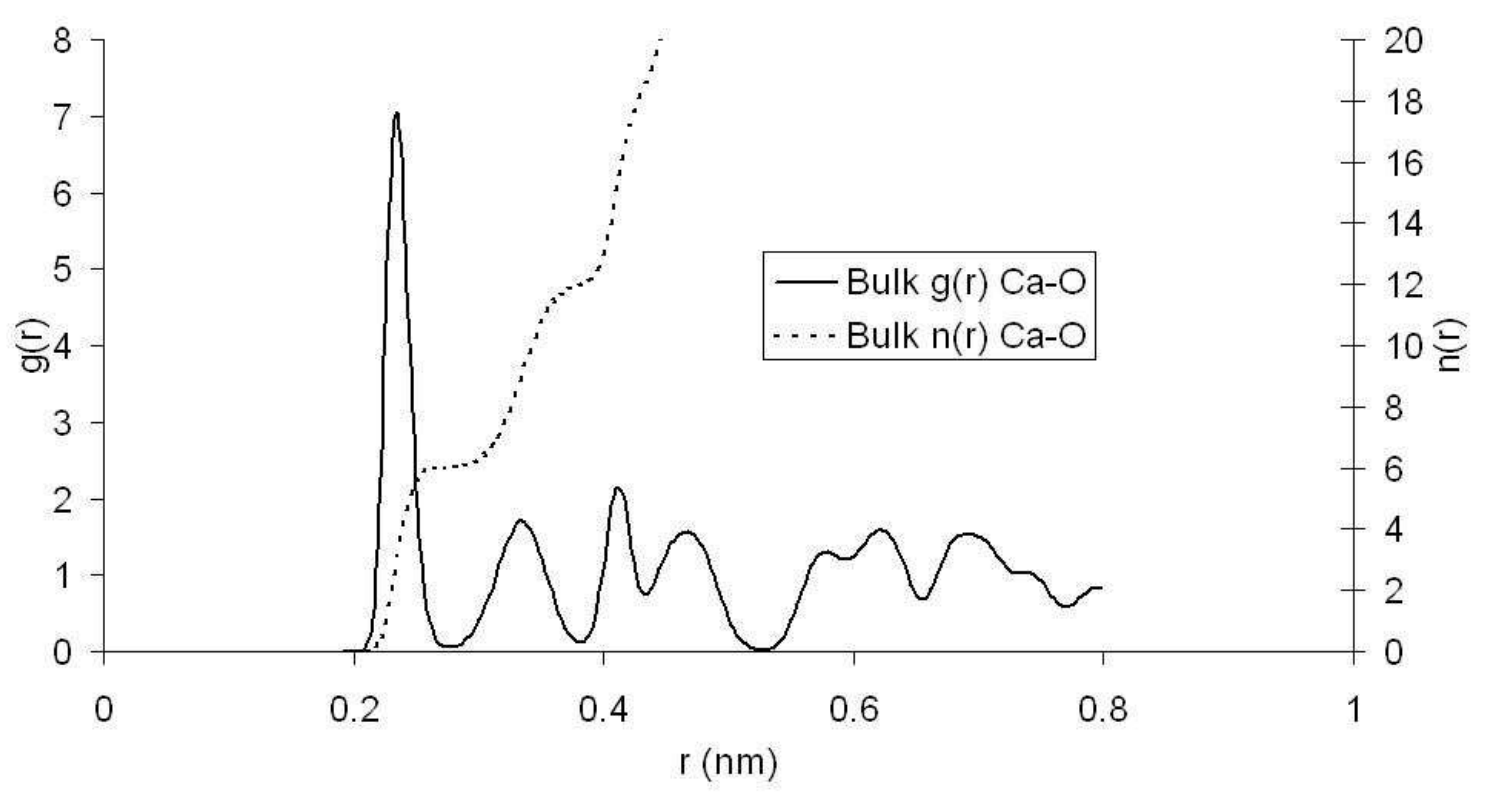

Figure 31: RDF of the (a) aggregated calcite nanoparticles and (b) bulk calcite 


\begin{tabular}{lc}
\hline \hline \multicolumn{1}{c}{ Specie } & Charge \\
\hline $\mathrm{H}$ & .20 \\
$\mathrm{Cl}$ & -.03 \\
$\mathrm{O}$ & -.50 \\
$\mathrm{C}(\mathrm{H}$ bonded $)$ & -.20 \\
$\mathrm{C}(\mathrm{Cl}$ bonded $)$ & .03 \\
$\mathrm{C}(\mathrm{O}$ bonded $)$ & .25 \\
\hline \hline
\end{tabular}

Table 1: Charge model (in e) 